\title{
QUANTIFYING RADIOBIOLOGICAL VARIATION IN CANCER RADIOTHERAPY USING MONTE CARLO SIMULATION AND DOPED PLASTIC SCINTILLATORS
}

\author{
by
}

Humza Nusrat

B.Sc. (Hons), University of Ontario Institute of Technology, Oshawa, Canada, 2013

\author{
A dissertation \\ presented to Ryerson University \\ in partial fulfillment of the \\ requirements for the degree of \\ Doctor of Philosophy \\ in the program of \\ Biomedical Physics
}

Toronto, Ontario, Canada, 2019

(C) Humza Nusrat, 2019 


\section{Author's Declaration}

I hereby declare that I am the sole author of this dissertation. This is a true copy of the dissertation, including any required final revisions, as accepted by my examiners.

I authorize Ryerson University to lend this dissertation to other institutions or individuals for the purpose of their scholarly research.

I further authorize Ryerson University to reproduce this dissertation by photocopying or by other means, in total or in part, at the request of other institutions or individuals for the purpose of their scholarly work.

I understand that my dissertation may be made electronically available to the public. 


\title{
Abstract
}

\section{Quantifying Radiobiological Variation in Cancer Radiotherapy using Monte Carlo Simulation and Doped Plastic Scintillators}

\author{
Humza Nusrat \\ Doctor of Philosophy, Biomedical Physics (CAMPEP)
}

Ryerson University, 2019

This dissertation examines the extent to which radiobiological variations occur in photon radiotherapy, and then presents a novel methodology and detector prototype to measure this variation.

In the first section, I examine the change in maximum $\mathrm{RBE}\left(\mathrm{RBE}_{\mathrm{M}}\right)$ outside the primary field in open and composite $6 \mathrm{MV}$ x-ray beams. This is done using Monte Carlo simulation and microdosimetric techniques. It was found that when comparing an open $10 \times 10 \mathrm{~cm}^{2} 6 \mathrm{MV}$ beam to a composite $10 \times 10 \mathrm{~cm}^{2}$ beam comprising one hundred $1 \times 1$ $\mathrm{cm}^{2}$ beamlets, the out-of-field increase in RBE occurs much closer to the field edge in the composite case. This finding may have consequences for IMRT cases in which large amount of scattered radiation may be causing a higher than expected effective dose to organs at risk.

In the second section, the maximum RBE variation is examined in the context of brachytherapy. The sources examined include ${ }^{192} \mathrm{Ir},{ }^{125} \mathrm{I}$, and ${ }^{169} \mathrm{Yb}$. It was determined that maximum RBE of ${ }^{125}$ I relative to the source position did not vary significantly as distance from the source was increased, however, ${ }^{192} \mathrm{Ir}$ and ${ }^{169} \mathrm{Yb}$ were found to exhibit 
$\mathrm{RBE}_{\mathrm{M}}$ increases of $3.0 \%$ and $6.6 \%$ at a distance of $8 \mathrm{~cm}$, respectively. Also, the impact of this variation on an HDR ${ }^{192}$ Ir prostate treatment plan was examined; it was found that $\mathrm{RBE}_{\mathrm{M}}$ hotspots of $+3.6 \%$ occur at the treatment plan's periphery.

In the third part, the impact of lead doping on plastic scintillator response is quantified, a major step required for the development of the LET detector prototype. In this stage, 4 differently doped plastic scintillators were obtained, and measurements were conducted in low and medium LET beams. Using Geant4 Monte Carlo and the measured scintillator responses, the scintillator parameters: $k B$ and $L_{0}$ were determined as a function of dopant concentration and effective atomic number.

Finally, the uniquely energy dependent scintillators were combined into a detector prototype used to measure the LET spectra produced by five low energy photon beams. These beams included four orthovoltage energies $(100,180,250$, and $300 \mathrm{kVp}$ ) along with an ${ }^{192} \mathrm{Ir}$ HDR source. In this proof-of-principle work, the detector prototype and technique was found to accurately determine the LET spectra and the mean LET for all beams with the exception of the $100 \mathrm{kVp}$ orthovoltage beam.

Potential applications for the real-time LET detector prototype and technique described in this dissertation include LET measurement in radiotherapy, allowing for biologically optimized treatment plans improving patient care. This technique and prototype also has numerous applications in non-medical fields such as health physics, space travel dosimetry, and nuclear safety. 


\section{Acknowledgements}

First and foremost, I must give all praise and thanks to God Almighty for allowing me to learn, grow, and thrive as a graduate student. The past five years at Ryerson University have been the best years of my life, and it is a bitter sweet feeling to realize that I will soon have to say goodbye.

I express my deepest gratitude to my supervisor, Dr. Arman Sarfehnia. I have been extremely lucky to have you as my mentor. Thank you for seeing potential in me when I interviewed at Ryerson all of those years ago; it turned out to be a turning point in my life. Over the years, you have treated me with kindness, patience, wisdom, and care. Your mentorship and guidance have allowed me to think outside the box and enter the field that I love. I hope to one day follow in your footsteps and have a research lab of my own, and I will do my best to treat my students with the same attention and care you've given me. Thank you for everything, Arman. I will always be indebted to you for taking a chance on me and letting me learn at your side.

A very sincere thank you to Dr. Geordi Pang. Despite being one of the busiest physicists due to your additional radiation safety duties, you were always there for me whenever I became stuck. Without your wisdom and guidance, this project would not have moved forward. Thank you for always treating me with kindness and patience.

My sincere thanks to my committee members Dr. Carl Kumaradas and Dr. Ana Pejovic-Milic. I will forever be grateful for your guidance and mentorship, and for welcoming me into the Ryerson Biomedical Physics family. 
My sincere gratitude to Dr. James Grafe for your mentorship and guidance. Working as your TA for several courses allowed me to pick up your teaching skills and habits - someday, I hope to positively impact students through the classroom the way you do.

Thank you to Dr. Syed Bilal Ahmad for teaching me how to use Geant4 and for helping me with my countless questions. Your kindness and compassion will never be forgotten.

Thank you to all of my colleagues and friends over the years for inspiring me and pushing me to greater heights. Your friendship and collegiality have formed who I am today. Thank you to Alex, Danny, Jae, Dan, Martin, Mark, and many others - there are simply too many of you to name. At Sunnybrook, my sincere thank you to Philip, Karl, lan, Harry, Mark, and Bilal for all of the great conversations and collaborations.

To my dear brothers: Waleed, Omar, and Rahim. Thank you for always having my back and being there for me. Your encouragement and words of wisdom have helped me tremendously over the duration of this degree. Thank you to my grandparents - Ammi and Baba, thank you for loving me and being proud of me. To my other grandparents - Nana and Nanno, thank you for always being excited about my work and giving me your love. Thank you to all of my family members - my aunts, uncles, and cousins - for supporting me and believing in me.

To my dear Sameeha, I can never express how much your unconditional love and support mean to me. Thank you for always being there for me. 
To my dear Mom and Dad, thank you for always pushing me to pursue my goals and for supporting me whenever I was going through tough times. It is only through your love that I have been able to make it through to the other side of this degree. If it wasn't for your guidance and inspiration, I never would have embarked on this journey. You both inspire me more than I can express in words. That is why, it is to you, my dearest Mother and Father, I dedicate this dissertation. 


\section{Table of Contents}

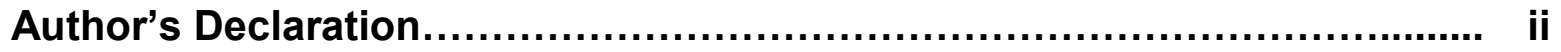

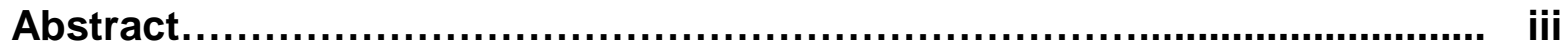

Acknowledgements.................................................................... v

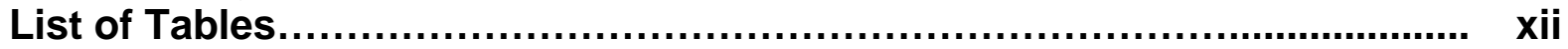

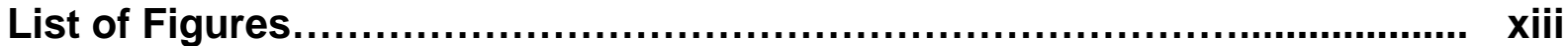

1. Introduction............................................................................ 1

1.1. Cancer..................................................................... 1

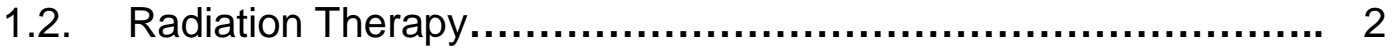

1.3. Rationale and Significance........................................ 11

1.4. Specific Aims.......................................................... 13

1.5. Thesis Organization................................................. 14

2. Radiation Dosimetry....................................................... 15

2.1. Electromagnetic Radiation........................................ 16

2.2. Charged Particle Interactions.......................................... 19

2.3. Stopping Power and Linear Energy Transfer....................... 21

2.4. Absorbed Dose....................................................... 23

2.5. Relative Biological Effectiveness................................... 24

2.6. Monte Carlo Simulation............................................... 28

2.7. Plastic Scintillators............................................ 32

3. Evaluating the Biological Impact of Increased Scattered Radiation in Single and Composite Field Radiation Beams.............................. 36

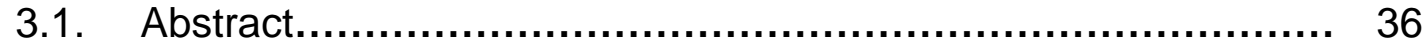

3.2. Introduction.................................................... 37

3.3. Materials and Methods.............................................. 41

3.3.1. $\alpha / \beta$ Ratio Formalism.......................................... 41

3.3.2. Obtaining $n(E)$, the Electron Energy Spectra................ 42

3.3.3. Composite $10 \times 10 \mathrm{~cm}^{2}$ Field................................ 44

3.4. Results............................................................... 44

3.5. Discussion............................................................ 47

3.6. Conclusions...................................................... 52

3.7. Acknowledgements.................................................. 53

4. Maximum RBE Change in 192Ir, $125 \mathrm{I}$, and $169 \mathrm{Yb}$ Brachytherapy and the Corresponding Effect on Treatment Planning........................... 54

4.1. Abstract................................................................ 54

4.2. Introduction.......................................................... 55

4.3. Materials and Methods............................................ 61

4.3.1. Radioactive Sources....................................... 62 


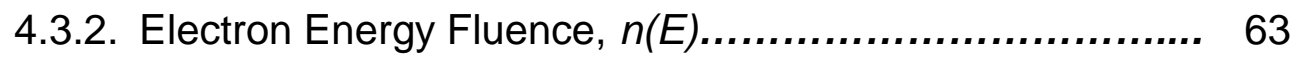

4.3.3. Calculating Relative Biological Effectiveness (RBE)........ 66

4.3.4. $\mathrm{RBE}_{\mathrm{M}}$ Distribution of a Typical Prostate Brachytherapy Treatment....................................................... 67

4.4. Results............................................................ 68

4.5. Discussion...................................................... 73

4.6. Conclusions....................................................... 78

4.7. Acknowledgements.............................................. 78

5. Quantifying the Impact of Lead Doping on Plastic Scintillator

Response to Radiation........................................................... 79

5.1. Abstract....................................................... 79

5.2. Introduction.................................................... 80

5.3. Materials and Methods........................................... 84

5.3.1. Overall Methodology........................................ 84

5.3.2. Measurements................................................ 85

5.3.3. Monte Carlo Simulation....................................... 88

5.3.4. Scintillator Response Analysis............................. 89

5.4. Results............................................................... 90

5.4.1. Lead Doping and Measured Response...................... 90

5.4.2. Birks' Law Parameter Optimization........................... 94

5.5. Discussion............................................................... 98

5.6. Conclusions.................................................... 102

5.7. Acknowledgements................................................. 102

6. Novel Method for LET Measurement in Low Energy Photon Beams using Doped Plastic Scintillators: Proof-of-Principle........................ 104

6.1. Abstract............................................................. 104

6.2. Introduction...................................................... 105

6.3. Materials and Methods............................................. 108

6.3.1. General Formalism......................................... 108

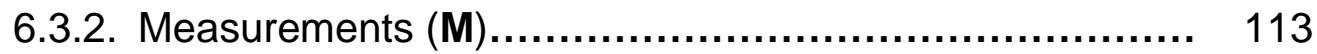

6.3.3. Response Matrix (R) ....................................... 116

6.3.4. Monte Carlo Simulation...................................... 118

6.4. Results............................................................ 120

6.5. Discussion...................................................... 126

6.6. Conclusions............................................................ 129

6.7. Acknowledgements................................................ 130

7. Conclusions and Future Work................................................. 131

7.1. Summary of Findings............................................... 131

7.1.1. Biological Impact of Scattered Radiation in a 6 MV x-ray beam. 
7.1.2. Maximum RBE Variation in Brachytherapy................ 132

7.1.3. Lead-doped Plastic Scintillators............................... 133

7.1.4. LET Measurement............................................ 133

7.2. Future Work.......................................................... 134

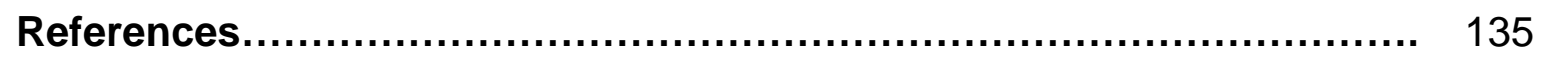

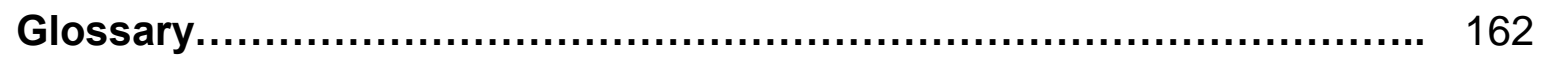




\section{List of Tables}

\begin{tabular}{|c|c|c|}
\hline $\begin{array}{c}\text { Table } \\
\#\end{array}$ & Title & $\begin{array}{c}\text { Page } \\
\#\end{array}$ \\
\hline 4.1 & $\begin{array}{l}\text { Mean energies of various radioactive sources commonly used in } \\
\text { brachytherapy. }\end{array}$ & 56 \\
\hline 4.2 & $\begin{array}{l}\text { The summary of previously determined RBE values for }{ }^{125} \mathrm{I},{ }^{192} \mathrm{Ir} \text {, and } \\
{ }^{169} \mathrm{Yb} \text { is shown below. }\end{array}$ & 60 \\
\hline 4.3 & $\begin{array}{l}\text { Summary of Monte Carlo parameters used to create the simulation for } \\
\text { obtaining RBE values of }{ }^{125} \mathrm{I},{ }^{192} \mathrm{Ir} \text {, and }{ }^{169} \mathrm{Yb} \text {. This table is based on } \\
\text { AAPM TG268 Report and 'TG268 Item \#' refers to the required Monte } \\
\text { Carlo item specified in Sechopolous et al. }\end{array}$ & 64 \\
\hline 5.1 & $\begin{array}{l}\text { Measurement Uncertainty Analysis. In the 'Output Measurement' section } \\
\text { of the uncertainty analysis, '*' is used to reference the TG- } 51 \text { protocol, } \\
\text { while '**' is used to reference the TG- } 61 \text { protocol. All uncertainty } \\
\text { components given as relative standard deviations }(k=1) \text {. }\end{array}$ & 92 \\
\hline 6.1 & Typical LET values for different types of radiation & 107 \\
\hline 6.2 & $\begin{array}{l}\text { Summary of Monte Carlo parameters used. This table is based on AAPM } \\
\text { TG268 Report and 'TG268 Item \#' refers to the required Monte Carlo } \\
\text { item specified in Sechopolous et al. }\end{array}$ & 119 \\
\hline 6.3 & $\begin{array}{l}\text { Average LET values calculated using the method described compared to } \\
\text { the average LET generated using the Monte Carlo data at a depth of } 1.5 \\
\mathrm{~cm} \text { in water. }\end{array}$ & 124 \\
\hline 6.4 & $\begin{array}{l}\text { Uncertainty Analysis for measurement and response matrices. All } \\
\text { uncertainty components given as relative standard deviations }(k=1) \text {. }\end{array}$ & 125 \\
\hline
\end{tabular}




\section{List of Figures}

\begin{tabular}{|c|c|c|}
\hline $\begin{array}{l}\text { Figure } \\
\#\end{array}$ & Caption & $\begin{array}{l}\text { Page } \\
\#\end{array}$ \\
\hline 1.1 & $\begin{array}{l}\text { Typical photo of a linear accelerator used to deliver high energy photon } \\
\text { radiotherapy to cancer patients }\end{array}$ & 5 \\
\hline 1.2 & $\begin{array}{l}\text { The MR-linac produced by Elekta, housed at Sunnybrook Health } \\
\text { Sciences Centre }\end{array}$ & 6 \\
\hline 1.3 & Decay scheme of cobalt-60 [WikiCommons Image] & 7 \\
\hline 1.4 & Leksell GammaKnife produced by Elekta & 8 \\
\hline 1.5 & $\begin{array}{l}\text { Depth vs dose distribution for a proton travelling through water. The } \\
\text { proton deposits the largest amount of energy at the end of its track } \\
\text { (Bragg Peak) }\end{array}$ & 9 \\
\hline 1.6 & $\begin{array}{l}\text { HDR brachytherapy after-loader. The high activity source is housed in } \\
\text { this machine and sent through the various catheters shown into the } \\
\text { patient }\end{array}$ & 11 \\
\hline 1.7 & $\begin{array}{l}\text { Linear quadratic model representation of high and low LET radiation } \\
\text { causing cell death }\end{array}$ & 12 \\
\hline 2.1 & The electromagnetic spectrum & 16 \\
\hline 2.2 & $\begin{array}{l}\text { Probability of each of the three major photon interactions occurring for } \\
\text { various incident photon energies and target atomic numbers }\end{array}$ & 19 \\
\hline 2.3 & The three light charged particle interactions in matter & 20 \\
\hline 2.4 & Path shape for light and heavy charged particles traversing matter & 22 \\
\hline 2.5 & $\begin{array}{l}\text { Sample calculation of neutron RBE relative to x-rays using cell survival } \\
\text { curves. Adapted from Sorensen, B.S. }\end{array}$ & 25 \\
\hline 2.6 & $\begin{array}{l}\text { Low vs high LET radiation tracks. Low LET radiation, shown on the } \\
\text { track above, is less likely to cause irreparable DNA damage to the cell. }\end{array}$ & 27 \\
\hline 2.7 & $\begin{array}{l}\text { RBE shown as a function of increasing LET. The maximum RBE occurs } \\
\text { when LET is equal to } 100 \mathrm{keV} / \mu \mathrm{m}\end{array}$ & 28 \\
\hline 2.8 & $\begin{array}{l}\text { Plastic scintillator emitting its characteristic blue light (Eljen } \\
\text { Technologies, Sweetwater TX) }\end{array}$ & 33 \\
\hline 2.9 & $\begin{array}{l}\text { The W2 detector produced by the radiotherapy equipment company, } \\
\text { Exradin. }\end{array}$ & 33 \\
\hline 3.1 & $\begin{array}{l}\text { Schematic of radiation source incident upon a water phantom. Scoring } \\
\text { planes placed at two depths for each beam, electron energy spectra } \\
\text { were scored at all positions in the plane. }\end{array}$ & 41 \\
\hline 3.2 & $\begin{array}{l}\text { The } y \text { parameter as a function of } x \text {. This quantity represents the } \\
\text { probability of a lesion occurring given that two energy depositions occur }\end{array}$ & 41 \\
\hline
\end{tabular}




\begin{tabular}{|c|c|c|}
\hline & some distance $(\mathrm{x})$ apart for a given electron & \\
\hline 3.3 & $\begin{array}{l}\text { Summation of the energy spectra generated by the } 1 \times 1 \mathrm{~cm}^{2} \text { beam to } \\
\text { produce a composite } 10 \times 10 \mathrm{~cm}^{2} \text { field size }\end{array}$ & 44 \\
\hline 3.4 & $\begin{array}{l}\text { Variation in } \mathrm{RBE}_{\mathrm{M}} \text { at various positions surrounding a } 10 \times 10 \mathrm{~cm}^{2}(\mathrm{~A}) \text { and } \\
1 \times 1 \mathrm{~cm}^{2}(\mathrm{~B}) 6 \mathrm{MV} \text { beam. The } \overline{\alpha / \beta} \text { calculated for each voxel was } \\
\text { normalized to the central axis } \overline{\alpha / \beta} \text {. Variability curves are shown at } \\
\text { depths of } 1.5 \mathrm{~cm} \text { and } 5 \mathrm{~cm} \text {. The RBE } \mathrm{M} \text { profile in the in-plane and cross- } \\
\text { plane direction for } 10 \times 10 \mathrm{~cm}^{2} \text { (C). Comparison of RBE variation in the } \\
\text { composite } 10 \times 10 \mathrm{~cm}^{2} \text { field and the single } 10 \times 10 \mathrm{~cm}^{2} \text { field at a depth of } \\
1.5 \mathrm{~cm} \text { (D). }\end{array}$ & 45 \\
\hline 3.5 & $\begin{array}{l}\text { Normalized } \mathrm{RBE}_{\mathrm{M}} \text { values for the composite and single } 10 \times 10 \mathrm{~cm}^{2} \\
\text { plotted along with their corresponding electron fluence at various } \\
\text { positions at a depth of } 5 \mathrm{~cm} \text {. The dashed lines represent the normalized } \\
\text { electron fluence (all energies) present in both cases (blue: composite } \\
\text { field, black: single beam). The markers represent the normalized RBE } \\
\text { for both cases (blue: composite field, black: single beam). }\end{array}$ & 46 \\
\hline 3.6 & $\begin{array}{l}\text { Taking into account the findings of this work, the effective dose to the } \\
\text { organ at risk, particularly in cases where the organ at risk is in close } \\
\text { proximity to the tumour, may be larger than anticipated, and may } \\
\text { become significant especially if the OAR dose is pushed to near- } \\
\text { tolerance. In the schematic diagram above such a case is shown } \\
\text { although commonly a much greater number of beams are used. }\end{array}$ & 52 \\
\hline 4.1 & $\begin{array}{l}\text { Energy weighted photon spectra for }{ }^{125} \mathrm{I} \text { (blue; dotted), }{ }^{192} \mathrm{Ir} \text { (red; } \\
\text { dashed), and }{ }^{169} \mathrm{Yb} \text { (yellow; solid) from Taylor et al. The radioactive } \\
\text { source geometry surrounded by water was recreated in Geant } 4 \text {; the } \\
\text { data shown above was used as the photon emission spectra. }\end{array}$ & 63 \\
\hline 4.2 & $\begin{array}{l}\mathrm{RBE}_{M} \text { shown for }{ }^{125} \mathrm{I} \text { when }{ }^{192} \mathrm{Ir}(\text { at } x=0 \mathrm{~cm}) \text { was used as the reference } \\
\text { radiation. Error bars are representative of the standard error of the } \\
\text { mean } \mathrm{RBE}_{M} \text { calculated using multiple }(\mathrm{n}=5) \text { simulations with different } \\
\text { starting seed numbers. }\end{array}$ & 69 \\
\hline 4.3 & $\begin{array}{l}\mathrm{RBE}_{\mathrm{M}} \text { as a function of distance from the source }(x=0 \mathrm{~cm}) . \text { Data for } \\
\text { three sources are shown above, }{ }^{125} \mathrm{I}\left(\text { blue), }{ }^{192} \mathrm{Ir}(\mathrm{red}), \text { and }{ }^{169} \mathrm{Yb}\right. \\
\text { (yellow). For each source, the electron fluence present in the voxel at } \\
\text { position, } x, \text { was used as the test radiation, and the electron fluence at } \\
\text { the position } x=0 \mathrm{~cm} \text { (source) was used as the reference radiation. } \\
\text { Error bars are representative of the standard error of the mean } \mathrm{RBE}_{\mathrm{M}} \text { at } \\
\text { each position calculated using multiple }(\mathrm{n}=5) \text { simulations, each with } \\
\text { different initial seed numbers. }\end{array}$ & 70 \\
\hline 4.4 & $\begin{array}{l}\text { Photon and electron energy fluence spectra at the source position } \\
\text { (dotted line) and } 8 \mathrm{~cm} \text { (solid line) in water. The electron fluence spectra } \\
{ }^{192} \text { Ir and }{ }^{169} \mathrm{Yb} \text { are different when comparing the source position and the } \\
\text { periphery (resulting in the increased RBE } \mathrm{M} \text { ). Axes were kept consistent } \\
\text { in order to compare spectra between the three sources. }{ }^{125} \mathrm{I} \text { mean } \\
\text { electron energy for the source position was } 28.5 \mathrm{keV} \text {; at the periphery } \\
(8 \mathrm{~cm} \text { away) this increased to } 28.7 \mathrm{keV} \text {. }\end{array}$ & 71 \\
\hline
\end{tabular}




\begin{tabular}{|c|c|c|}
\hline 4.5 & $\begin{array}{l}\text { (A) The resulting }{ }^{192} \text { Ir HDR prostate treatment plan's electron fluence in } \\
\text { a slice bisecting the PTV. Bright spots indicate a high number of } \\
\text { electrons present (maximum of } 5 \times 10^{8} \text { ) while dark spots indicate few or } \\
\text { no electrons. (B) Single slice } \mathrm{RBE}_{M} \text { distribution calculated using the } \\
\text { electron fluence shown in Figure } 5 \mathrm{~A} \text {. RBE } \mathrm{RB}_{M} \text { values of } 1.00 \text { represent no } \\
\text { change relative to the source position; } \mathrm{RBE}_{\mathrm{M}} \text { of } 1.036 \text { represents a } \\
3.6 \% \text { increase. A projection of the } 2 \text {-dimsional } \mathrm{RBE}_{\mathrm{M}} \text { distribution is } \\
\text { shown in the } X-Y \text { plane. }\end{array}$ & 72 \\
\hline 4.6 & $\begin{array}{l}\text { Photon interaction cross sections in water obtained from the NIST } \\
\text { XCOM database. The average energy of the three radioisotopes } \\
\text { examined is highlighted. Pair production not shown due to zero } \\
\text { probability at the energy range shown. }\end{array}$ & 75 \\
\hline 5.1 & $\begin{array}{l}\text { (A) Specialized detector housing (shown in grey). This allowed for the } \\
\text { scintillator to be coupled to the flat-cleaved optical fiber for light } \\
\text { collection. The virtual water cap (shown left) allows for insertion into } \\
\text { clinically used virtual water slabs. The coin is shown for scale and has a } \\
\text { diameter of } 18.03 \mathrm{~mm} \text {. (B) Measurement set-up in the Orthovoltage x- } \\
\text { ray unit; the scintillator detector was placed at a depth of } 1.5 \mathrm{~cm} \text { in } \\
\text { water. }\end{array}$ & 86 \\
\hline 5.2 & $\begin{array}{l}\text { Measured scintillator response. The light signal as read by PMT } \\
\text { (charge, nC) normalized to absorbed dose to water at point of } \\
\text { measurement (at } 1.5 \mathrm{~cm} \text { depth for each respective beam). Response is } \\
\text { shown as a function of mean collisional stopping power determined } \\
\text { using the NIST ESTAR database. The subplot is showing an enlarged } \\
\text { representation of the response to low stopping power (electron beam) } \\
\text { radiation at the point where the impact of doping becomes insignificant. } \\
\text { The mean collisional stopping power shown on the x-axis was obtained } \\
\text { as explained in Section 5.3.3. }\end{array}$ & 93 \\
\hline 5.3 & $\begin{array}{l}\text { Optimal kB values that result in agreement between Monte Carlo } \\
\text { scintillation and measurement results. The scintillator light yield }\left(\mathrm{L}_{0}\right) \\
\text { used in the simulation is also shown in this figure (secondary } \mathrm{y} \text {-axis). } \mathrm{kB} \\
\text { values are presented using units of } \mathrm{mm} / \mathrm{MeV} \text {, while light yield is shown } \\
\text { in photon/MeV; both sets of data are shown as a function of the } \\
\text { effective atomic number of each scintillator. The 'kB Curve Fit' values } \\
\text { are also shown as a function of effective } \mathrm{Z} \text { of the scintillator material; } \\
\text { curve fitting of the data was done using MATLAB. The corresponding } \\
\text { lead doping percentages are shown below the } \mathrm{x} \text {-axis. }\end{array}$ & 96 \\
\hline 5.4 & $\begin{array}{l}\text { Measured response of the differently lead-doped scintillators to mean } \\
\text { energy of electrons in the scintillator volume. For each scintillator, its } \\
\text { response at a given energy is normalized to its response to the } 15 \mathrm{MeV} \\
\text { electron beam (average electron energy at } 1.5 \mathrm{~cm} \text { was approximately } 8 \\
\mathrm{MeV} \text { ). Measured results are shown as solid lines, while simulated } \\
\text { results use dotted lines. All measured data shown was obtained using }\end{array}$ & 97 \\
\hline
\end{tabular}




\begin{tabular}{|c|c|c|}
\hline & $\begin{array}{l}\text { the scintillators obtained from Eljen Tech., although two different } \\
\text { manufacturers were tested to ensure reproducibility (Section 5.3.2). }\end{array}$ & \\
\hline 6.1 & $\begin{array}{l}\text { (A) Scintillator measurement setup - for each measurement, } \\
\text { the scintillators were housed in a detector covering made of polyethyl } \\
\text { ether ketone (PEEK) to achieve water equivalence. This unit (shown in } \\
\text { beige) is then housed in a virtual water cap (shown in brown, far left) } \\
\text { which inserts into the clinical QA virtual water slabs. Canadian dime } \\
\text { shown for scale. (B) CAD Design for the detector head shown in the } \\
\text { assembled configuration. The optical fiber (shown in blue) rests against } \\
\text { the scintillator face; the outer virtual water surface of detector has a } \\
\text { length of length is } 6 \mathrm{~cm} \text {, and a diameter of } 2.2 \mathrm{~cm} \text {. }\end{array}$ & 115 \\
\hline 6.2 & $\begin{array}{l}\text { (A) Scintillator measurements under } 4 \text { different orthovoltage beams. } \\
\text { Measurements were conducted using } 100,180,250 \text {, and } 300 \mathrm{kVp} \text { x-ray } \\
\text { beams using a } 30 \mathrm{~cm} \text { source to surface distance open cone with a } 10 \\
\mathrm{~cm} \text { diameter. (B) Scintillator measurements using the HDR }{ }^{192} \mathrm{Ir} \text { source. } \\
\text { The detector was placed at a depth of } 1.5 \mathrm{~cm} \text { from the surface of the } \\
\text { virtual water slab, and the HDR source was placed at the surface. }\end{array}$ & 116 \\
\hline 6.3 & $\begin{array}{l}\text { Integrated Birks' Law shown for increasing LET in water. Collisional } \\
\text { stopping power values were obtained using the NIST ESTAR database, } \\
\text { and the appropriate scintillator dependent parameters were chosen to } \\
\text { calculate the predicted light output, dL/dx. }\end{array}$ & 121 \\
\hline 6.4 & $\begin{array}{l}\text { Total photon attenuation cross sections for each of the four scintillators } \\
\text { used obtained from the NIST XCOM Database }\end{array}$ & 122 \\
\hline 6.5 & $\begin{array}{l}\text { Electron LET spectra for each beam at } 1.5 \mathrm{~cm} \text { depth generated using } \\
\text { Monte Carlo GEANT4. }\end{array}$ & 122 \\
\hline 6.6 & $\begin{array}{l}\text { Electron LET spectra determined using the method described } \\
\text { (diagonal) compared to the Monte Carlo predicted spectra (solid) shown } \\
\text { for the } 5 \text { x-ray beams examined. The error bars shown with the } \\
\text { 'method' data correspond to the total uncertainty calculated using the } \\
\text { uncertainty analysis shown in Table } 6.4 \text {. The error bars shown for the } \\
\text { Monte Carlo data correspond to the absorbed dose uncertainty after } \\
\text { changing the starting simulation seed multiple times }(n=5) \text {. }\end{array}$ & 123 \\
\hline
\end{tabular}




\section{Introduction}

\subsection{Cancer}

Cancer is one of the most notorious and prevalent illnesses in the modern healthcare landscape. This illness can be defined as the uncontrolled growth and division of cells, eventually amassing into a volume referred to as a tumour. Tumours can be benign, where they remain in one location and cancer does not spread into surrounding tissues and organs - or they can be malignant. Once it has become malignant, cancer becomes very difficult to treat because the cells which are growing uncontrollably have spread to different regions of the body through the blood and lymphatic systems. ${ }^{1}$

In properly functioning cells, the genetic instructions which predetermine their function and biological destiny (DNA) are replicated, and then a process called mitosis occurs. During mitosis, the cell's DNA and functional components, such as various organelles, are divided into two, separate, identical daughter cells. This allows the body to continuously and quickly replace dying cells. Unfortunately, in cancerous cells, the genetic code becomes damaged or mutated. These disastrous mutations prevent the cell from differentiating into what it should have become. Also, instead of quickly dying 
due to being dysfunctional, the mutations cause the cell to grow and divide rapidly, consuming important resources from other cells. ${ }^{2}$

According to the Canadian Cancer Society, cancer is responsible for $30 \%$ of all adult deaths and is the leading cause of death in Canada. In 2017, there were an estimated 206,200 new cases among Canadians along with 80,800 deaths. Of these cases, the most prevalent were prostate ( $21 \%$ of men), lung ( $14 \%$ of all new cases), breast (25\% of women), and colorectal ( $13 \%$ of all new cases) cancers. These grim statistics provide a glimpse into the severity and prevalence of this disease. The Canadian Cancer Society estimates that $60 \%$ of Canadians are expected to survive for at least 5 years post diagnosis, however this value is highly dependent upon the type of cancer. For example, the 5 year survival rate for prostate cancer is $95 \%$ while only $17 \%$ for lung cancer. ${ }^{3}$

There are three main avenues of cancer treatment: surgery, chemotherapy, and radiation therapy. Once referred to the cancer center by the patient's family physician, a team of physicians which include medical, radiation, and surgical oncologists determine the best course of treatment in each individual case. There are several other less prevalent treatment methodologies which include hormonal therapy, immunotherapy, thermal therapy, and stem cell transplantation. Often, the patient's treatment plan will consist of a combination of the treatment methods listed above.

\subsection{Radiation Therapy}


Radiation therapy, also called radiotherapy, is used in approximately $50 \%$ of cancer cases in the United States. ${ }^{4}$ This highly technical treatment option directs high energy radiation towards the tumour in order to cause cell death. The main method by which radiation causes cells to die is by causing damage to DNA such that the cell is not able to properly replicate; this is called mitotic cell death. ${ }^{2}$ Despite the fact that cells possess complex and effective repair mechanisms, by delivering high amounts of radiation, cell death is inevitable. It is important to note that radiation inherently cannot discriminate between healthy and cancerous tissue. Thus, the greatest challenge in this field is directing radiation towards the tumour and away from healthy tissue. The latter is of utmost importance because increased radiation exposure to healthy tissues can actually cause the development of cancer. Radiation therapy can be divided into two main types: external beam and internal beam. External beam radiotherapy involves an external beam source producing radiation directed towards the patient. In the external beam case, radiation must pass through any tissue that exists between the surface of the patient and the location of the tumour, resulting in the exposure of healthy tissues. Internal radiotherapy is more specifically referred to as brachytherapy, 'brachy' meaning 'short distance' in Latin. In this type of radiotherapy, a radioactive source is surgically implanted to be inside or adjacent to the tumour volume. This results in minimal radiation exposure to healthy tissues, but carries with it the complications and risks associated with surgery.

\section{External beam radiotherapy: Photon and electron beams}


The most common type of radiotherapy is the use of high energy photons produced by a linear accelerator or by a radioisotope. Historically, this type of radiotherapy was referred to as 'teletherapy'. In order to explain the use of photons in radiotherapy, the particle-like behavior in the photon's wave-particle dual nature must be considered. From this perspective, photons are mass-less particles which indirectly ionize tissue. The 'indirect' classifier is used to convey the fact that the photon eventually sets a charged particle in motion (mechanisms of this phenomena are discussed in Section 2.1), which then causes damage via ionization to occur. ${ }^{5}$ Directly ionizing radiation, which includes particles like electrons and protons, does not require a charged particle intermediate. In cancer radiotherapy, high energy photons are typically used. These photons are typically above $1 \mathrm{MeV}$ in energy and whether the radiation source is a linear accelerator or a radioisotope, complex equipment is required. At this point, it is important to note that photons produced by electronic interactions are referred to as x-rays, while photons produced via nuclear interactions are called gamma rays. ${ }^{6}$

Linear accelerators used in radiotherapy produce photons ranging in energy from 4 to $21 \mathrm{MeV}$ (Figure 1.1). In this machine, electrons are first produced in a vacuum by the process of thermionic emission. These electrons are then accelerated to high energies using a strong electric field and waveguide. Once they reach the desired energy, these electrons are directed towards a high atomic number target in which a radiative collision occurs. By this interaction, a high energy photon, called a Bremsstrahlung $\mathrm{x}$-ray, is produced and directed toward the patient. This photon 
undergoes several levels of collimation to ensure a high level of conformity to the patient's tumour.

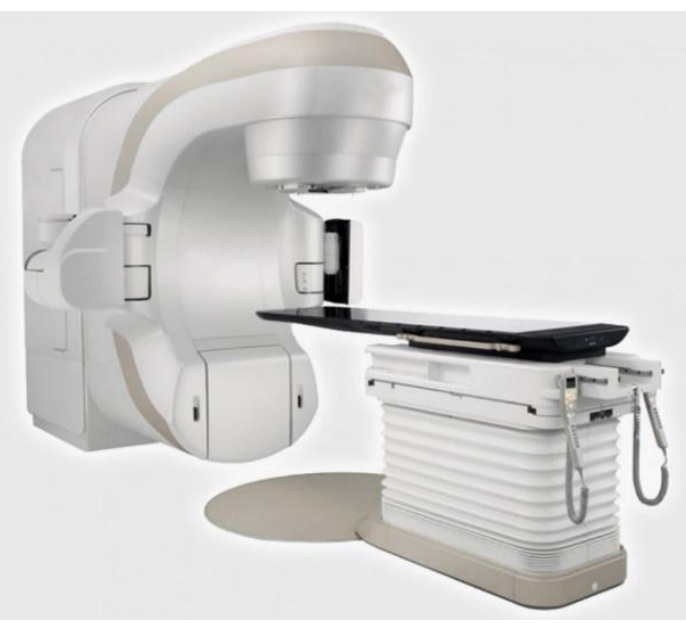

Figure 1.1: Typical photo of a linear accelerator used to deliver high energy photon radiotherapy to cancer patients. ${ }^{7}$

Recently, there have been several new developments in linear accelerator-based photon therapy. The most prevalent example of photon therapy advancement is the development of intensity modulated radiation therapy, or IMRT. In this technique, radiation is delivered from many different angles causing the healthy tissue irradiation from each angle to be minimal, but the accumulated dose at the focal point (the tumour) to be significant. At each beam delivery angle, there are small collimators called 'multileaf collimators' or MLCs which enter into the path of photons and cause the beam to be shaped according to the tumour.

Another recent development is the use of magnetic resonance imaging during radiation therapy. Currently, patients are imaged using CT at the beginning of their treatments and the radiation treatment plan is developed based on that. Organ movement during therapy, or patient anatomical changes due to weight loss, bowel 
movements, and gas are not accounted for. The goal of image guidance is to track all variations in patient anatomy and movement during their treatment. The increased confidence about anatomical positioning allows for increased amounts of radiation to be delivered. The machines which deliver this MRI-guided radiation therapy are highly specialized (Figure 1.2) because the presence of a strong magnetic field has a significant impact on the behavior of electrons in the patient which must be accounted for.

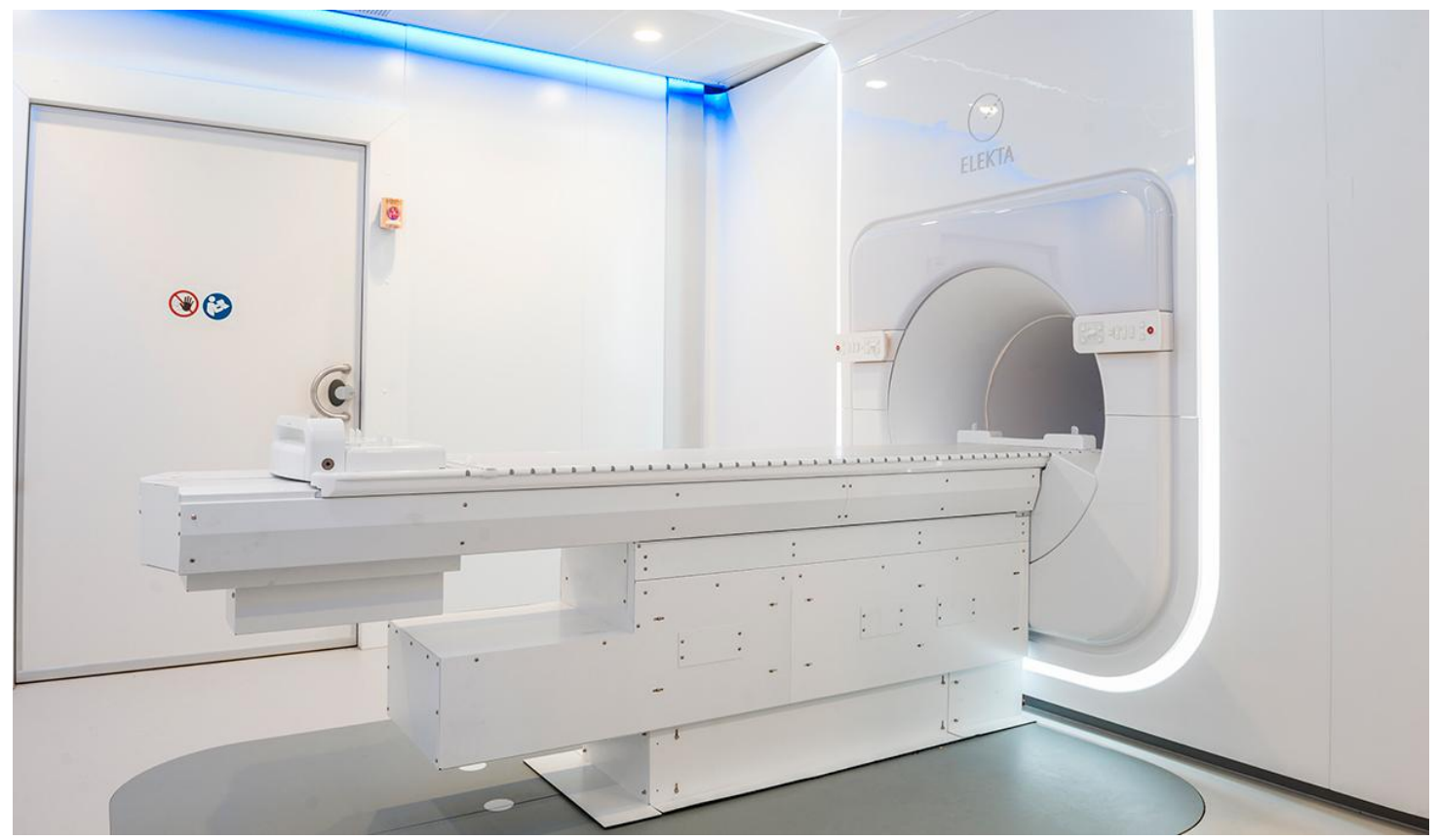

Figure 1.2: The MR-linac produced by Elekta, housed at Sunnybrook Health Sciences Centre. $^{8}$

The unstable isotopes used as a radiation source in some radiotherapy treatments occur naturally and can also be produced artificially. Briefly, radioisotopes exist when an atom contains an excess number of neutrons, making it unstable. In several radioisotopes, excess energy is emitted in the form of a high energy photon; 
these photons are referred to as gamma rays. There have historically been several different radioisotopes used in external beam therapy; these include: cobalt-60, radium226, and cesium-137. Due to its higher energy and long half-life (5.27 years), cobalt-60 has been most commonly used.

The cobalt-60 radioisotope emits several different gamma (photon) and beta (electron) rays during its decay (Figure 1.3). This radioisotope is produced through the irradiation of stable cobalt-59 in a nuclear reactor. The decay process results in the emission of two photons of 1.17 and $1.33 \mathrm{MeV}$ in energy, per decay. Radioisotopebased external beam radiotherapy has mostly been replaced by linear accelerators for several reasons. Firstly, a radioactive source such as cobalt-60 is constantly emitting radiation. This means that the source housing and entire treatment machine must be designed keeping in mind that even when patients are not being treated, radiation is being emitted. Secondly, the potential use of radioisotopes in the production of 'dirty bombs' for large scale attacks is a growing fear. ${ }^{9}$ By using radioisotopes such as cobalt60 , several additional security layers must be added to the hospital's infrastructure to remain in compliance with regulatory commissions. ${ }^{10}$

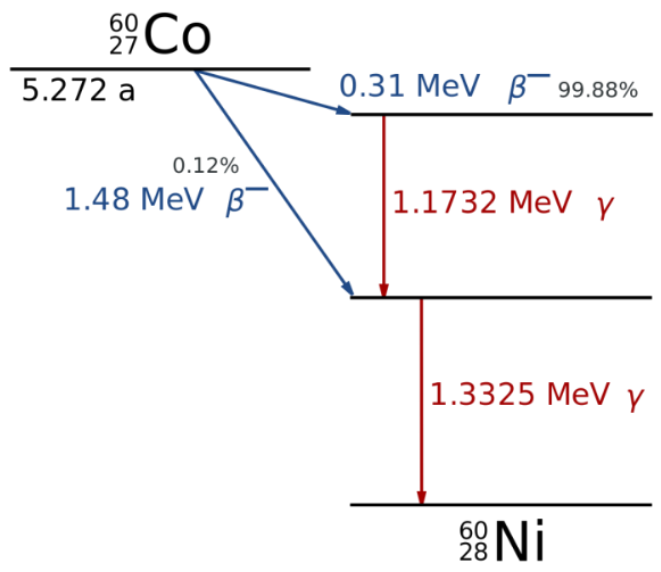


Figure 1.3: Decay scheme of cobalt-60 [WikiCommons Image]

High energy photons produced by radioisotopes such as cobalt-60 are still used in several specialized machines. For example, the GammaKnife (Figure 1.4) produced by Elekta ${ }^{\mathrm{TM}}$ is a specialized unit developed for treating brain cancers. This machine houses 192 high activity cobalt-60 sources allowing for the delivery of high doses to the tumour while minimizing radiation delivered to healthy areas of the brain. ${ }^{11}$

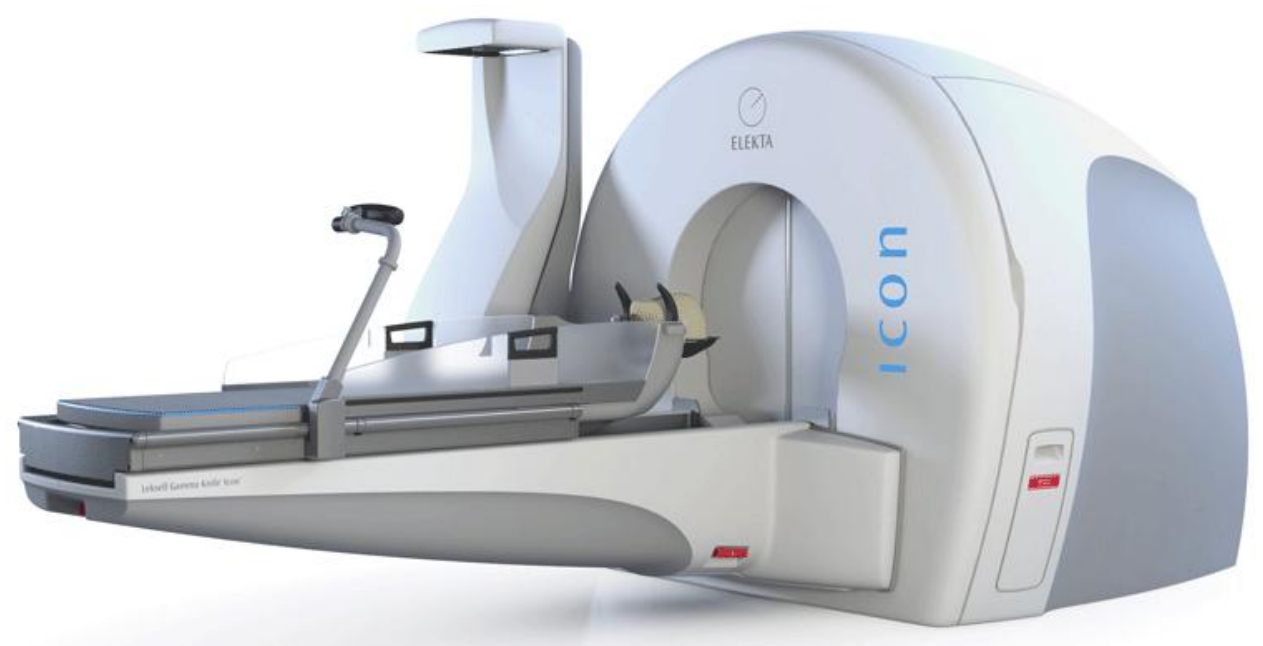

Figure 1.4: Leksell GammaKnife produced by Elekta. ${ }^{12}$

Linear accelerators can also be used to produce high energy electron beams. The electron that is accelerated by the waveguide can be directed towards the patient without the use of a high-Z target. Electron beam therapy is not commonly used since electrons will damage all tissue between the surface of the patient and the tumour. Thus, it is mostly used for skin and superficial cancer treatments. 


\section{External beam radiotherapy: Particle beams}

Radiation therapy can also be delivered using heavy, charged particles such as protons and carbon-ions. This allows for sharper radiation dose distributions around the tumour. When a proton enters tissue, it begins dissipating its energy right away. At the end of its track, the proton will deposit most of its energy in a location called the Bragg Peak (Figure 1.5). After this region, no further energy is deposited in tissue and the proton has stopped. Photon therapy exhibits an exit dose due to the stochastic nature of photon interactions - this means that healthy, non-cancerous tissue behind the tumour is also receiving radiation. In Figure 1.5, the relative depth dose distribution is shown. As radiation travels through tissue, the energy it dissipates is absorbed. By normalizing the energy absorbed to the mass of that tissue, the absorbed dose is obtained. The relative dose distribution shown in Figure 1.5 is obtained by normalizing the dose at all positions to the maximum dose. The harmful effects of particle therapy include possibly inducing cancer and deterministic effects such as skin burns.

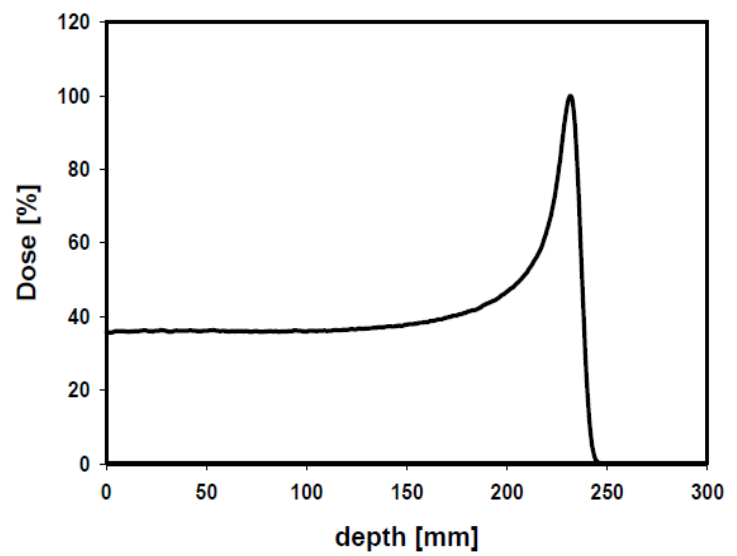


Figure 1.5: Depth vs dose distribution for a proton travelling through water. The proton deposits the largest amount of energy at the end of its track (Bragg Peak). ${ }^{13}$

\section{External beam radiotherapy: Orthovoltage}

Superficial skin treatments can be done using an orthovoltage unit. This machine emits x-rays ranging from 100 to $300 \mathrm{kVp}$. X-rays are produced using an $\mathrm{x}$-ray tube operating at high voltages (up to $300 \mathrm{~V}$ ). Electrons are accelerated through a strong electric field and their eventual collision with the Tungsten target results in the production of $\mathrm{x}$-rays. ${ }^{14}$ These $x$-rays are then filtered using various compositions of high atomic number materials such as lead and tungsten in order to achieve the desired beam energy. Given the lack of popularity and low energies used, most hospitals do not use software for orthovoltage treatment planning. ${ }^{15}$

\section{Internal beam radiotherapy: Brachytherapy}

Brachytherapy is the surgical implantation of radionuclides adjacent to or inside the cancerous regions. This technique has achieved an excellent dose distribution around the tumour, however, there are several challenges to consider in brachytherapy. These include the risks when dealing with surgery, radionuclides, and radionuclide positioning. The reason why excellent dose distributions are achieved is that typically low energy sources are used, preventing radiation from travelling unnecessarily far. Brachytherapy can be divided into two main types: high dose rate (HDR, > 12 Gy per hour) and low dose rate (LDR, 0.4 to 2 Gy per hour)..$^{5}$ In high dose rate brachytherapy, 
a high activity source is used to irradiate the tumour. This procedure is done by inserting catheters inside the patient's tumour and connecting an after-loader machine to these catheters (Figure 1.7). Using a patient-specific treatment plan, the high activity source travels out of the after-loader, through the catheters, and into the patient. The treatment is modulated by adjusting the radionuclide's dwell time as a function of position in the patient.

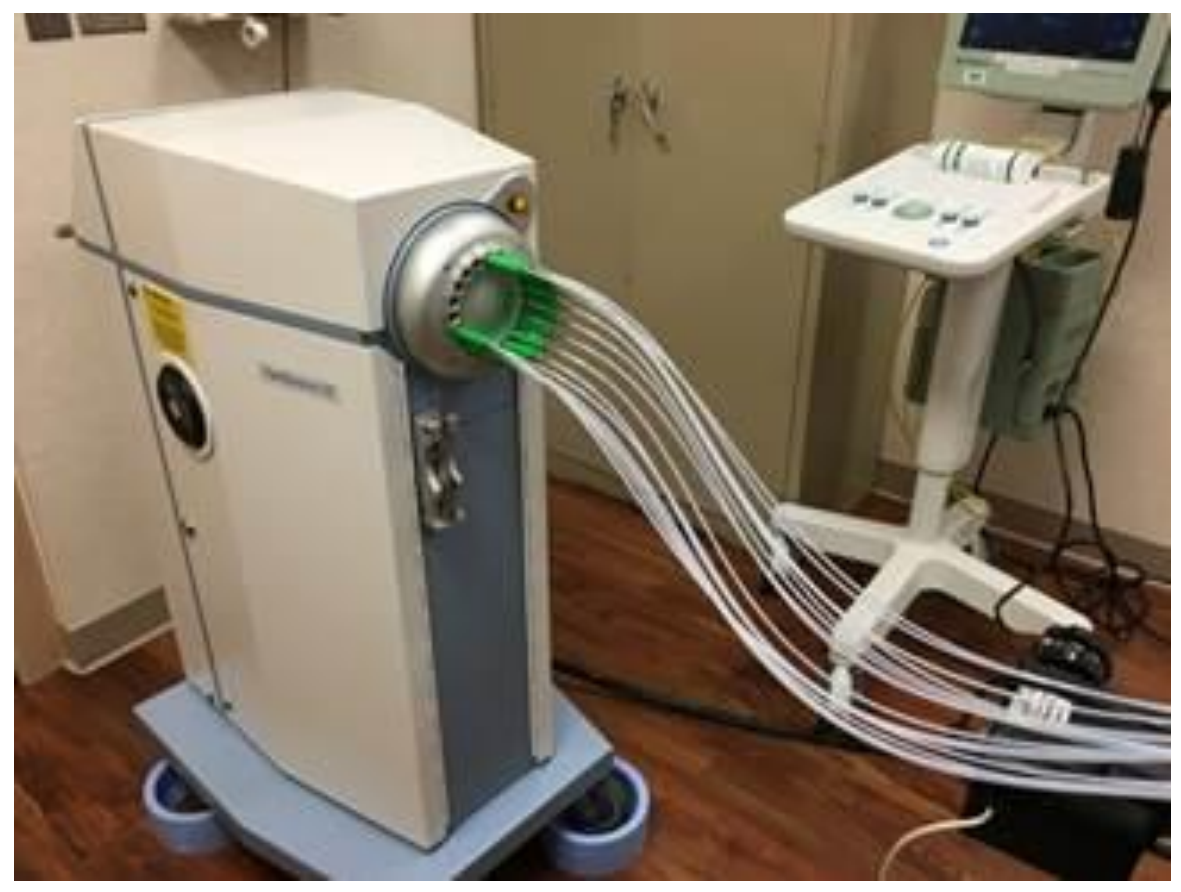

Figure 1.6: HDR brachytherapy after-loader. The high activity source is housed in this machine and sent through the various catheters shown in the figure into the patient. ${ }^{16}$

\subsection{Rationale and Significance}

In the radiation therapy modalities described above, the amount of radiation to be delivered is determined using a quantity called absorbed dose. As briefly discussed 
above, absorbed dose is the amount of energy absorbed in tissue per unit mass. Radiation therapy treatment planning is based on the assumption that absorbed dose and biological damage correlate; increased dose should result in increased cell death. There are several tools which predict cell survival as a function of absorbed dose.

The most commonly used tool for predicting cell survival is the linear-quadratic model (Figure 1.8). In this model, the surviving fraction of cells is described as a function of absorbed dose. The model also distinguishes between high and low linear energy transfer radiation. The linear energy transfer, or LET, is the amount of energy absorbed in tissue per unit length along a particle's path. Low energy electrons and protons fall into the medium LET category, while low LET radiation includes high energy photons and electrons.

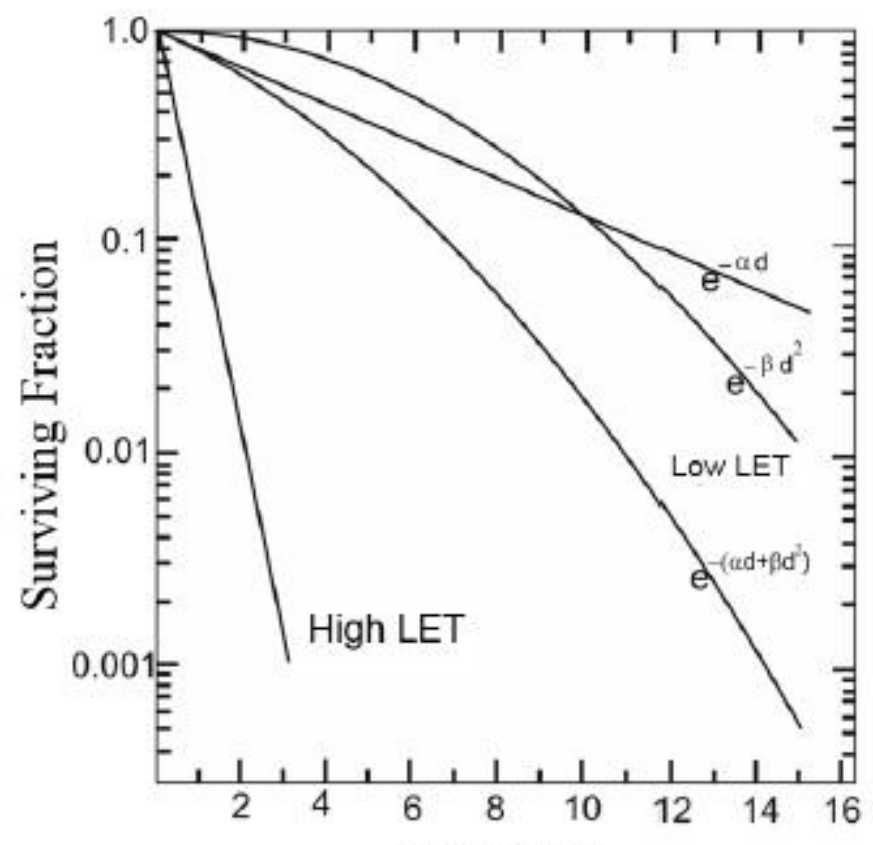

Dose (Gy)

Figure 1.7: Linear quadratic model representation of high and low LET radiation causing cell death. ${ }^{17}$ 
Currently, LET is not accounted for during photon and electron radiation therapy treatment planning. Essentially, during radiation therapy it is assumed that only high energy photons and electrons will be interacting with the patient, and the low energy, high LET component is negligible. With the advent of new technologies in radiation therapy, such as IMRT, the highly damaging component may be causing more damage

than previously thought. ${ }^{18-22}$ For particle therapies such as protons, a generic weighting factor of 1.1 is used. This means that in patients, it is assumed that protons are $10 \%$ more damaging than photons emitted by cobalt-60. Yet, several studies have shown that proton damage enhancement can vary depending on the position relative to the Bragg peak. ${ }^{23-25}$.

\subsection{Specific Aims}

In order for radiation therapy to utilize biological effectiveness-based treatment planning, clinically suitable 'radiation quality' detectors are required. Currently, no such detectors are available. The focus of this work was to first demonstrate the need for these detectors in external beam photon radiotherapy and brachytherapy, and then to develop a 'radiation quality meter' using differently doped plastic scintillators. By having uniquely energy dependent plastic scintillators, the electron LET spectrum can be resolved. In this work, 'radiation quality' is defined as a generic term for information regarding the LET or the relative biological effectiveness (RBE) of a radiation treatment beam. 


\section{Biological effect variation in radiation therapy}

Monte Carlo simulation and advanced microdosimetric techniques were used to evaluate how relative biological effectiveness changes in external beam photon radiotherapy and brachytherapy.

\section{Development of a 'radiation quality meter'}

Doped plastic scintillators were characterized in several low and medium LET radiation beams. These plastic scintillators had not been previously characterized or used in any radiotherapy settings. Once characterized, a detection method and signal processing algorithm were developed to resolve the electron energy spectrum of a radiation beam from the scintillator signal.

\section{Radiation quality measurement}

Using the radiation quality meter, the 'radiation quality' was measured in several medium and low LET radiation beams. These include several orthovoltage $\mathrm{x}$-ray beams, high energy photon and electron beams, and an iridium-192 HDR brachytherapy source.

\subsection{Thesis Organization}

In Chapter 2, the physics behind fundamental radiation interactions of interest, Monte Carlo Simulation technique, as well as plastic scintillation will be discussed. Chapter 3 examines the change in maximum RBE in open and composite fields under a $6 \mathrm{MV}$ linear accelerator photon beam. Chapter 4 examines maximum RBE variation in 
three different radionuclides (iridium-192, ybettrium-169, and iodine-125) and the impact of this variation on an HDR prostate treatment plan. Chapter 5 describes the characterization of differently lead doped plastic scintillators in medium and low LET beams, and presents a new equation describing doped plastic scintillator response. Chapter 6 demonstrates the measurement of the electron LET spectra in various medium and low LET radiation beams. Chapter 7 summarizes our work in the development and use of this novel radiation quality meter. 


\section{Radiation Dosimetry}

The phenomenon of ionizing radiation is utilized in several different disciplines including radiotherapy, atomic energy, military applications, and space travel. ${ }^{26-28}$ Due to its widespread use and potential harmful effects, characterizing the amount of radiation is of utmost importance. The most commonly used quantity in order to achieve this is called absorbed dose, formally defined as the amount of radiation energy absorbed per unit mass in a medium. In order to further understand this, the passage of ionizing radiation through matter must be described in depth.

The 'ionizing' classifier when referring to radiation is simply used to distinguish between particles that are travelling fast enough to remove an orbital electron from a target atom and particles that are not. ${ }^{2}$ Henceforth, the term 'radiation' will refer specifically to ionizing radiation since there was not a non-ionizing component to this research project.

There are two different types of radiation which have the capacity to ionize matter: electromagnetic radiation and particulate radiation. Ultimately, energy deposition in matter occurs through the interactions of charged particles. Thus, when considering 
electromagnetic radiation, the energy transferred to charged particles is of utmost importance.

\subsection{Electromagnetic Radiation}

Electromagnetic radiation exists in a spectrum (Figure 2.1); wavelengths shorter than $10^{-8} \mathrm{~m}$ or greater than $124 \mathrm{eV}$ in energy are typically in the ionizing range. ${ }^{2}$ Photons passing through matter can either disappear completely and impart their energy to light charged particles or scatter off with a lower energy and new trajectory. These photons, frequently referred to as x-rays and gamma rays, deposit their energy via three main interactions: 1) the Compton effect, 2) the photoelectric effect, and 3) pair production. Other possible interactions such as Thomson and Rayleigh scattering, triplet production, and photonuclear reactions rarely occur at the energies we are considering and when they do, there is no deposition of energy into the medium from the incoming particle. $^{5}$

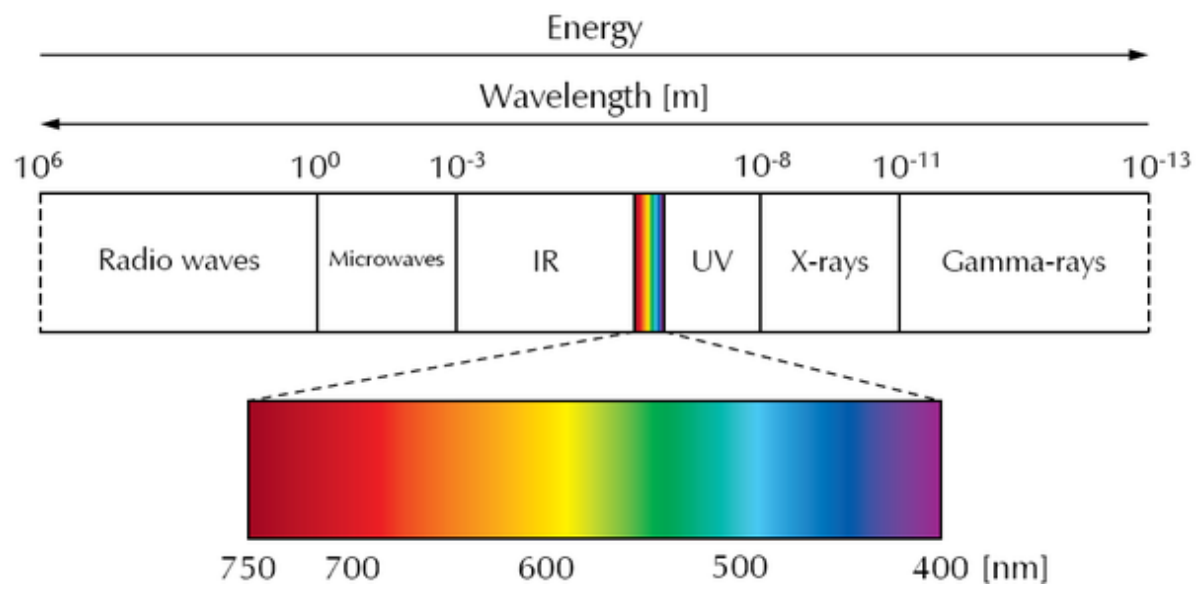

Figure 2.1: The electromagnetic spectrum. ${ }^{2}$ 
The first of the three listed photon interactions, the Compton effect, occurs when an incoming photon collides with a loosely bound electron, causing both particles to scatter post-collision in new trajectories. Thus, this interaction is also known as 'incoherent scattering'. For the scope of this thesis, it is important to know that the Compton effect is the dominant interaction in the therapeutic energy range.

The second important photon interaction is the photoelectric effect. This occurs when a photon with enough energy to ionize an atom interacts with a tightly bound electron. Here an incident photon collides with a tightly bound orbital electron causing it to be ejected out of the atom's electron cloud, leaving a hole behind. Conservation of energy dictates that in order to reach stability, an electron from a higher energy shell transitions down to fill the hole; this energy transition results in the emission of an x-ray. The x-ray emitted as a result of the electron's energy transition is called a characteristic $x$-ray. This name derives from the fact that the electron shell energies are unique to each element; each x-ray emitted as a result of this phenomena is characteristic in energy to its atom of origin. The likelihood of this interaction occurring as well as the energy of the photoelectron and characteristic x-ray emitted is highly dependent upon the shell in which the target electron resides, atomic number, and incoming photon energy. Characteristic $x$-rays refer to photons emitted by an atom as a result of electron transitions with its orbitals; since shell and subshell configurations are characteristic to each atom, the energy (in the form of an x-ray) released due to transitions is also characteristic to its atom of origin.

When considering the photoelectric effect, there are two different ways energy is transferred to charged particles: photoelectron emission and Auger electrons. The term 
'photoelectron' refers to a tightly bound electron which is liberated from the target atom by an incoming photon. The second term, Auger electrons, is used to describe when the exiting characteristic $x$-rays interact with a second orbital electron. It is possible that multiple subsequent Auger electrons are produced, this is referred to as the Auger cascade. It is important to note that the photoelectric effect is the dominant interaction in high-Z targets such as bone and at low energies $(<0.5 \mathrm{MeV})$.

The third and final noteworthy photon interaction in matter is pair production. This occurs when the incoming photon's energy is greater than the rest energies of the electron and positron (1.02 MeV). An incident photon becomes completely absorbed and energy is released in the form of electrons and positrons. The incoming high energy photon interacts with the field of the nucleus resulting in the production and release of an electron-positron pair. Another similar interaction is called electronic pair production, also known as triplet production, and it occurs when the incident photon has a higher energy and interacts with the electron field.

In summary, the Compton effect, photoelectric effect, and pair production can occur when an incident photon interacts with a target atom. Each of these interactions has an associated probability (cross-section) which is summarized in Figure 2.2. This figure demonstrates that at lower energies and high $Z$, the photoelectric effect dominates. As an example, this is the reason behind why the photoelectric effect is the dominant interaction in bones at $\mathrm{x}$-ray imaging energies. In the therapeutic photon energy range ( 1 to $6 \mathrm{MeV}$ ), the dominant interaction is the Compton effect. Pair production occurs at higher energies and dominates the high $\mathrm{Z}$ region. 


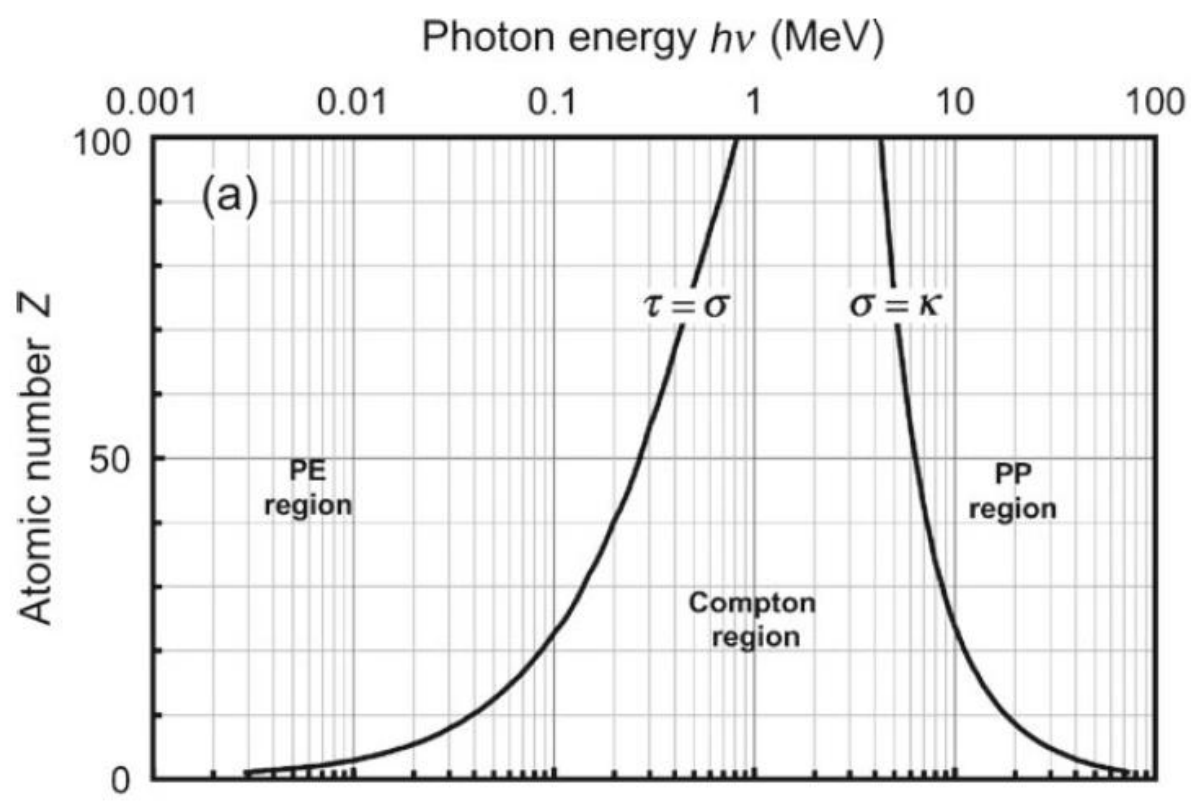

Figure 2.2: Probability of each of the three major photon interactions occurring for various incident photon energies and target atomic numbers. ${ }^{5}$

It is also important to distinguish between $\mathrm{x}$-rays and gamma rays. ${ }^{2,5}$ These two terms are used synonymously in popular culture, however, the difference between them is that x-rays are produced via electronic interactions and transitions, whereas gamma rays are produced solely as a result of changes in the nucleus. Thus, the photons used in imaging and linear accelerator-based radiotherapy are x-rays, and the photons emitted by cobalt-60 or caesium-137 are called gamma rays.

\subsection{Charged Particle Interactions}

As previously stated, the eventual damage caused by ionizing radiation to tissue is ultimately done by charged particles. When photons are the incident radiation, the light charged particles (electrons and positrons) freed as a result of the interactions 
discussed above go on to cause radiation damage to tissue. Often, the incident radiation itself is an electron, or in some specialized situations, a heavier particle such as a proton, alpha particle, or a carbon ion.

For light charged particles, there are three main types of collisions that may occur: hard collisions, soft collisions, and radiative collisions. ${ }^{5}$ Hard collision is when the incident electron and an orbital electron undergo a coulombic force interaction at a close distance. Soft collisions are similar to hard collisions; however, the interaction distance is much longer and therefore less energy is imparted onto the target atom's electron. The third type, radiative collisions, occurs when the incident electron accelerates due to coulombic interactions with the nucleus. When this acceleration occurs, a high energy photon, commonly referred to as a Bremsstrahlung photon, is released. These three interactions are summarized in Figure 2.3.

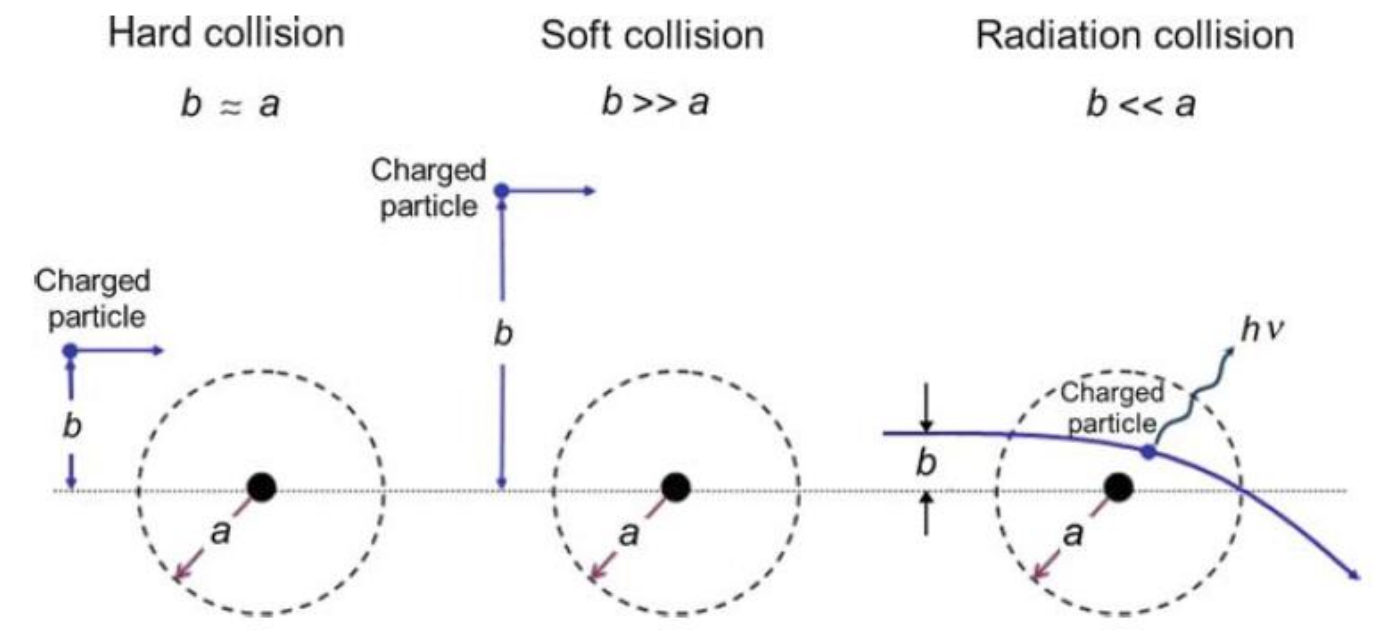

Figure 2.3: The three light charged particle interactions in matter. ${ }^{5}$

When hard collisions occur, the electron previously bound to the target atom leaves the region as a delta ray, which is an electron energetic enough to leave the 
local volume. Despite occurring rarely, hard collisions result in a very large amount of energy transfer from the incident electron. Soft collisions are distant coulombic interactions between the incident charged particle and an orbital electron. These are much more likely to occur relative to hard collisions and only a small amount of energy is transferred to the electron each time. An electron travelling will lose approximately $50 \%$ of its energy through soft collisions, and $50 \%$ through hard collisions.

\subsection{Stopping Power and Linear Energy Transfer}

In order to quantify how much energy is lost by a charged particle as it traverses through matter, the concept of stopping power is used. As discussed previously, whenever an electron passes through matter, it may interact via Coulombic forces with orbital electrons (soft and hard collisions) as well as the nucleus (radiative, or Bremsstrahlung interaction). The stopping power is defined as the amount of energy

dissipated by the particle per unit distance along the particle's path length. The density normalized stopping power is typically reported in the literature and is referred to as the

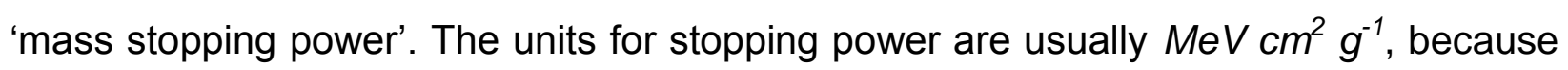
the energy loss per unit distance ( $\mathrm{MeV}$ per $\mathrm{cm}$ ) is normalized to the density $\left(\mathrm{g}_{\text {per }} \mathrm{cm}^{3}\right.$ ) of the target material. ${ }^{5}$

Stopping power (mathematically represented by $S$ ) can be further divided into charged particle interaction types (Equation 2.8). The collisional stopping power refers to the sum of energy losses as a result of hard and soft collisions per length along the particle's path. That is, energy lost by the particle due to collisions only will be represented through the collisional stopping power. The radiative stopping power 
represents the amount of energy lost by the initial charged particle due to Bremsstrahlung interactions and in-flight annihilation. For heavier charged particles, the radiative stopping power is zero because the Coulombic interaction with the nucleus is not strong enough to cause significant deviation or deceleration in the heavy charged particle's path. It is also worthwhile to note that heavy particles do not experience significant angular deflections during collisional interactions. This is in stark contrast to lighter charged particles and can be seen in Figure 2.4 below. Thus, the stopping power of charged particles in a medium is given by

$$
S_{\text {tot }}=S_{\text {col }}+S_{\text {rad }}=S_{\text {col }}^{\text {soft }}+S_{\text {col }}^{\text {hard }}+S_{\text {rad }}
$$

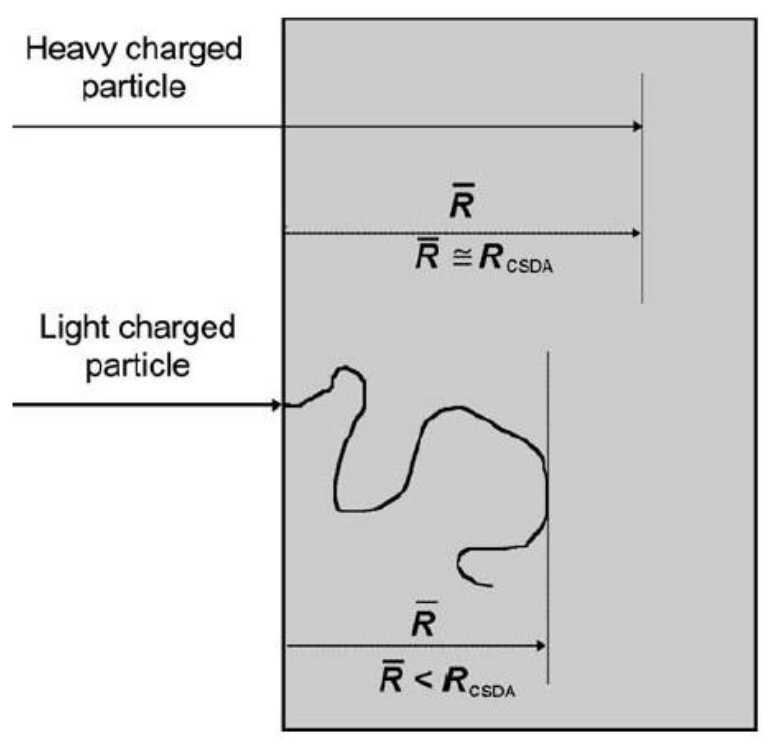

Figure 2.4: Path shape for light and heavy charged particles traversing matter. ${ }^{5}$

The Linear Energy Transfer, or LET, is another commonly used quantity in radiation dosimetry. ${ }^{29}$ Where stopping power is the energy 'lost' by the particle per unit 
length along the particle's path, the LET quantifies the energy 'absorbed' by the medium. Thus, the linear energy transfer is equivalent to the collisional stopping power but from the perspective of the medium rather than the particle. Hard collisions will often cause the target electron to be ejected from the local volume in the form of a delta ray. When defining LET, these non-locally absorbed electrons must be accounted for, thus delta thresholds are used. For example, an LET value reported with a threshold of 100 $\mathrm{eV}\left(L E T_{\Delta=100 \mathrm{eV}}\right)$ will be the energy absorbed locally from all electrons with energy less than $100 \mathrm{eV}$ - those with greater energies are considered to deposit their energy elsewhere. ${ }^{30}$ This quantity is of great importance due to its close correlation with biological damage. ${ }^{31-33}$ Typically, LET is reported using units of $\mathrm{keV} / \mu \mathrm{m}$.

\subsection{Absorbed Dose}

This chapter began by stressing the importance of being able to quantify 'amounts' of radiation and it was mentioned that 'absorbed dose' was used across all disciplines for this purpose. This quantity will be formally defined and described in this section. As explained previously, when a photon is incident upon a target, it causes the release of light charged particles through one of or a combination of the three most probable photon interactions (Compton effect, photoelectric effect, pair production). These light charged particles then go on to deposit their energies as described in Section 2.2. 
Once the energy absorbed in the medium is obtained, the absorbed dose to the medium can be obtained by normalizing the radiation energy absorbed to the mass of the volume being examined.

Under certain conditions, absorbed dose can be obtained by examining the electron fluence passing a specific volume. When the condition of charged particle equilibrium is reached, absorbed dose to the medium can simply be obtained by multiplying the electron fluence $\left(\varphi_{\text {med }}\right)$ in the medium by the mass collisional stopping power:

$$
D_{\text {med }}=\varphi_{\text {med }}\left(\frac{S_{c o l}}{\rho}\right)_{\text {med }} .
$$

Charged particle equilibrium is reached when there is enough fluence of electrons such that in a given volume, for each electron exiting there is one entering resulting in a net equilibrium of electron flux. In the cancer clinic, absorbed dose is typically measured using a device known as an 'ionization chamber' which measures the charge produced by electrons released by photons in an electric field. This measurement of charge is converted to absorbed dose by using primary national standards-traceable calibration factors obtained using water calorimetry or other primary standards.

\subsection{Relative Biological Effectiveness}

Thus far, this section has focused on describing the passage of radiation through matter in general. The main interaction possibilities for both electromagnetic and charged particles have been covered. Across the distinct disciplines that utilize 
radiation, the main quantity of interest is how much damage radiation is causing to the living cells of the body. Historically, this 'radiation damage' has been quantified by comparing the damage done by a type of radiation relative to the damage done by some reference radiation.

This quantity is known as the relative biological effectiveness, or RBE. Formally, it is defined as the amount of dose required to reach a given biological endpoint (e.g. LD50, or $10 \%$ cell survival) using a test radiation normalized to the amount of dose required to reach the same endpoint by a reference radiation (Equation 2.3, Figure 2.5). This can be seen through,

$$
R B E=\frac{D_{\text {reference }}}{D_{\text {test }}}
$$

where RBE is the relative biological effectiveness, and $D_{\text {test }}$ and $D_{\text {reference }}$ are the respective absorbed doses required to reach the chosen biological endpoint.

Typically, these studies have been conducted with the aim of better understanding how changing particle type or energy can affect the destructive impact on biological tissue. Various prokaryotic and eukaryotic cells have historically been used as the biological test medium when evaluating RBE. For example, proton RBE has been evaluated using human cervix cancer cells, V79 Chinese hamster cells, U2OS, and human laryngeal cells. ${ }^{33-36}$ 


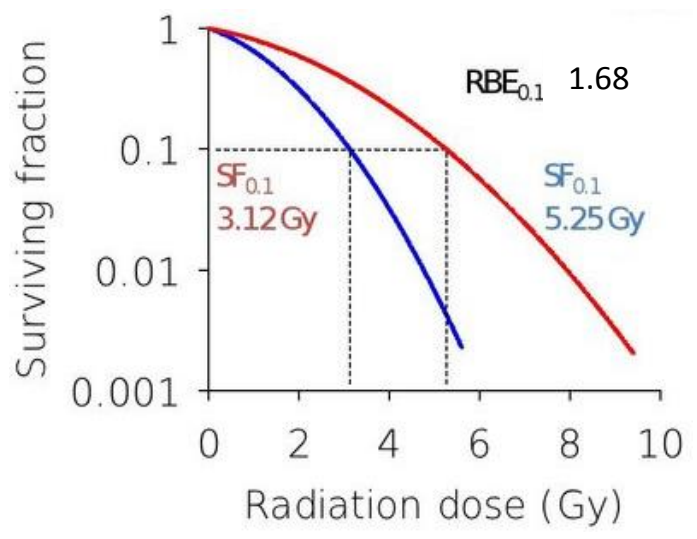

Figure 2.5: Sample calculation of neutron RBE relative to x-rays using cell survival curves. Adapted from Sorensen, B.S. ${ }^{37}$

This variation in cell killing potential is of utmost importance, especially in health physics and nuclear safety applications. Depending on particle type, the ability for radiation to cause damage to tissue can vary drastically - as an example, Howell et al, determined that the RBE of alpha particles relative to $120 \mathrm{kVp} x$ rays was 4.7 when a survival endpoint of $37 \%$ was chosen (biological model was spermatogenesis in mouse testes) ${ }^{38}$ In the event of a radiological emergency, determining the type of radiation present is of utmost importance.

There is a strong relationship between the linear energy transfer and the relative biological effectiveness. LET is the amount of energy absorbed by a medium per unit pathlength of a particle traversing through it. When that medium is biological tissue, the impact of LET variation becomes clear. It is a well-established fact that radiation damage to tissue primarily occurs via mitotic cell death. ${ }^{2}$ lonizing radiation causes accumulated damage to the DNA of cells, which causes them to lose reproductive integrity over time. 
As the linear energy transfer increases, the distance between subsequent energy deposition events decreases (Figure 2.6). This causes the likelihood of a single particle causing double stranded DNA breakages more likely. It is also a well-established fact that, while single stranded DNA breakages are easily reparable by the cell's repair mechanism, double stranded breakages pose a much greater challenge.$^{39}$ Thus, due to increased damage to the cell's DNA resulting from increased LET, a strong correlation between LET and the previously explained RBE exists (Figure 2.7).

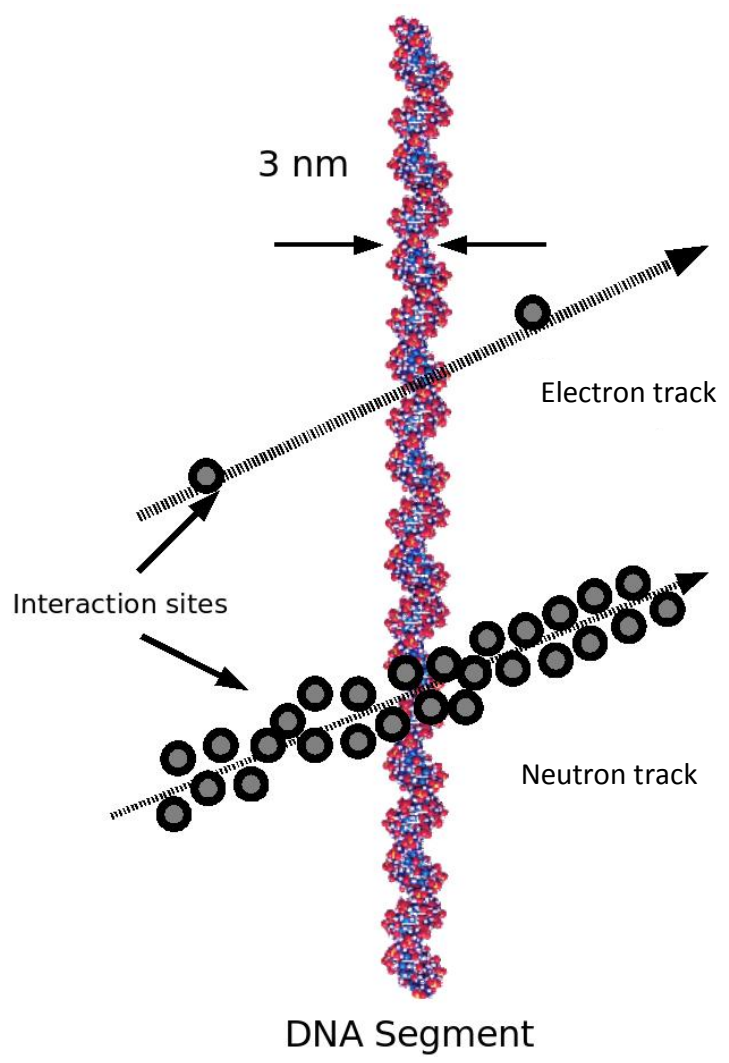

Figure 2.6: Low vs high LET radiation tracks. ${ }^{40}$ Low LET radiation, shown on the track above, is less likely to cause irreparable DNA damage to the cell. 


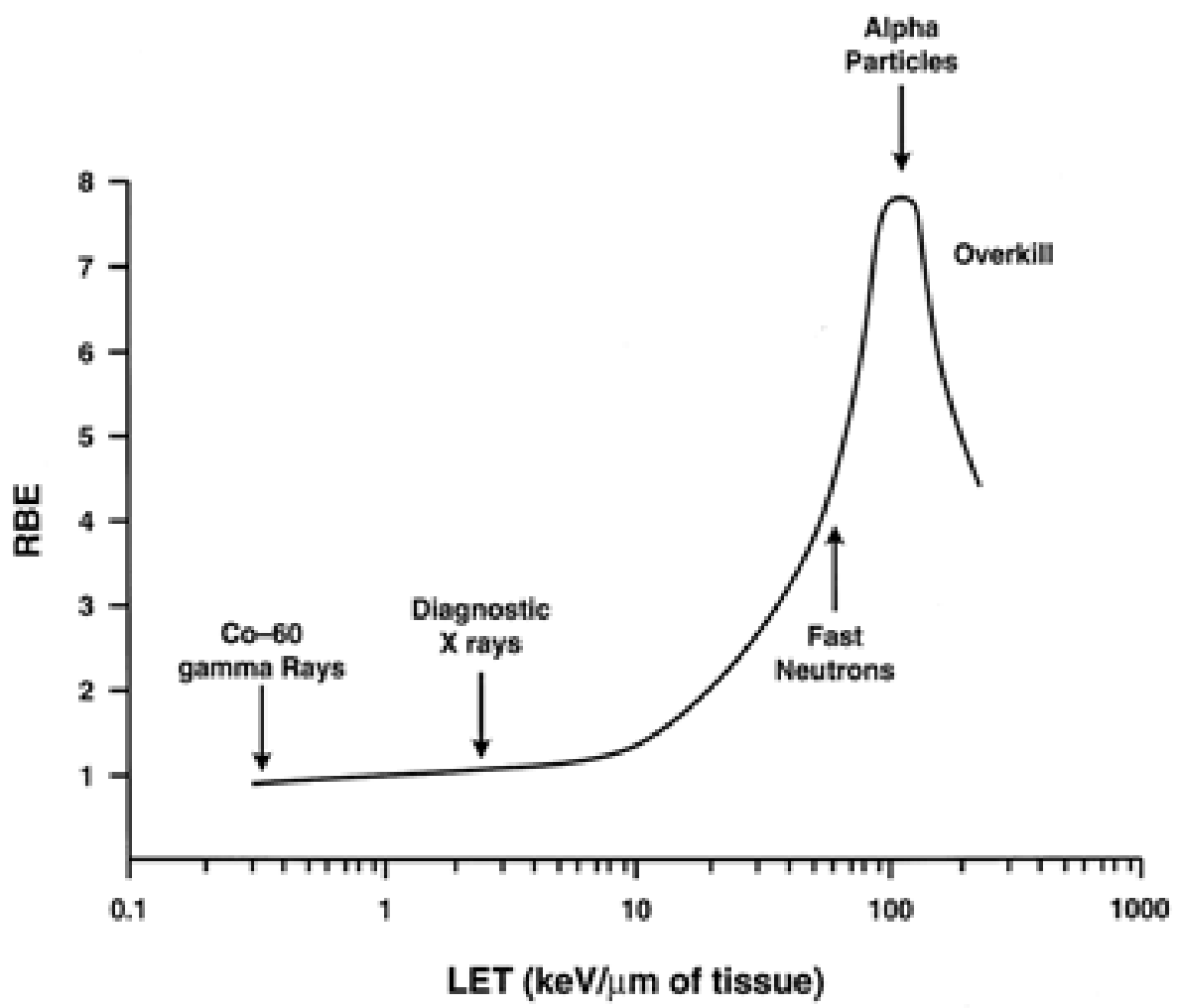

Figure 2.7: RBE shown as a function of increasing LET. The maximum RBE occurs when LET is equal to $100 \mathrm{keV} / \mu \mathrm{m}^{2}$ It is important to note that not all radiation types have an LET equal to the depicted value (e.g. diagnostic $x$ ray LET is not always 2.6 $\mathrm{keV} / \mu \mathrm{m})$.

\subsection{Monte Carlo Simulation}

The behavior of radiation travelling through matter can be modelled through complex computer simulation. Specifically, radiation transport is commonly modelled using Monte Carlo techniques. This allows for the determination of various dosimetric quantities, such as energy and dose deposition, stopping power, and range through computational techniques. Despite not being able to be solved analytically, the 
probability of interactions occurring can generally be predicted quantitatively. By accounting for the interaction probabilities of various types of radiation, the transport of each particle through a volume of matter can be simulated. By simulating large numbers of particles, each with their own associated history, trends and quantities can be extracted from the data. Monte Carlo techniques have been used computationally for decades and mathematically for centuries. ${ }^{41}$ Its use spans various fields such as finance, physics, atmospheric modelling, and traffic flow simulations to name a few examples.

The Monte Carlo method allows for the integration of a very large number of stochastic trajectories to derive useful conclusions. The first step is to generate a random number, then sample that random number to a given probability density function relevant to the stochastic process being examined, and finally use the sampled value to calculate a specific quantity (e.g. interaction distance, energy deposition, etc.). Following this, the previous steps are repeated using a new random number. In order to successfully simulate radiation transport using Monte Carlo, there are four major requirements: 1) a random number generator, 2) geometry, 3) physics processes and interactions input, and 4) result storage. ${ }^{42}$ Looking at one individual history does not provide meaningful insight into the behavior of radiation because each single step and interaction is based on a random number. Only by aggregating the results of a large number of histories can meaningful conclusions be drawn. In order to understand how many individual histories must be run, the tolerable simulation uncertainty must be determined. For example, in the simulations described in Chapter $5,10^{9}$ histories were required in order to obtain an absorbed dose uncertainty of $0.05 \%$. 
There are several different Monte Carlo codes that can be used to simulate radiation transport through matter. These include software packages such as EGSnrc, PENELOPE, GEANT4, and MCNP. ${ }^{43-46}$ In this work, GEANT4 was utilized for its breadth and customizability. GEANT4, an acronym for Geometry ANd Tracking (version 4) is a powerful Monte Carlo toolkit developed by researchers at the European Organization for Nuclear Research (CERN) to simulate radiation transport through matter. ${ }^{45}$ This software package utilizes object-oriented programming $\left(\mathrm{C}_{++}\right)$and allows for the simulation of over 100 different particle types with various energies spanning from very low to very high using several different physics packages.

In order to simulate radiation transport accurately, the world and phantom geometry must first be defined. In GEANT4, custom materials can be created by inputting atomic compositions, or one can choose from a wide selection of pre-made NIST materials to use in the simulation. Various complex geometries, shapes, and setups can be simulated. Next, the user must define the appropriate physics package for the software to use. There are several different physics lists which can be enabled if required; for example, the standard electromagnetic physics ("G4 Standard EM Physics") package is used to simulate electromagnetic interactions of charged particles, gammas, and optical photons and is valid in the energy range of $1 \mathrm{keV}$ to $10 \mathrm{PeV}$. In the case where electromagnetic interactions for particles with energy less than $1 \mathrm{keV}$ must be simulated, a new physics list can be used. Either 'G4 Low Energy EM Physics' or 'G4 DNA Physics' would be appropriate. Similarly, various physics lists for heavy particle interactions and for optical photon production (scintillation, Cherenkov light) exist. $^{47}$ 
Once the physics list has been chosen, the user must define the particle generator's beam type, shape, direction, and particle. GEANT4 can be installed and run on parallel processors, allowing for fast simulation times. The collection of various quantities of interest such as energy deposited, particle flux, and so on is referred to as 'scoring'. In GEANT4, scoring can be done through sensitive detectors or through command-based scoring. The sensitive detector approach uses the simulation geometry and shapes already defined as the surface or volume in which the dosimetric quantities of interest are scored. In command-based scoring, the user can define a voxelized shape which does not interact with the simulation geometry at all and dosimetrically interesting quantities can be scored. These include energy deposited, dose deposited, cell current, cell flux, population, and surface cell charge. In order to evaluate the statistical uncertainty on any given simulation, a voxel equal in size and purpose must be created using the main build file and the calculation for absorbed dose uncertainty, for example, can be added.

The termination of a particle's track when simulating radiation transport is determined by the energy cut-off. At each interaction, the particle dissipates some amount of energy (governed by the Monte Carlo process at each step by sampling the interaction cross-sections using the random number generator); this process could continue indefinitely as the particle's energy approaches an asymptote at zero. This would cause the simulation to crash due to a never-ending track and unachievable result. In order to avoid this, the energy threshold is used to terminate the particle's track. 
In the Monte Carlo simulation, a radiation source that emits the particles defined by the user in the direction desired must be defined. For some applications, the modelling of the radiation source can become an incredibly complex simulation in and of itself. For example, in order to simulate the radiation being emitted from a linear accelerator, all of the components between the high- $Z$ target and the patient must be modelled. This includes components such as the jaws, multi-leaf collimators, monitor ionization chamber, flattening filter, and several others. Modelling these components, as well as all of the radiation interactions occurring in them would involve a large simulation taking a very long time to yield results. Fortunately, instead of modelling all of the components in the head of the linear accelerator, a 'phase-space' file can be used as the radiation source. This file, generated by other researchers who modelled and validated all of the linear accelerator components, contains the energy, momentum, direction, and particle type for all of the radiation leaving the machine. ${ }^{48}$ This file can be used as a source itself for cases in which a complex radiation source is required, as seen in Chapter 3 , where a phase-space modelling a Varian Clinac $600 \mathrm{C}$ is used. ${ }^{22}$

\subsection{Plastic Scintillator Detectors}

Plastic scintillators are organic materials which emit light upon interacting with ionizing radiation. The scintillation mechanism in organic compounds is essentially an excitation of the electron cloud contained within the hybridized ring of the aromatic scintillator molecule. Upon interacting with ionizing radiation, the electron cloud is excited and the subsequent de-excitation results in the emission of an ultraviolet 
photon. The plastic scintillator volume contains wavelength shifters and phosphors which then interact with the newly released photon, converting it to be in the visible wavelength region (approximately $440 \mathrm{~nm}$ ). These materials are versatile and useful in radiation measurement and detection (Figure 2.8). Several commercially available devices utilize plastic scintillators - an example from the radiotherapy field is the Exradin® W1 and W2 scintillator detectors (Figure 2.9).

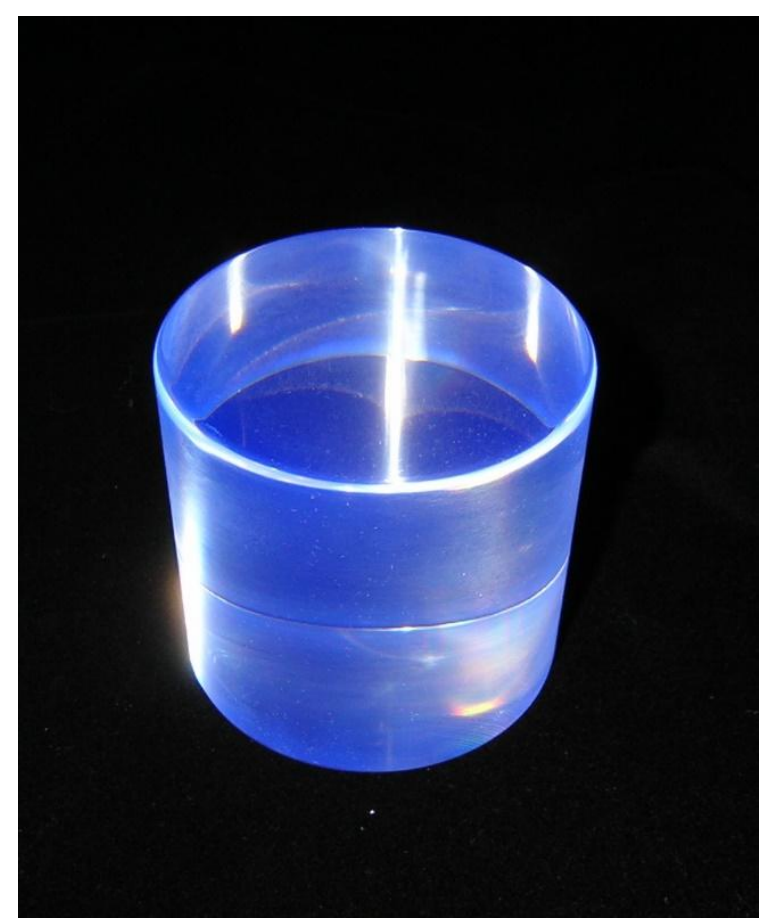

Figure 2.8: Plastic scintillator emitting its characteristic blue light (Eljen Technologies, Sweetwater TX). ${ }^{49}$ 


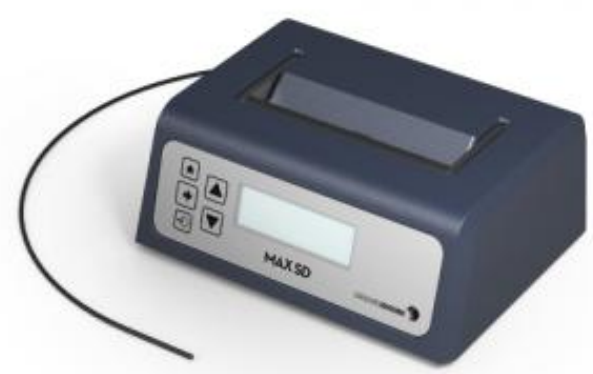

Figure 2.9: The W2 detector produced by the radiotherapy equipment company, Exradin..$^{50}$

Plastic scintillators are excellent to use for measurement because their signal can easily be read using a photodetector (photomultiplier tube, silicon photomultiplier, and so on). It has been demonstrated by Beddar et al., that these detectors exhibit linearity and energy independence above $100 \mathrm{keV}{ }^{51}$ They are also widely used for detection in particle hadronic physics as well as in health physics.

The response of plastic scintillators can be modelled using Equation 2.4

$$
\frac{d L}{d x}=L_{0} \frac{d E / d x}{1+k B \times d E / d x}
$$

where $L_{0}$ is the scintillator's light yield (photons per $\mathrm{MeV}$ radiation energy absorbed), $k B$ is the quenching parameter, and $d E / d x$ is the collisional stopping power, or LET of radiation passing through the scintillator. This equation is referred to as Birks' Law, 
discovered by J.B. Birks in the 1950s. ${ }^{52}$ Thus, it is clear that the response of plastic scintillators is intrinsically dependent upon the LET of radiation passing through it.

When exposed to high LET radiation, the scintillator exhibits a quenched response. This can be seen in Birks' law - as $d E / d x$ increases, the ratio of numerator and the denominator will eventually reach unity. Scintillator quenching is the main reason why it has not yet been widely adopted for heavy particle or low energy dosimetry.

The main detection mechanism presented in this thesis is based upon this fact. This project utilized lead doped plastic scintillators. By doping the scintillator volume with lead, a high $Z$ element, scintillator was made to be energy dependent. This is because the addition of lead causes a large change in how photons and electrons interact within the scintillator volume. By using various differently doped scintillators, the LET spectra of various radiation beams were resolved (Chapter 6). 


\section{Evaluating the Biological Impact of Increased Scattered Radiation in Single and Composite Field Radiation Beams}

This Chapter is based on an original article which has been published as:

H. Nusrat, G. Pang, S.B. Ahmad, A. Sarfehnia

"Evaluating the biological impact of increased scattered radiation in single and composite field radiation beams". (2018)

Biomedical Physics and Engineering Express, 4(3), DOI: 10.1088/2057-1976/aab0db

\subsection{Abstract}

Scattered radiation seen at the edges of a treatment field consists of low energy electrons and photons. Lower energy particles have a higher LET, making them more biologically damaging. With a single beam of a large field-size (FS), the amount of these highly damaging electrons is low relative to within the treatment beam. However, when multiple beam angles and smaller FS are used (as in IMRT/VMAT), the relative contribution of scattered radiation increases and biological consequences may vary. The purpose of this study was to evaluate the theoretical impact of increased scattered radiation in single and composite field beams. Monte Carlo Geant4.10.3 was used to 
score the electron energy spectra at different depths within a water phantom with a 6 MV photon beam incident. The energy spectra were then used to calculate the maximum RBE, $\mathrm{RBE}_{\mathrm{M}}$. Beams examined were phase-spaces of a Varian Clinac $600 \mathrm{C} 6$ MV linac $\left(10 \times 10 \mathrm{~cm}^{2}\right.$ beam, $1 \times 1 \mathrm{~cm}^{2}$ beam), and a composite $10 \times 10 \mathrm{~cm}^{2}$ beam. The composite $10 \times 10 \mathrm{~cm}^{2}$ treatment field (simple IMRT) was created by summating one hundred $1 \times 1 \mathrm{~cm}^{2}$ beams to form a relatively uniform $10 \times 10 \mathrm{~cm}^{2}$ field. For smaller FS (1.5 cm depth), an increase in $\mathrm{RBE}_{\mathrm{M}}$ was seen $5.2 \mathrm{~cm}$ outside the beam (17\%). The $10 \times 10 \mathrm{~cm}^{2}$ beam showed an increase of $14 \%, 9.2 \mathrm{~cm}$ away from beam's edge $(1.5 \mathrm{~cm}$ depth). The composite $10 \times 10 \mathrm{~cm}^{2}$ beam exhibited similar $\mathrm{RBE}_{\mathrm{M}}$ enhancement to the

$10 \times 10 \mathrm{~cm}^{2}$ phase-space, however, the region of increased damage occurred closer to the beam ( $5.6 \mathrm{~cm}$ away). The results indicate that although the region inside the primary beam is not affected, the contribution of damaging particles happens much closer to the beam's edge in the composite field case relative to an open field. This may have potential implications regarding the effective dose to organs at risk during radiotherapy.

Keywords: RBE, modulated beams, out-of-field dosimetry, scattered radiation

\subsection{Introduction}

Collimation of radiation beams used in cancer radiotherapy is required in order to limit the beam's field size to be large enough to irradiate the tumour while minimizing exposure to healthy tissue. In modern linear accelerators (LINACs), collimators and jaws composed of high atomic number elements are used in order to block undesired regions of the beam. Interactions occurring at the edges and lateral faces of these 
collimators can result in the production of secondary radiation, commonly referred to as head scatter. ${ }^{53}$ This scattered radiation consists primarily of low energy photons and electrons, which are typically of a higher linear energy transfer (LET), and therefore more biologically damaging. ${ }^{54}$

When the simple case of a conventional radiotherapy treatment (Figure 3.1) is considered, the amount of high-LET, highly damaging scatter produced is low relative to the primary beam. Previous studies have attempted to determine whether or not any change occurs at the edge of a radiation field in terms of LET or RBE (relative biological effectiveness). Kirkby et al. (2007), ${ }^{56}$ conducted a Monte Carlo study using various clinical radiation beam models and found an increase in the average LET, suggesting an increase in RBE outside the treatment field for a single beam. Liu and Verhagen (2002), ${ }^{57}$ demonstrated a $20 \%$ increase in radiation quality factor, $Q$, in the penumbra when compared to the central axis in another Monte Carlo study. Another group attempted to determine any change in ' $Q$ ' in a TomoTherapy® dose distribution using Monte Carlo simulation and found a $1.5 \%$ increase at certain positions. ${ }^{58}$ These previous works have attempted to explore the question of radiobiological variability outside the treatment beam, but have been limited by looking at radiobiological changes at a limited number of points. Other groups have conducted experiments in which cell survival was examined in and out of the radiation field; the results are in agreement that cell survival outside of the field was lower than previously expected. Butterworth et al. (2011), ${ }^{59}$ conducted an experiment in which cell survival was examined within and out of the radiation field; they found that cell-killing in the out-of-field region was significantly higher. Trainor and colleagues ${ }^{60,61}$ also examined cell survival outside of the primary 
beam using various cell lines and observed increased DNA damage in the out-of-field regions. The studies conducted by Trainor et al., attributed the increased DNA damage in out-of-field regions partly to changes in intercellular communication in mediating cellular response. McGarry et al. (2012), ${ }^{62}$ conducted a similar study and found that outof-field survival was independent of cell line, delivery technique, and dose; however, a statistically significant difference was observed in cell survival in field versus out-of-field, only when intercellular communication was kept intact.

It is also important to mention the work conducted by Chofor et al. (2012), ${ }^{63}$ which studied only the internal scatter component of the peripheral dose; it was found that dose to the near periphery is comprised mainly of internal scatter, while the far periphery is mainly head scattering. Ezzati et al., ${ }^{64}$ found that no significant change in RBE occurs when comparing the in-field region to the out-of-field region. They conducted a Monte Carlo study in which DNA double stranded breaks were scored and RBE calculated. In contradiction to this, Okamoto et al. (2011), ${ }^{65}$ measured the lineal energy transfer distributions for $200 \mathrm{kV}$ x-rays and megavoltage photon beams; they found that the lineal energy of the low energy $x$-rays was significantly greater than that of the $6 \mathrm{MV}$ x-rays, yielding a higher RBE.

The disagreement among previous studies and the lack of literature on composite and modulated radiation fields that better approximate current treatment methods warrants further investigation.

In order to examine the damaging effects of radiation at different points, the spatial variation in the 'maximum RBE', or $\mathrm{RBE}_{\mathrm{M}}$ was used. As demonstrated by Dale and Jones, ${ }^{66}$ at the low dose limit, the $\alpha / \beta$ ratio becomes equivalent to the maximum 
relative biological effectiveness $\left(\mathrm{RBE}_{\mathrm{M}}\right)$; this formalism is further explained in the Materials and Methods section. Briefly, the $\alpha / \beta$ ratio, another commonly used radiobiological quantity, is the point at which the linear and quadratic modes of cellkilling are equivalent.

In order to better conform the radiation dose distribution to the tumour, modern radiotherapy treatment plans typically are comprised of many small beamlets $\left(<1 \mathrm{~cm}^{2}\right.$ field size) that are often incident on the patient from multiple angles. In this case, the amount of additional higher-LET scatter due to the larger number of beamlets may accumulate and produce a summative effect in unintended locations.

The purposes of this work are twofold: First, the intent is to introduce a Monte Carlo based formalism to better evaluate the relative in-field and out-of-field radiation damage by calculating spatial $\mathrm{RBE}_{\mathrm{M}}$ distribution. With the concept of $\mathrm{RBE}_{\mathrm{M}}$ as a proxy to radiobiological damage, we will then study the relative radiobiological damage differences between in-field and out-of-field for single and composite radiotherapy fields. 


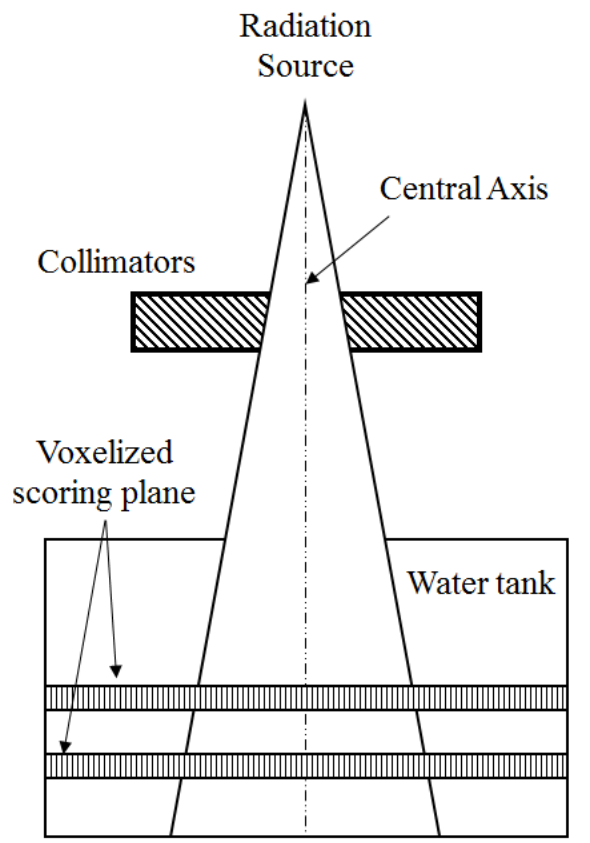

Figure 3.1: Schematic of radiation source incident upon a water phantom. Scoring planes placed at two depths for each beam, electron energy spectra were scored at all positions in the plane.

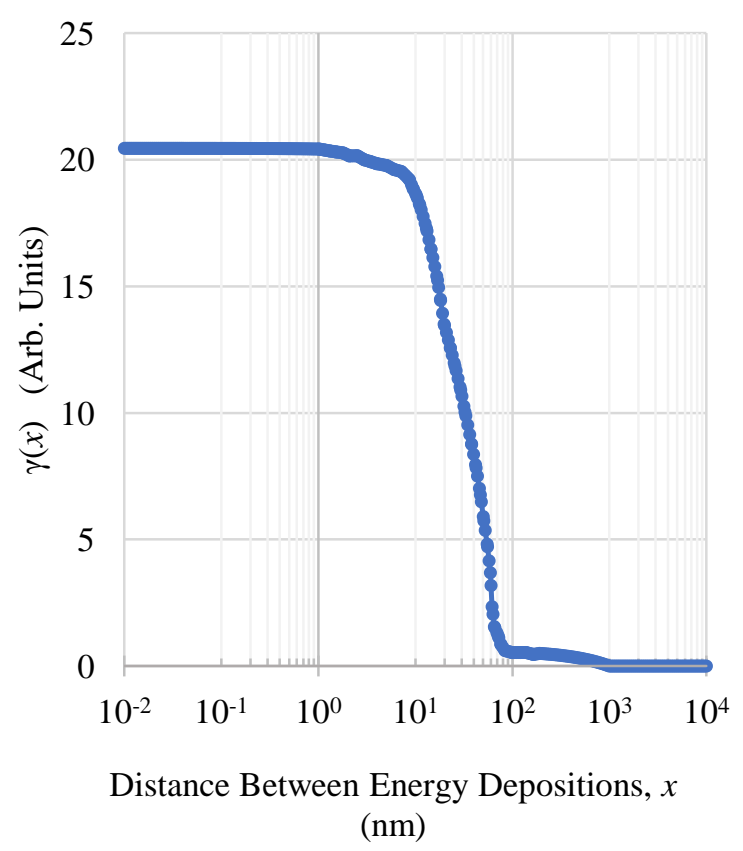

Figure 3.2: The $y$ parameter as a function of $x$. This quantity represents the probability of a lesion occurring given that two energy depositions occur some distance $(\mathrm{x})$ apart for a given electron. ${ }^{67}$

\subsection{Materials and Methods}

\subsection{1. $\overline{\alpha / \beta}$ Ratio Formalism}

In order to quantify radiobiological significance, the $\alpha / \beta$ ratio was used. The $\alpha / \beta$ ratio represents the dose level at which the amount of cells killed by double stranded and single stranded DNA breakages are equivalent (in the linear-quadratic model). According to the theory of dual radiation action, ${ }^{68}$ the $\alpha / \beta$ ratio for monoenergetic particles (electrons) is given by 


$$
\frac{\alpha}{\beta}(E)=\int_{0}^{\infty} t(x, E) \gamma(x) d x,
$$

where $t(x, E)$ is the proximity function, which is the probability that an electron of energy, $E$, undergoes two energy depositions, a distance $x$ apart. Proximity functions in water for electrons from $100 \mathrm{eV}$ to $10 \mathrm{MeV}$ are given by Chen and Kellerer (2007). ${ }^{69}$ The gamma function, $\mathrm{Y}(x)$, is the probability that two energy depositions, a distance $x$ apart, result in a lesion. The quantity $\gamma(x)$ is only dependent on the biological system (not on the radiation used) and is non-zero only when $x$ is small. The gamma function used in this work was representing lesion probability in V79 Chinese hamster cells in late-S phase. $^{67}$

For a more realistic scenario, the weighted $\alpha / \beta$ ratio for a polyenergetic electron energy spectrum (henceforth represented as $\overline{\alpha / \beta}$ ) can be determined by,

$$
\overline{\alpha / \beta}=\frac{\int_{0}^{\infty} \frac{\alpha}{\beta}(E) n(E) E d E}{\int_{0}^{\infty} n(E) E d E},
$$

where $\alpha / \beta$ is the $\alpha / \beta$ ratio calculated for the monoenergetic electron energy $E$ using Eq. 3.1 , and $n(E)$ represents the electron energy spectrum, with $E$ representing discrete energy bins. At the low-dose limit, the $\overline{\alpha / \beta}$ becomes the maximum RBE, $\mathrm{RBE}_{\mathrm{M}}{ }^{66}$

\subsubsection{Obtaining $n(E)$, the Electron Energy Spectra}

The electron energy spectra were obtained using Geant4.10.3 Monte Carlo simulations. In the simulation, various different linear accelerator beams were set 
incident upon a water phantom. Using Geant4, a voxelized scoring plane spanning the size of the water phantom (at a given depth) was used to determine the electron spectra present in different regions (Figure 3.1). Electrons were first scored and then placed into various energy bins which ranged in size from $920 \mathrm{eV}$ to $1.6 \mathrm{MeV}$. For each beam, three types of scoring orientations were used: two different depths (depending on the type of beam), and one depth profile plane.

In order to include scattered radiation in the Monte Carlo simulation, LINAC phase-space files were used. A phase-space is a collection of the energy, particle type, position, direction, progeny, and statistical weight of particles generated by a radiation source; the phase-space files used in this work were obtained from the I.A.E.A Nuclear Data Services. ${ }^{70}$ The radiation phase-spaces used in this work included: the Varian Clinac 600C $6 \mathrm{MV}$ (photon) $10 \times 10 \mathrm{~cm}^{2}$ and the $1 \times 1 \mathrm{~cm}^{2}$ beam. For each radiation treatment beam, the electron energy spectrum was scored at all positions at two depths $(1.5 \mathrm{~cm}$ and $5 \mathrm{~cm}$ in water), and the $\overline{\alpha / \beta}$ ratio was examined as distance from the beam's central axis (CAX) was increased.

For each case, the $\overline{\alpha / \beta}$ ratio was calculated at all positions in the water phantom, yielding a two-dimensional distribution at a given depth. In order to show these results, a single strip spanning the center of the phantom was extracted and the corresponding $R_{B E}$ values were displayed. The $R B E_{M}$ values were obtained for a given beam by normalizing the $\overline{\alpha / \beta}$ ratio to the $\overline{\alpha / \beta}$ on the central axis. Monte Carlo simulations were ran for $2^{31}$ initial particles, yielding an absorbed dose uncertainty better than $0.1 \%$ inside the field, using the same voxels used to score $n(E)$. 


\subsubsection{Composite $10 \times 10 \mathrm{~cm}^{2}$ Field}

The radiobiological implications (via $\mathrm{RBE}_{\mathrm{M}}$ ) of creating a larger composite field size from multiple smaller beamlets was examined by using the electron energy spectra distribution created by the $1 \times 1 \mathrm{~cm}^{2} 6 \mathrm{MV}$ photon beam and conducting mathematical summation of the spectra to create a composite $10 \times 10 \mathrm{~cm}^{2}$ field. This summation was done using MATLAB R2016b (Figure 3.3) for the fluence at $5 \mathrm{~cm}$ depth.

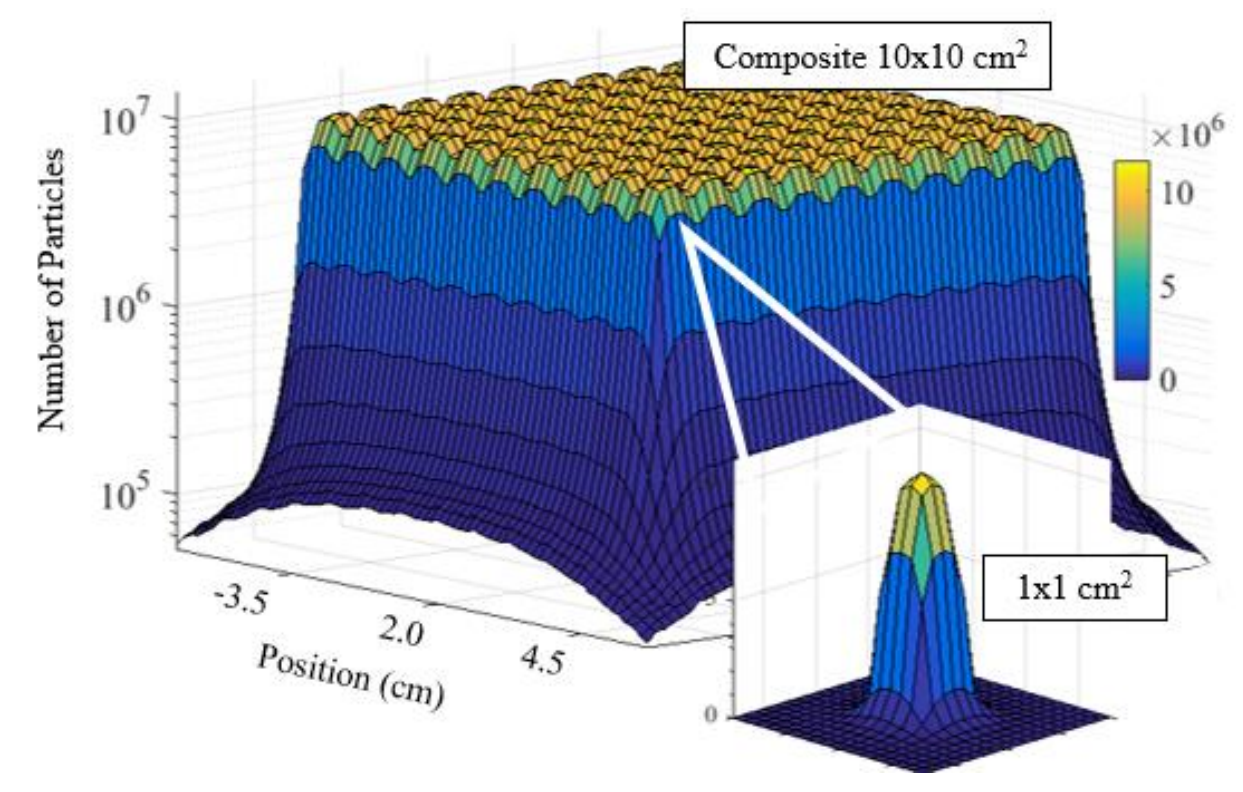

Figure 3.3: Summation of the energy spectra generated by the $1 \times 1 \mathrm{~cm}^{2}$ beam to produce a composite $10 \times 10 \mathrm{~cm}^{2}$ field size

\subsection{Results}

The radiobiological changes as a function of position for various beams are shown in Figures 3.4 and 3.5. In all figures, abscissa shows the distance away from the 
central axis $(C A X)$ of the beam, whereas the ordinate shows the $\mathrm{RBE}_{\mathrm{M}}$ (average weighted $\overline{\alpha / \beta}$ normalized to the same quantity at CAX). Figure 3.4A depicts the damage enhancement at two depths $(1.5$ and $5 \mathrm{~cm})$ for the $10 \times 10 \mathrm{~cm}^{2} 6 \mathrm{MV}$ photon beam. Figure $3.4 \mathrm{~B}$ shows the same for the $1 \times 1 \mathrm{~cm}^{2}$ beam and Figure $3.4 \mathrm{C}$ portrays the inplane versus cross-plane radiobiological variability for a $10 \times 10 \mathrm{~cm}^{2}$ beam at a depth of $5 \mathrm{~cm}$. The radiobiological variation in the composite large field compared to a single beam large field size is shown in Figure 3.4D. Figure 3.5 shows the radiobiological variation of the composite and single fields overlaid with their respective electron fluence profiles.
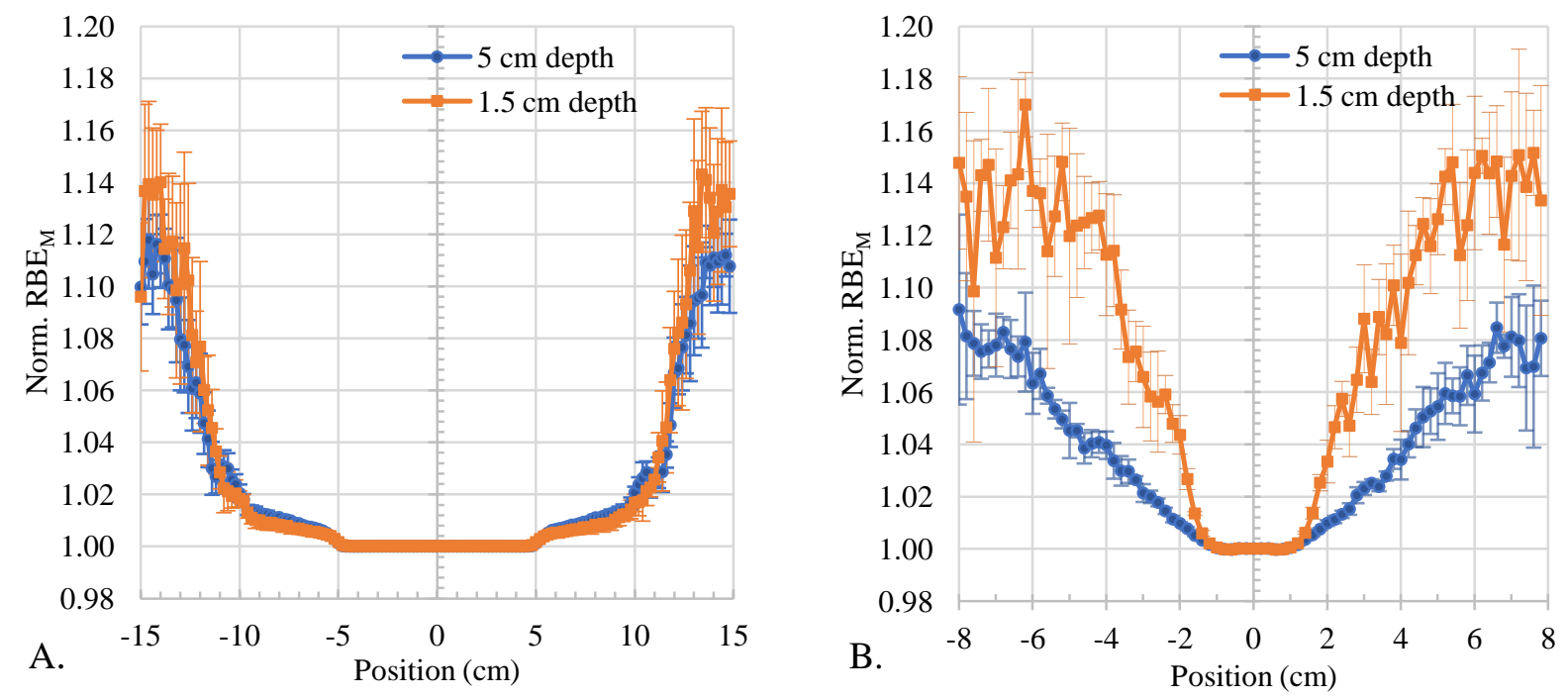

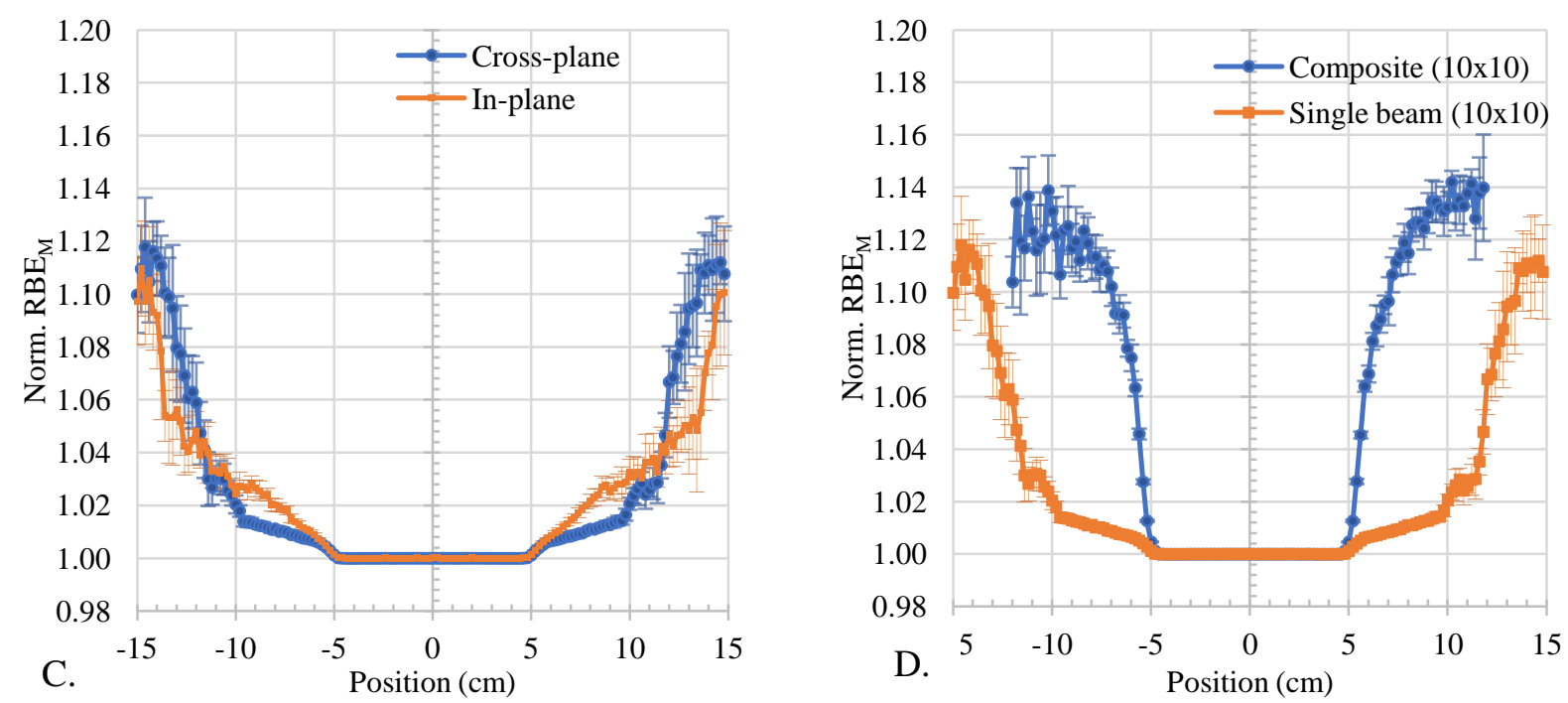

Figure 3.4: Variation in $\mathrm{RBE}_{\mathrm{M}}$ at various positions surrounding a $10 \times 10 \mathrm{~cm}^{2}(\mathrm{~A})$ and $1 \times 1 \mathrm{~cm}^{2}$ (B) $6 \mathrm{MV}$ beam. The $\overline{\alpha / \beta}$ calculated for each voxel was normalized to the central axis $\overline{\alpha / \beta}$. Variability curves are shown at depths of $1.5 \mathrm{~cm}$ and $5 \mathrm{~cm}$. The $\mathrm{RBE}_{\mathrm{M}}$ profile in the in-plane and cross-plane direction for $10 \times 10 \mathrm{~cm}^{2}$ (C). Comparison of $\mathrm{RBE}_{M}$ variation in the composite $10 \times 10 \mathrm{~cm}^{2}$ field and the single $10 \times 10 \mathrm{~cm}^{2}$ field at a depth of $1.5 \mathrm{~cm}(\mathrm{D})$. 


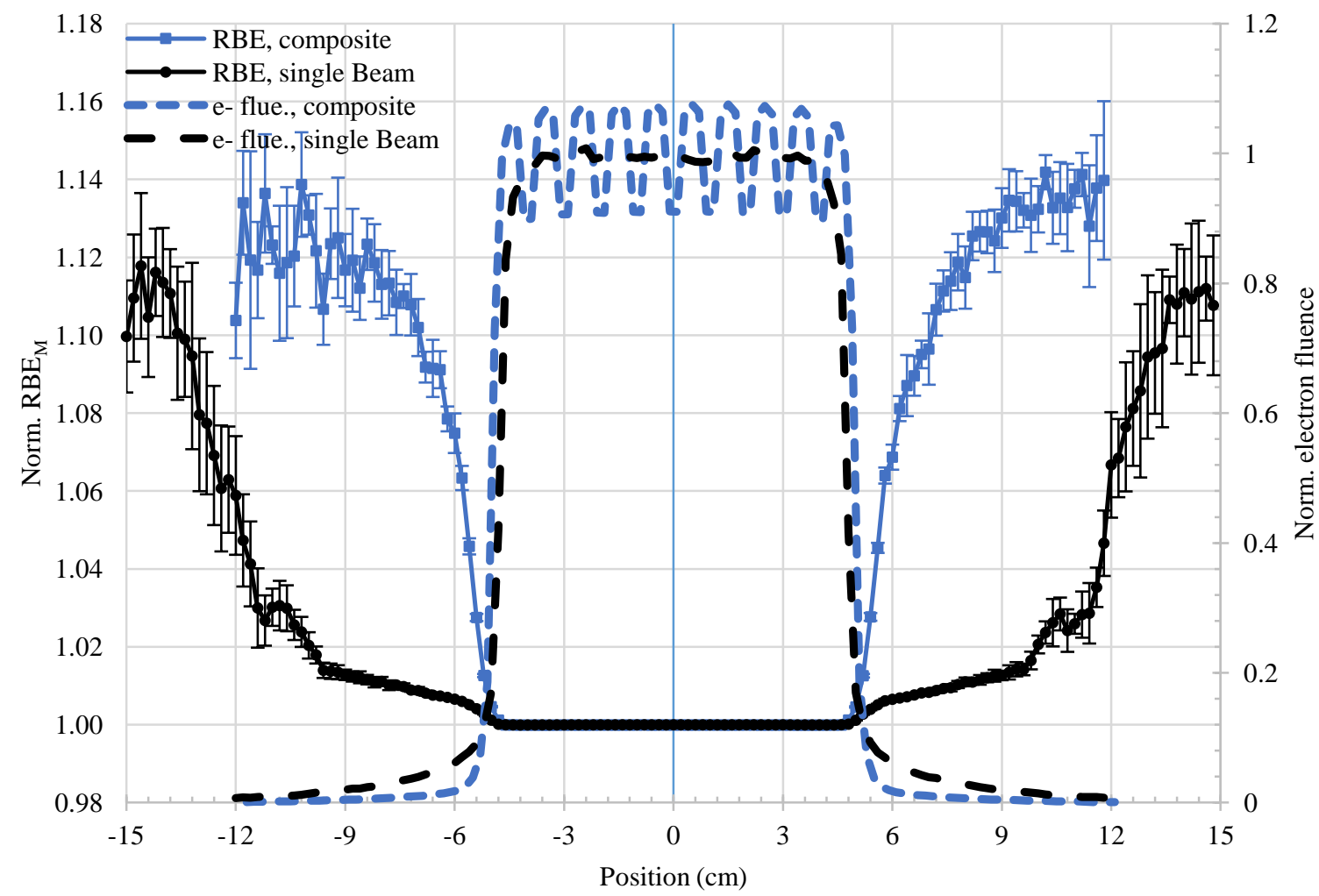

Figure 3.5: Normalized $\mathrm{RBE}_{\mathrm{M}}$ values for the composite and single $10 \times 10 \mathrm{~cm}^{2}$ plotted along with their corresponding electron fluence at various positions at a depth of $5 \mathrm{~cm}$. The dashed lines represent the normalized electron fluence (all energies) present in both cases (blue: composite field, black: single beam). The markers represent the normalized $\mathrm{RBE}_{\mathrm{M}}$ for both cases (blue: composite field, black: single beam).

\subsection{Discussion}

This work focuses on examining the spatial variation in radiobiological effectiveness of absorbed radiation dose for a $6 \mathrm{MV}$ treatment beam. Current treatment planning techniques make the assumption that low energy scattered radiation at the edges of a treatment beam has identical biological impact as the high energy primary beam in the field. Given that in conventional 3D radiotherapy treatment plans, the total 
number of these scattered, highly damaging particles is relatively low (compared to primary particles within the beam), this assumption is valid. ${ }^{58}$ However, as radiation therapy treatments move towards becoming increasingly modulated, the amount of scattered radiation present with respect to the overall primary increases. For example, in an IMRT or VMAT treatment, there are numerous small aperture radiation beamlets incoming from many different angles. This causes the scattered particles in the penumbral and out-of-field regions to accumulate, and the assumption that any increased damage they cause is negligible may no longer hold.

The $\mathrm{RBE}_{\mathrm{M}}$ values presented in Figures 4 and 5 were generated by normalizing all $\overline{\alpha / \beta}$ ratios to the $\overline{\alpha / \beta}$ on the central axis. The data in Figures 4 and 5 were presented as $\mathrm{RBE}_{\mathrm{M}}$ since according to the work done by Dale and Jones (1999), ${ }^{66}$ the $\overline{\alpha / \beta}$ ratio becomes $\mathrm{RBE}_{\mathrm{M}}$ at the low dose limit, which is the maximum relative biological effectiveness at a point. It is important to note that an electron energy cut-off of $990 \mathrm{eV}$ was used in this work; any electrons below this threshold were not transported and therefore the results seen at the lower energy bins are in fact underestimating the actual number of very low energy electrons. In the calculation of $\overline{\alpha / \beta}$, the $\mathrm{y}$ function (Figure 3.2) was extrapolated to an energy deposition distance of $0 \mathrm{~nm}$. This assumption was deemed fair given that the gamma function does not change significantly as energy deposition distance approaches zero (Figure 2). The proximity function data from Chen and Kellerer (2007), ${ }^{69}$ had a lower limit of $100 \mathrm{~nm}$.

Examining the $10 \times 10 \mathrm{~cm}^{2} 6 \mathrm{MV}$ beam revealed that the damage enhancement increases rapidly $5 \mathrm{~cm}$ past the edge of the beam, reaching a peak of $14 \%$ at $1.5 \mathrm{~cm}$ depth and $12 \%$ at a depth of $5 \mathrm{~cm}$ (Figure 3.4A). For the $1 \times 1 \mathrm{~cm}^{2}$ beam (Figure 3.4B), 
the damage enhancement effect was up to $15 \%$ at a depth of $1.5 \mathrm{~cm}, 5 \mathrm{~cm}$ away from the beam's edge ( $8 \%$ at $5 \mathrm{~cm}$ depth). Of course, the cause for the scattered radiation outside the penumbral region can also be attributed to the in-patient scattered radiation as well. It is also noteworthy that the distance (away from the edge) required before damage enhancement reached its maximum was less with the smaller field size $(3 \mathrm{~cm})$ when compared to the $10 \times 10 \mathrm{~cm}^{2}$ beam $(8.5 \mathrm{~cm})$. The $\mathrm{RBE}_{\mathrm{M}}$ distribution for the $1 \times 1$ $\mathrm{cm}^{2}$ field (Figure 3.4B) appears to be larger than the actual field-size due to the range of scattered electrons being larger than the field size, allowing for these electrons to travel beyond the field's edges. When examining any differences in radiobiological variability in the in-plane direction versus the cross-plane direction, it was found that the in-plane direction saw damage enhancement closer to the penumbral region (Figure 3.4C). This can be explained by the fact that the Varian Clinac $600 \mathrm{C}$ has its $\mathrm{Y}$-jaws above its $\mathrm{X}$ jaws, causing a slight difference in scatter conditions. ${ }^{70}$ The phase-space files used in this study were limited to jaw defined fields in order to represent a simplistic, conservative scenario.

In Figure 3.5, the electron fluence was normalized to the number of electrons on the central axis of the composite field. Although normally a composite/modulated field would be expected to have larger out of field scattered radiation than an equivalent open field, given our simplified methodology of establishing the composite field, we observed the electron fluence just outside the beam edges to be larger for the single open $10 \times 10 \mathrm{~cm}^{2}$ beam compared to an equivalent composite. There were several reasons for this; in this work, the $10 \times 10 \mathrm{~cm}^{2}$ composite was generated by summating one hundred $1 \times 1 \mathrm{~cm}^{2}$ fields separated by half their field width. As such, the composite 
was composed of beamlets that had their source position at different locations while the collimation did not change (i.e. a symmetric $1 \times 1 \mathrm{~cm}^{2}$ centered on the central axis). In reality, in external beam delivery, a composite field is generated by segments that have a fixed source position but are modulated using moving jaws and multi-leaf (MLC) collimators (i.e. off axis $1 \times 1 \mathrm{~cm}^{2}$ fields are also used). As such, the jaw-defined $1 \times 1 \mathrm{~cm}^{2}$ segments used in this work to create the composite have a much sharper penumbra and potentially less scattered radiation past their edges. In reality the segments are usually created using MLCs that can also have large geometric penumbra if they were created off-axis. Also, the uncertainties caused by the relatively low amount of particles present in the out-of-field region were propagated in the composite field summation. However, the authors would like to stress that the actual result of the exact electron fluence between single versus the composite field shown in Figure 3.5 is not as important as the observation that the out-of-field $\mathrm{RBE}_{\mathrm{M}}$ can be vastly different depending on how the field itself was created.

As discussed throughout the text, there are a few sources of uncertainty associated with this work. These can be broadly attributed to the uncertainties in the Monte Carlo calculation and the $\mathrm{RBE}_{\mathrm{M}}$ modeling. The uncertainties associated with the Monte Carlo simulation increase as the distance from the beam's primary field is increased due to the presence of fewer particles in the scoring volume as well as the low energy of those particles. ${ }^{71,72}$ A study conducted by Kirkby et al. (2007) ${ }^{56}$ examining the change in the electron energy spectra outside the beam for various radiation beams was used to validate the Monte Carlo model used in this work. In order to better quantify the uncertainty of out-of-field Monte Carlo calculations (especially due to the rapidly 
dropping number of scored particles for voxels further away from beam's edge, Figure 3.5), the same simulation was repeated ten times with different starting random seeds. All simulations were ran for $2^{31}$ particles resulting in an uncertainty on absorbed dose inside the field of better than $0.1 \%$. The error bars shown in figures 3.4 and 3.5 basically indicate the standard deviation between the $\mathrm{RBE}_{\mathrm{M}}$ results of the ten simulations for a given position in space. In the worst-case scenario, we observed a standard deviation $(\mathrm{k}=1)$ of $11.5 \%$ a distance of $6.6 \mathrm{~cm}$ away from the edge of the beam (Figure 3.4B). In Figure 3.5, the uncertainty associated with the electron fluence is not shown for clarity. As noted above, many of the low energy electrons have energies below the Monte Carlo transport cut-off of $990 \mathrm{eV}$ and are therefore not transported (the energy is deposited locally); this means that any future progeny resulting from the transport of those low energy electrons are also not created. Any potential effects of this approximation would only lead to a greater observed increase in the $\mathrm{RBE}_{\mathrm{M}}$ outside the field. Similar cut-offs as those used in our work have been used for similar type of calculations. ${ }^{55}$ Although lower cut-off values would have been advantageous, the uncertainty of the transport algorithm using our code for extremely low energies would become questionable and has not been validated. There are also uncertainties associated with the $\overline{\alpha / \beta}$ model calculation. ${ }^{67}$ As discussed above, the $\mathrm{Y}(x)$ data from Zaider and Rossi $(1988)^{67}$ is only valid for V79 Chinese Hamster cells and was extrapolated to an energy deposition distance $(x)$ of zero (a fair assumption, as discussed more in depth above). Further work to quantify the uncertainties and establish cases where the effect is relevant is underway. 
The potential clinical impact of these findings may be significant, especially in cases where the organ at risk is near the target in a radiation therapy plan. Given that the radiobiological enhancement may reach up to $17 \%$, this means that if the organ at risk is in the out-of-field region, the effective dose is also increased by up to $17 \%$. Figure 3.6 shows a schematic diagram of a spine stereotactic body radiotherapy (SBRT) treatment, where the gross disease in the spine is to be treated without overdosing the thecal sac, cord, or sacral plexus tolerance doses (the latter structures often constitute important OARs in these complicated treatments). Currently, the radiation enhancement of the out-of-field absorbed dose is neglected, and the treatment planning is performed on an absorbed dose optimization basis solely. Based on the results of this study, we note that the enhancement effect of out-of-field doses is larger than the in-field, and it is a function of the way the field was constructed. As we move towards higher localized tumor doses in ablative therapies such as the example introduced above, an accurate knowledge of the out-of-field doses to OARs may become more important. By calculating and applying the dose enhancement factor in the out-of-field region for the specific treatment plans created, we can better estimate the dose to organs at risk that may be nearby. This allows for better comparison between various treatment plans that may have been created with different segment arrangements. Moreover, given that OAR tolerance doses are often the limiting factor preventing target dose escalation, a better estimation of OAR dose may facilitate safe dose escalation in SBRT in the future. 


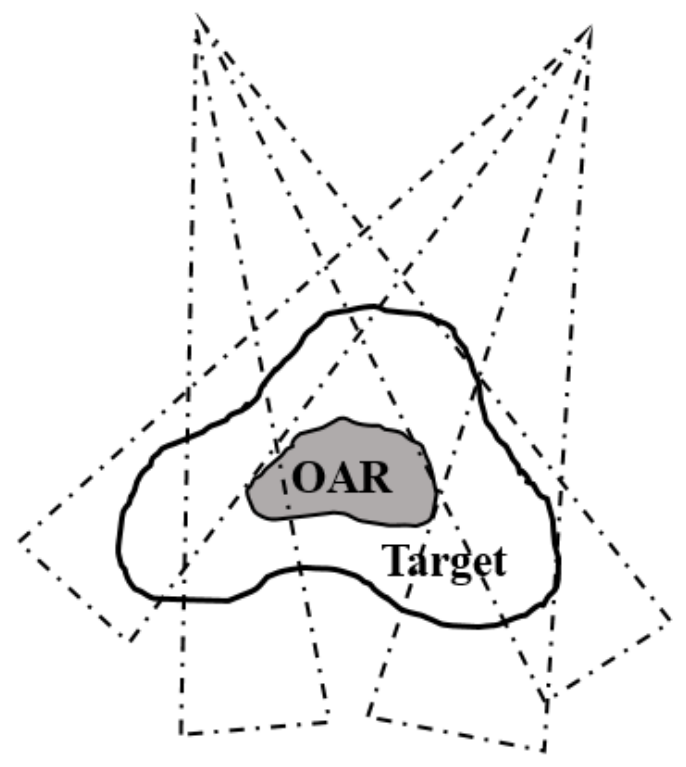

Figure 3.6: Taking into account the findings of this work, the effective dose to the organ at risk, particularly in cases where the organ at risk is in close proximity to the tumour, may be larger than anticipated, and may become significant especially if the OAR dose is pushed to near-tolerance. In the schematic diagram above such a case is shown although commonly a much greater number of beams are used.

\subsection{Conclusions}

In this work, we numerically studied the change in the electron energy spectrum over a large profile extending from the center of a radiation beam's field size all the way to large distances outside the beam's edge. It was found that the change in $\mathrm{RBE}_{\mathrm{M}}$ is heavily dependent upon how the field was created. Further studies will include the modelling of a linear accelerator head (instead of using the phase-space) to better estimate differences between realistic modulated treatment plans. This work represents 
the conservative case of $\mathrm{RBE}_{\mathrm{M}}$ variation; changes such as using more realistically collimated fields will magnify the variation observed. Possible impacts of this finding are that the effective dose to organs at risk and other tissues exposed during radiotherapy may be greater than anticipated in cases where many small beamlets and beam angles are used. Further studies to evaluate the significance of this effect in highly modulated IMRT/VMAT plan, as well as other radiation delivery modalities such as GammaKnife ${ }^{\circledR}$, different particle types, and brachytherapy treatments are currently underway.

\subsection{Acknowledgements}

This work has been supported in part by a Discovery grant of the Natural Sciences and Engineering Research Council (NSERC; Grant No. RGPIN-435608). 


\section{Maximum RBE change in ${ }^{192} \mid \mathrm{Ir},{ }^{125} \mathrm{I}$, and ${ }^{169} \mathrm{Yb}$ brachytherapy and the corresponding effect on treatment planning}

This Chapter is based on an original article which has been submitted for publication as:

H. Nusrat, S, Karim-Picco, G. Pang, M. Paudel, A. Sarfehnia

"Maximum RBE change in 192Ir, 125I, and 169Yb brachytherapy and the corresponding effect on treatment planning".

Physics in Medicine and Biology, Submitted for publication, July 2019.

\subsection{Abstract}

Purpose: The purpose of this study was to examine RBE variation as a function of distance from the radioactive source, and the potential impact of this variation on a realistic prostate brachytherapy treatment plan. Methods: Three brachytherapy sources $\left({ }^{125} \mathrm{I},{ }^{192} \mathrm{Ir}\right.$, and $\left.{ }^{169} \mathrm{Yb}\right)$ were modelled in Geant4 Monte Carlo code, and the resulting electron energy spectrum in water in 3D space around these sources was scored (voxel size of $2 \mathrm{~mm}^{3}$ ). With this energy spectrum, microdosimetric techniques were used to calculate the maximum RBE, $R B E_{M}$, as a function of distance from the source. $R_{B} E_{M}$ of ${ }^{125}$ I relative to ${ }^{192}$ Ir was calculated in order to validate simulations against literature; all 
other $\mathrm{RBE}_{\mathrm{M}}$ calculations were done by normalizing electron fluence at various distances to the source position. In order to examine the impact of $\mathrm{RBE}_{M}$ variation in treatment planning, a realistic ${ }^{192}$ Ir prostate plan was re-evaluated in terms of RBE instead of absorbed dose. Results: The $\mathrm{RBE}_{\mathrm{M}}$ of ${ }^{125} \mathrm{I},{ }^{192} \mathrm{Ir}$, and ${ }^{169} \mathrm{Yb}$ at $8 \mathrm{~cm}$ away from the source was $0.994(+/-0.002), 1.030(+/-0.003)$, and $1.066(+/-0.008)$, respectively. $\mathrm{RBE}_{\mathrm{M}}$ in the HDR prostate treatment plan exhibited several hot $\left(+3.6 \%\right.$ in $\left.R_{B E}\right)$ spots. Conclusions: The large increase $\mathrm{RBE}_{\mathrm{M}}$ observed in ${ }^{169} \mathrm{Yb}$ has not yet been described in the literature. Despite the presence of radiobiological hotspots in the HDR treatment, these variations are likely nominal and clinically insignificant.

Key Words: RBE, brachytherapy, microdosimetry, prostate brachytherapy

\subsection{Introduction}

When ionizing radiations such as $\mathrm{x}$ rays, gamma rays, and electrons interact with tissue, the inflicted damage (per unit dose) generally increases with decreasing particle energy. ${ }^{73,74}$ This is due to the increase in ionization density and more interactions with DNA along the particle's track. There are two main modes of treating cancer using ionizing radiation: external beam radiotherapy (EBRT) and brachytherapy. Typically, photon energies greater than $1 \mathrm{MeV}$ are used in EBRT in order to achieve the necessary tissue penetrating power required to reach tumors situated deep within the body. Since the photon energies used in EBRT are relatively high, the variation of relative biological effectiveness (RBE) is usually minimal. It has been demonstrated that 
maximum RBE can change by up to $16 \%$ when complex beam cases are used due to the presence of increased scattered (low energy) radiation. ${ }^{22}$

In brachytherapy, the radioactive source(s) are placed inside the patient to minimize entrance and exit doses relative to EBRT. Brachytherapy may be more susceptible to RBE variations given that the radioactive sources used are lower in energy. In low-dose rate (LDR) treatments, the radioisotope ${ }^{125} \mathrm{I}$ is most commonly used, whereas ${ }^{192} \mathrm{Ir}$ is most common in high-dose rate (HDR) treatments. ${ }^{75,76}$ There are several other radioisotopes that have also been explored in brachytherapy; these include ${ }^{169} \mathrm{Yb},{ }^{75} \mathrm{Se}$, ${ }^{153} \mathrm{Gd}$, and ${ }^{103} \mathrm{Pd}$. These sources have different energy spectra and therefore have different mean energies (Table 4.1).

Table 4.1: Mean energies of various radioactive sources commonly used in brachytherapy. ${ }^{77}$

\begin{tabular}{|cc|}
\hline Source & $\begin{array}{c}\text { Mean Y Energy } \\
\text { (keV) }\end{array}$ \\
\hline${ }^{103} \mathrm{Pd}$ & 21 \\
\hline${ }^{125} \mathrm{I}$ & 30 \\
\hline${ }^{153} \mathrm{Gd}$ & 60 \\
\hline${ }^{169} \mathrm{Yb}$ & 93 \\
\hline${ }^{75} \mathrm{Se}$ & 210 \\
\hline${ }^{192} \mathrm{Ir}$ & 380 \\
\hline${ }^{137} \mathrm{Cs}$ & 662 \\
\hline${ }^{60} \mathrm{Co}$ & 1,250 \\
\hline
\end{tabular}


Since it is known that lower energy particles are more damaging, the question of whether RBE changes and if so by how much has been posed many times before. ${ }^{2477-}$ ${ }^{80}$ In this section, the previous works on RBE determination using ${ }^{125} \mathrm{I},{ }^{192} \mathrm{Ir}$, and ${ }^{169} \mathrm{Yb}$ will be examined. The review of each source will be subdivided into two main types of RBE evaluation methods: 1) animal tumour models, and 2) based on experimental microdosimetry.

\section{$\underline{\text { lodine-125 }}$}

RBE of ${ }^{125}$ I, a commonly used LDR source, has been examined many times using animal tumour models. Bernstein et al. ${ }^{81}$ found its RBE to be 1.0 relative to ${ }^{137} \mathrm{Cs}$. RIF-1 murine flank tumors were irradiated under high dose and high dose rate conditions and survival was then examined in order to obtain this result. This was followed by Freeman et al. ${ }^{82}$ using Chinese hamster ovary cells to determine RBE for different phases of the cell cycle. It was found that at high dose rates RBE of ${ }^{125}$ I was 1.2 while it increased up to 2.0 at low dose rates; the reference radiation used was ${ }^{137} \mathrm{Cs}$. Da Silva et al. ${ }^{83}$ experimentally determined that RBE of ${ }^{125}$ I was 1.0 relative to ${ }^{192}$ Ir. In 1985 , Kwan et al. ${ }^{84}$ determined that the RBE of ${ }^{125}$ I was 1.8 and 1.6 when $4 \mathrm{MeV}$ x-rays and ${ }^{137} \mathrm{Cs}$, respectively, were used as reference radiation. This study examined the survival of blood lymphocytes when exposed to various radiation sources. Hering et al. ${ }^{85}$ examined how RBE changed as the oxygen enhancement changed in B16 melanoma cells. The reference radiation used was ${ }^{192}$ Ir and RBE was found to range from 1.8 (aerated) to 2.4 (hypoxic).

There are also several works which have taken a microdosimetric approach. Wuu et al. ${ }^{86}$ found RBE of various brachytherapy sources at low doses and dose rates using a 
grid-defined wall-less proportional counter to measure the lineal energy transfer. It was found that the RBE of ${ }^{125} \mathrm{I}$ relative to ${ }^{60} \mathrm{Co}$ was 2.1 . Interestingly, at the same time, Ling et al. ${ }^{87}$ determined an RBE of 1.4 for ${ }^{125} \mathrm{I}$ relative to ${ }^{60} \mathrm{Co}$ also at low dose rates $(0.07$ Gy/h). Famulari et al. ${ }^{77}$ used a microdosimetric approach to determine the RBE of various brachytherapy sources. Using the Geant4-DNA package, they obtained an RBE of 1.17 for ${ }^{125}$ ( $(1 \mathrm{MeV}$ photons used as reference). It is difficult to compare these results given the various different conditions under which these studies were conducted. The reference radiation and cell type varied and RBE values ranging from 1.0 to 2.4 were reported.

\section{Iridium-192}

RBE values for ${ }^{192} \mathrm{Ir}$ in the literature range from 1.3 to 2.0 . As described by Wuu et al., ${ }^{86}{ }^{192}$ Ir radioactive source's energy spectrum contains a range of photons, $62.7 \%$ of which are in $200 \mathrm{keV}$ energy range along with the $21.3 \%$ in the $460 \mathrm{keV}$ range. Due to this, $99 \%$ of the secondary electrons produced in water from ${ }^{192}$ Ir interaction are created by the Compton effect. Since the radiation emitted is higher in energy relative to other common brachytherapy sources $\left({ }^{125} \mathrm{I},{ }^{169} \mathrm{Yb}\right),{ }^{192} \mathrm{Ir}$ is commonly used as the reference radiation for RBE calculations of lower energy sources, electronic brachytherapy, and intraoperative radiotherapy. ${ }^{88-92}$ Also, due to its higher energy, the RBE for ${ }^{192}$ Ir (relative to ${ }^{60} \mathrm{Co}$ ) is close to unity.

Experimentally, ${ }^{192} \mathrm{Ir}$ is typically used as the reference radiation for lower energy brachytherapy sources such as ${ }^{125} \mathrm{I}$ or $\mathrm{EBT}^{93,94}$ (electronic brachytherapy); thus, animal model RBE results are limited. A microdosimetric approach to determine RBE was taken by Wuu et al. ${ }^{86}$ By calculating the lineal energy transfer using proportional 
counters, they were able to determine ${ }^{192}$ Ir RBE to be 1.3 with ${ }^{60} \mathrm{Co}$ as the reference radiation. Zellmer et al. ${ }^{95}$ determined that ${ }^{192} \mathrm{Ir}$ RBE at low dose rates was 1.29 (relative to ${ }^{60} \mathrm{Co}$ ); this was obtained by measuring the single event microdosimetric spectra using a Rossi proportional counter. This group then used a similar method to determine RBE at low doses; ${ }^{192}$ Ir RBE (relative to ${ }^{60} \mathrm{Co}$ ) was found to increase to $1.5 .{ }^{96}$ As described above when discussing ${ }^{125} \mathrm{I}$, Famulari et al. ${ }^{77}$ used a microdosimetric approach and determined an RBE of ${ }^{192} \mathrm{Ir}$ (relative to $1 \mathrm{MeV}$ photons) to be equal to 1.028 .

\section{$\underline{\text { Ytterbium-169 }}$}

Radioactive ${ }^{169} \mathrm{Yb}$ sources were developed for use in brachytherapy in the 1980 s; however, its popularity in the clinic has not yet reached predicted levels and it is not available today. Advantages for the use of ${ }^{169} \mathrm{Yb}$ included its ideal half-life (32 days) for permanent implants, low toxicity, and having a high specific activity. ${ }^{84,95,97}$ Given that the average energy of emitted photons is only $93 \mathrm{keV}$, potential radiobiological consequences may be a concern. Using the animal model method of determining RBE, Plume et al. ${ }^{98}$ determined that RBE ranged from 1.1 to $1.3\left({ }^{169} \mathrm{Yb}\right.$ relative to $\left.{ }^{60} \mathrm{Co}\right)$ depending on the dose rate. In their study, Chinese hamster ovary cells (exponential growth phase) were exposed to both radiation types $\left({ }^{169} \mathrm{Yb}\right.$ and $\left.{ }^{60} \mathrm{Co}\right)$ at various dose rates. Using microdosimetric techniques, Zellmer et al. ${ }^{99}$ calculated mean lineal energy transfers and used them to determine the RBE for ${ }^{169} \mathrm{Yb}$ to be 1.60 using ${ }^{60} \mathrm{Co}$ as the reference radiation. In the same study discussed earlier in the introduction $\left({ }^{125} \mathrm{I}\right.$ and ${ }^{192} \mathrm{Ir}$ literature reviews), Famulari et al. ${ }^{77}$ found an RBE of 1.05 for ${ }^{169} \mathrm{Yb}$ relative to $1 \mathrm{MeV}$ photons. 
Table 4.2: The summary of previously determined RBE values for ${ }^{125} \mathrm{I},{ }^{192} \mathrm{Ir}$, and ${ }^{169} \mathrm{Yb}$ is shown below.

\begin{tabular}{|c|c|c|c|c|}
\hline \multirow{2}{*}{$\begin{array}{c}\text { Source } \\
{ }^{125} \mid\end{array}$} & RBE & Ref. Radiation & Ref. & RBE Technique \\
\hline & 1.0 & ${ }^{137} \mathrm{Cs}$ & & Animal Model \\
\hline & $1.2-2.0$ & ${ }^{137} \mathrm{Cs}$ & 82 & Animal Model \\
\hline & 1.0 & ${ }^{192} \mathrm{Ir}$ & 83 & Animal Model \\
\hline & 1.8 & $4 \mathrm{MeV}$ x-rays & 84 & Animal Model \\
\hline & 1.6 & ${ }^{137} \mathrm{Cs}$ & 84 & Animal Model \\
\hline & $1.8-2.4$ & ${ }^{192} \mathrm{Ir}$ & 85 & Animal Model \\
\hline & 2.1 & ${ }^{60} \mathrm{Co}$ & 86 & Microdosimetry \\
\hline & 1.4 & ${ }^{60} \mathrm{Co}$ & 87 & Microdosimetry \\
\hline & 1.17 & $1 \mathrm{MeV}$ photons & 77 & Microdosimetry \\
\hline${ }^{192} \mathrm{Ir}$ & 1.3 & ${ }^{60} \mathrm{Co}$ & 86 & Microdosimetry \\
\hline & 1.29 & ${ }^{60} \mathrm{Co}$ & 95 & Animal Model \\
\hline & 1.5 & ${ }^{60} \mathrm{Co}$ & 96 & Microdosimetry \\
\hline & 1.028 & $1 \mathrm{MeV}$ photons & 77 & Microdosimetry \\
\hline${ }^{169} \mathrm{Yb}$ & 1.60 & ${ }^{60} \mathrm{Co}$ & 95 & Microdosimetry \\
\hline & $1.1-1.3$ & ${ }^{60} \mathrm{Co}$ & 98 & Animal Model \\
\hline & 1.05 & $1 \mathrm{MeV}$ photons & 77 & Microdosimetry \\
\hline
\end{tabular}

As seen in Table 4.2, there is a disagreement in the literature regarding what the RBE values of ${ }^{125} \mathrm{I},{ }^{192} \mathrm{Ir}$, and ${ }^{169} \mathrm{Yb}$ really are. Additionally, previous studies only examined RBE change at the source position. The spatial variation in the region surrounding the radioactive source has not previously been looked at.

In brachytherapy, treatment plans are created by choosing source dwell positions and dwell times that result in optimal radiation absorbed dose to the tumour while minimizing the dose to healthy tissues nearby. The potential impact of RBE variation is 
not considered in current brachytherapy treatment planning; this may be potentially causing the effective dose in the low-dose regions to be greater than previously thought. Several studies have commented on the need for RBE-based treatment planning in brachytherapy. ${ }^{20,24,26,77,100-103}$

The purpose of this study was to examine the spatial variation of RBE surrounding a radioactive source. First, this was examined for three single source cases: ${ }^{125} \mathrm{I},{ }^{192} \mathrm{Ir}$, and ${ }^{169} \mathrm{Yb}$. Following this, RBE variation in an HDR prostate treatment plan was examined. In this work, a microdosimetry approach chosen to be the more appropriate approach given that the goal is to examine RBE variation in the low-dose regions.

\subsection{Materials and Methods}

In this study, the change in maximum relative biological effectiveness (RBE) surrounding three radioactive sources $\left({ }^{125} \mathrm{I},{ }^{192} \mathrm{Ir}\right.$, and $\left.{ }^{169} \mathrm{Yb}\right)$ was examined. Generally, RBE is calculated by normalizing the absorbed dose of the test radiation required to reach a given biological endpoint (for example, $10 \%$ cell survival) to the absorbed dose of some reference radiation required to reach the same endpoint. In this study, the test radiation was the electron energy fluence spectrum present at a given distance away from the source while the reference radiation used was the electron energy fluence at the source's position. Thus, an RBE distribution around the source relative to the RBE at the position of the source center was obtained. In order to validate the results, RBE of

${ }^{125}$ I was determined relative to ${ }^{192}$ Ir for comparison and validation with previous works. Once this was done, the maximum RBE distribution for each of the three sources 
examined was obtained. In the second part of this study, the RBE distribution of ${ }^{192} \mathrm{Ir}$ was used to re-evaluate an HDR prostate treatment plan in terms of RBE in addition to absorbed dose.

\subsubsection{Radioactive Sources}

The radioactive sources used in this work were ${ }^{125} \mathrm{I},{ }^{192} \mathrm{Ir}$, and ${ }^{169} \mathrm{Yb}$. The sources were selected to span the typical range of energies encountered in brachytherapy. In the Monte Carlo simulation, the energy spectra (Figure 4.1) for the radioactive sources were obtained from the CLRP TG-43 Parameter Database for Brachytherapy. ${ }^{104}{ }^{125}$ | source used in this work was the 'Nucletron SelectSeed 130.002 (`'. The source's geometry and dimensions were obtained from Karaiskos et al. ${ }^{105}$ and were replicated in the simulation. Since the energy spectrum obtained from the CLRP TG-43 Database took beam attenuation due to source casing into account and was representative of the photon energy spectrum just outside the casing, the $3.00 \mu \mathrm{m}$ silver halide wall surrounding the radioactive ${ }^{125} \mathrm{I}$ was not modeled in the simulation. ${ }^{192}$ Ir radioactive source used was a model of the 'Isodose Control Flexisource HDR @'. The geometry and dimensions of this ${ }^{192}$ Ir source were created in the simulation in accordance with Granero et al. ${ }^{76}$ The casing surrounding the source was not modelled. The ${ }^{169} \mathrm{Yb}$ source used in this work was the 'Implant Sciences Corporation 4140 HDR @'. Source geometry and dimensions were obtained from Medich et al. ${ }^{106}$ 


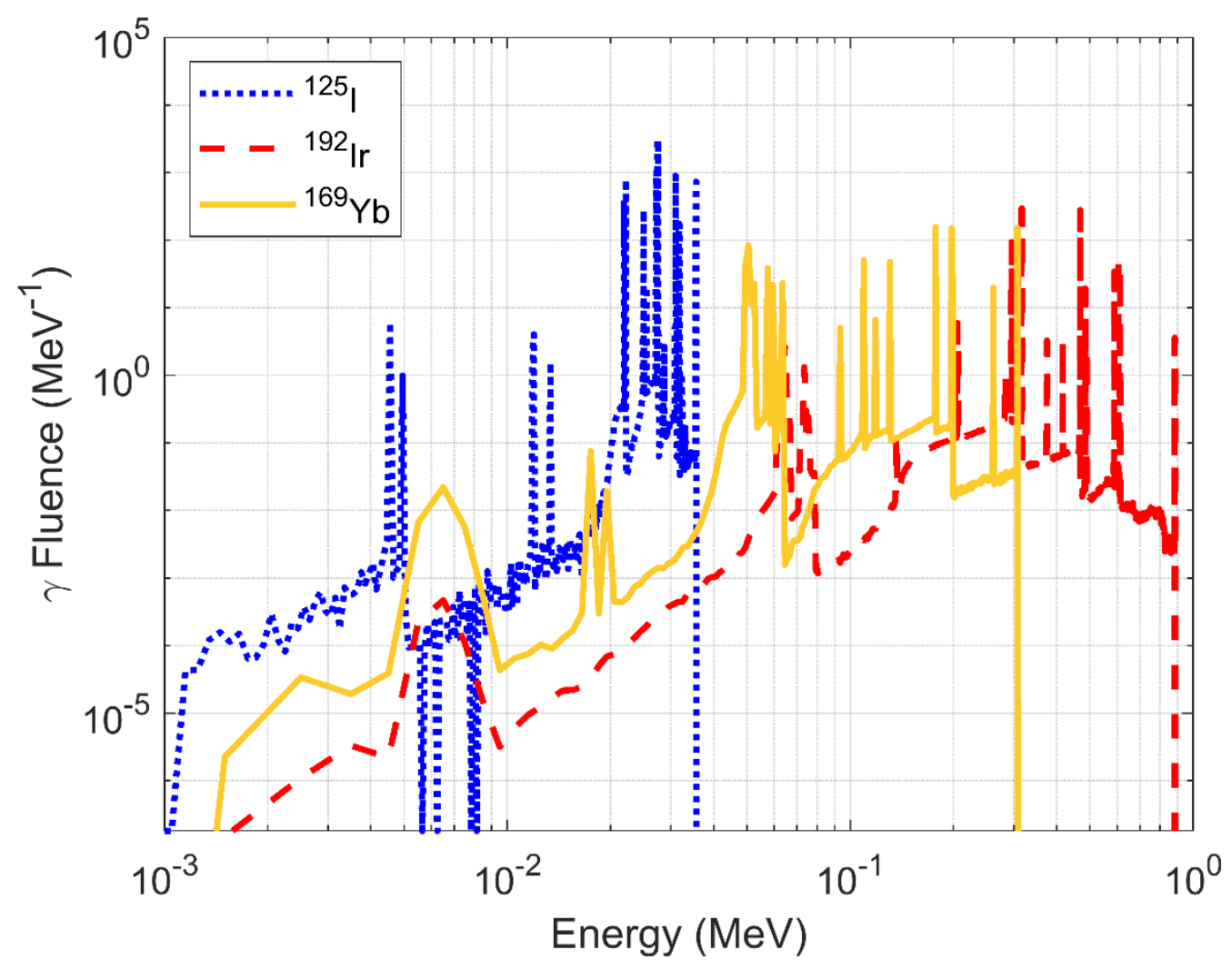

Figure 4.1: Energy weighted photon spectra for ${ }^{125} \mathrm{I}$ (blue; dotted), ${ }^{192} \mathrm{Ir}$ (red; dashed), and ${ }^{169} \mathrm{Yb}$ (yellow; solid) from Taylor et al. ${ }^{107}$ The radioactive source geometry surrounded by water was recreated in Geant4; the data shown above was used as the photon emission spectra.

\subsubsection{Electron Energy Fluence, $n(E)$}

In this study, the radioactive sources were modelled using Geant4.10.3 Monte Carlo simulations and their resulting electron energy spectra in water were determined. 108 One source was placed at the center of a $16 \times 16 \times 16 \mathrm{~cm}^{3}$ water tank, and the 3 dimensional electron energy fluence was scored at all positions using $2 \times 2 \times 2 \mathrm{~mm}^{3}$ 
voxels. In Geant4, the 'EmStandardPhysics_option4' package was used for the simulation. Photon and electron range cuts of $100 \mathrm{~nm}$ were used. For all cases, the Monte Carlo simulations were run for $2.2 \times 10^{9}$ initial histories, yielding an absorbed dose uncertainty better than $0.1 \%$ at $1 \mathrm{~cm}$ away from the source in water. The electron energy spectra produced by the brachytherapy sources correspond to the $\mathrm{nl}$ component in Equation 4.2. As per AAPM TG268 report, the simulation parameters and features used in this study are described in Table 4.3. ${ }^{109}$ The photon and electron fluence spectra were collected and shown at the source position and the periphery in order to explain resulting $\mathrm{RBE}_{\mathrm{M}}$ differences.

Table 4.3: Summary of Monte Carlo parameters used to create the simulation for obtaining RBE values of ${ }^{125} \mathrm{I},{ }^{192} \mathrm{Ir}$, and ${ }^{169} \mathrm{Yb}$. This table is based on AAPM TG268 Report and 'TG268 Item \#' refers to the required Monte Carlo item specified in Sechopolous et al. ${ }^{109}$

\begin{tabular}{|lllc|}
\hline $\begin{array}{l}\text { TG268 } \\
\text { Item \# }\end{array}$ & Item Name & Description & Ref. \\
\hline 2,3 & $\begin{array}{l}\text { Code, } \\
\text { version/rel. date }\end{array}$ & $\begin{array}{l}\text { Geant4 Version 10.3 } \\
\text { Released - Dec. 9 2016 }\end{array}$ & 108 \\
\hline 4,17 & Validation & Refer to Carrier et al., 2004 & 47 \\
\hline 5 & Timing & $\begin{array}{l}\text { Simulation time: } 6 \mathrm{~h} \\
\text { CPU: Intel Xenon; 20 Cores }\end{array}$ & 107 \\
\hline 8 & $\begin{array}{l}\text { Source } \\
\text { description }\end{array}$ & $\begin{array}{l}\text { (25/, }{ }^{192} \text { Ir, and }{ }^{169} \text { Yb x ray spectra after casing } \\
\text { obtained from CLRP brachytherapy database }\end{array}$ & 107 \\
\hline 9 & Cross-sections & $\begin{array}{l}\text { Physics Package: } \\
\text { EMStandardPhysics_Option4 }\end{array}$ & 110 \\
\hline
\end{tabular}




\begin{tabular}{|c|c|c|c|}
\hline & & $\begin{array}{l}\text { - PE: G4LivermorePhotoElectricModel } \\
\text { - Compton: G4LivermoreComptonModel (0- } \\
\text { 2MeV), G4KleinNishinaModel (2 MeV) } \\
\text { - GK: G4PenelopeGammaConversionModel } \\
\text { - Rayleigh: G4RayleighScattering }\end{array}$ & \\
\hline 10 & $\begin{array}{l}\text { Transport } \\
\text { parameters }\end{array}$ & $\begin{array}{l}\text { - Physics Package: } \\
\text { EMStandardPhysics_Option4 } \\
\text { - Multiple Scattering: G4UrbanMscModel95 } \\
\text { - Ionization: G4PenelopelonisationModel } \\
\text { - Brem: G4SeltzerBergerModel } \\
\text { - G4Cutoff: } 100 \mathrm{~nm} \text { e-, e+, y }\end{array}$ & 110 \\
\hline 11 & $\begin{array}{l}\text { VRT and/or } \\
\text { AEIT }\end{array}$ & $\mathrm{N} / \mathrm{a}$ & \\
\hline 12 & $\begin{array}{l}\text { Scored } \\
\text { quantities }\end{array}$ & $\begin{array}{l}\text { Electron energy fluence using command based } \\
\text { Geant4 scoring. Logarithmically increasing } \\
\text { energy bins ranging from } 920 \mathrm{eV} \text { to } 1.6 \mathrm{MeV} \\
\text { used. }\end{array}$ & \\
\hline 13,18 & $\begin{array}{l}\text { \# of histories/ } \\
\text { statistical } \\
\text { uncertainty }\end{array}$ & $\begin{array}{l}\text { - } 2.2 \times 10^{9} \text { histories per simulation } \\
\text { - Absorbed dose uncertainty }<0.1 \% 1 \mathrm{~cm} \text { away } \\
\text { from source }\end{array}$ & \\
\hline 14 & $\begin{array}{l}\text { Statistical } \\
\text { methods }\end{array}$ & $\begin{array}{l}\text { - Absorbed dose uncertainty calculated as root } \\
\text { mean square of energy deposited normalized } \\
\text { to mass } \\
\text { - Starting seed \# changed and } \mathrm{RBE}_{\mathrm{M}} \text { calculated } \\
\text { multiple times }(n=5) \text {; standard error on } \mathrm{RBE}_{\mathrm{M}} \\
\text { was obtained }\end{array}$ & \\
\hline 15,16 & Post-processing & $\begin{array}{l}\text { Raw electron energy fluence converted to } \mathrm{RBE}_{\mathrm{M}} \\
\text { using the method described in Section } 3.3\end{array}$ & 22 \\
\hline
\end{tabular}




\subsubsection{Calculating Relative Biological Effectiveness (RBE)}

Since RBE variation under low-dose conditions was examined, the maximum RBE, $R_{B E}$ was the quantity of interest. In this study, the approach used by Nusrat et al. $^{22}$ was used to generate $\mathrm{RBE}_{\mathrm{M}}$. According to the theory of dual radiation action, the monoenergetic electron $\alpha / \beta$ can be obtained through:

$$
\frac{\alpha}{\beta}(E)=\int_{0}^{\infty} t(x, E) \gamma(x) d x
$$

In this equation, derived from Kellerer and Rossi's theory of dual radiation action, the $\alpha / \beta$ ratio for monoenergetic electrons is obtained. ${ }^{68}$ As per the linear quadratic model, the $\alpha / \beta$ ratio is defined as the dose at which cells killed via double and single stranded DNA breakages are equivalent. The proximity function, $t(x, E)$, represents the probability that an electron of energy, $E$, undergoes subsequent energy depositions at distance, $x$. Proximity function data for electrons in water of energies ranging from 100 $\mathrm{eV}$ to $10 \mathrm{MeV}$ were obtained from Chen and Kellerer. ${ }^{69}$ The gamma function, $\gamma(x)$, represents the probability that adjacent energy depositions at distance, $x$, result in a lesion and must be obtained through measurement for a given cell line. ${ }^{67}$ This component is only dependent upon the cell line used; in this work, the V79 Chinese hamster cell was the biological medium. ${ }^{67}$

Equation 4.1 represents a monoenergetic case. If a polyenergetic energy spectrum of electrons is examined; the energy weighted $\alpha / \beta$ ratio $(\overline{\alpha / \beta})$ can be calculated using

$$
\overline{\alpha / \beta}=\frac{\int_{0}^{\infty} \frac{\alpha}{\beta}(E) n(E) E d E}{\int_{0}^{\infty} n(E) E d E},
$$


where $n l$ is the energy fluence spectrum of electrons present locally, $E$ is the energy of the electrons, and $\alpha / \beta /$ is the monoenergetic $\alpha / \beta$ ratio as calculated in Equation 4.1. As shown by Dale and Jones, at the low dose limit, the ratio of $\overline{\alpha / \beta}$ values becomes the maximum RBE, $\mathrm{RBE}_{\mathrm{M}}{ }^{66}$ In this work, spatial variation in $\mathrm{RBE}_{\mathrm{M}}$ was calculated by normalizing $\overline{\alpha / \beta}(x)$ at a point in space to $\overline{\alpha / \beta}(x=0 \mathrm{~cm})$ at the source location. In order to validate our results with published literature, the $\mathrm{RBE}_{\mathrm{M}}$ of ${ }^{125} \mathrm{I}$ using ${ }^{192} \mathrm{Ir}$ as the reference radiation was also calculated.

\subsection{4. $\mathrm{RBE}_{\mathrm{M}}$ Distribution of a Typical Prostate Brachytherapy Treatment}

In order to examine potential variability on a clinical treatment, the $\mathrm{RBE}_{\mathrm{M}}$ distribution for a typical prostate plan was calculated. Oncentra® Version 3.1 (Nucletron V.B., Veenendaal, The Netherlands) was used to create an HDR treatment plan for a typical prostate case that used 16 catheter needles. Source positions and dwell times were exported to MATLAB. At each source position, the 3-dimensional electron fluence described in Section 4.3.2 was placed; the electrons present in overlapping voxels were summated. Dwell time weighting was applied by multiplying the entire summated plan fluence with a 3-dimensional dwell time matrix. Once a realistic 3-dimensional fluence distribution was obtained, one slice was chosen for evaluation purposes. 


\subsection{Results}

Results of this study are presented in two main parts: 1) single source $R_{B E}$ distribution, and 2) $\mathrm{RBE}_{\mathrm{M}}$ evaluation for a typical HDR prostate plan. The $\mathrm{RBE}_{\mathrm{M}}$ of ${ }^{125}$ I relative to ${ }^{192}$ Ir was determined to be $1.558(+/-0.002)$ (Figure 4.2). The single source $\mathrm{RBE}_{\mathrm{M}}$ distribution is shown in Figure 4.3 below. $\mathrm{RBE}_{\mathrm{M}}$ of ${ }^{125} \mathrm{I},{ }^{192} \mathrm{Ir}$, and ${ }^{169} \mathrm{Yb}$ at $8 \mathrm{~cm}$ away from the source was $0.994(+/-0.002), 1.030(+/-0.003)$, and $1.066(+/-0.008)$, respectively. For both figures 4.2 and 4.3 , the $\mathrm{RBE}_{M}$ is shown perpendicular to the longitudinal axis of each brachytherapy source. Electron and photon energy fluence spectra at the source position $(x=0 \mathrm{~cm})$ and the periphery $(x=8 \mathrm{~cm})$ are shown in Figure 4.4. In order to compare sources, the axes in Figure 4.4 were kept constant; thus, the larger low energy component at the source position of ${ }^{125} \mathrm{I}$ is not visible. The ${ }^{125}$ I mean electron energy for the source position was $28.5 \mathrm{keV}$; at the periphery $(8 \mathrm{~cm}$ away) this increased to $28.7 \mathrm{keV}$. In Figure 4.5, the treatment plan re-evaluated in terms of $R_{B E}$ is shown. The electron fluence (Fig. 4.5A) and corresponding $R_{B} E_{M}$ distribution (Fig. 4.5B) for a single slice bisecting the PTV, respectively, are shown. It was observed that $\mathrm{RBE}_{\mathrm{M}}$ increased by up to $3.6 \%$ (relative to ${ }^{192}$ Ir source) in the peripheral regions of the scoring volume. 


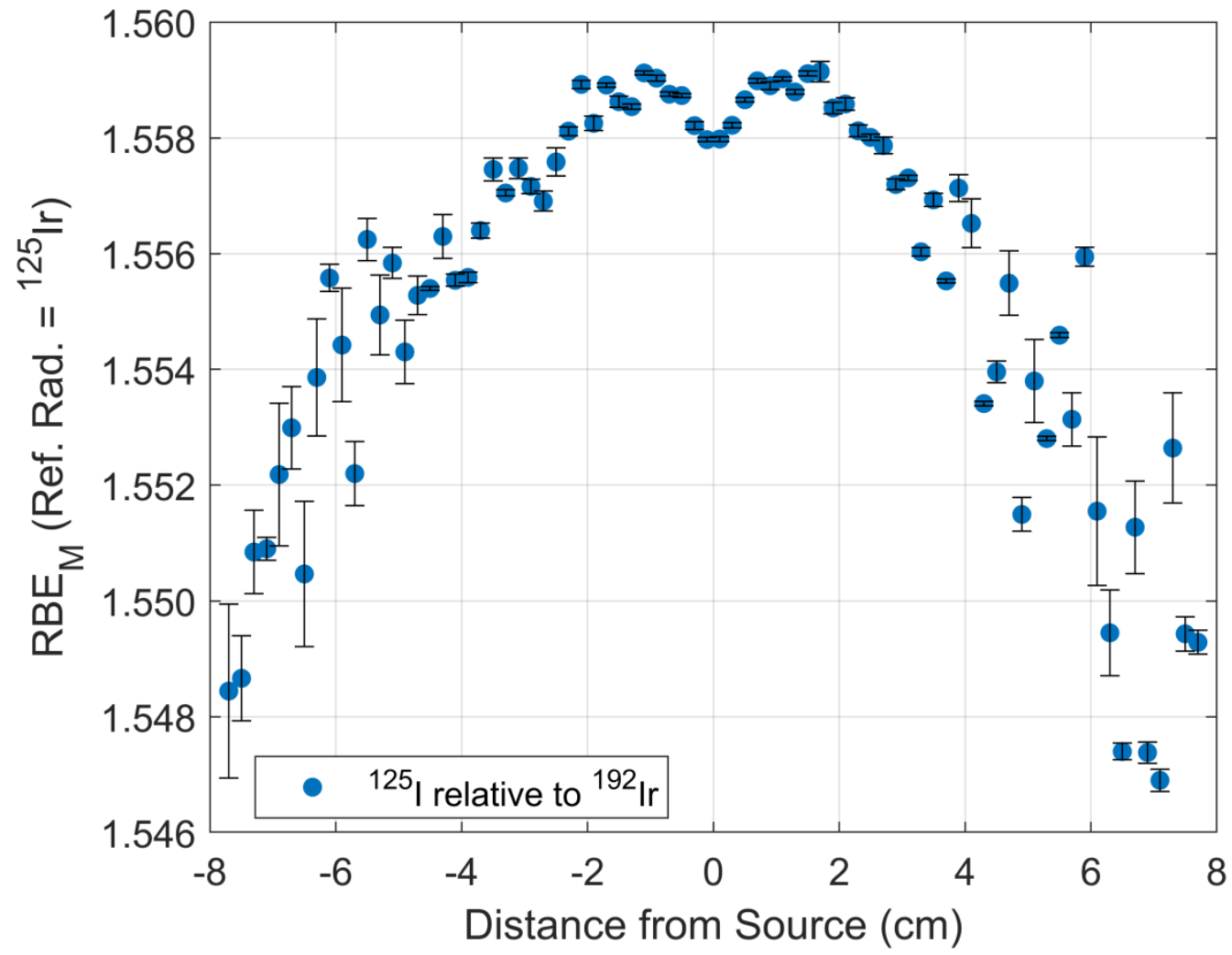

Figure 4.2: $\mathrm{RBE}_{\mathrm{M}}$ shown for ${ }^{125} \mathrm{I}$ when ${ }^{192} \mathrm{Ir}($ at $x=0 \mathrm{~cm}$ ) was used as the reference radiation. Error bars are representative of the standard error of the mean $R_{B} E_{M}$ calculated using multiple $(n=5)$ simulations with different starting seed numbers. 


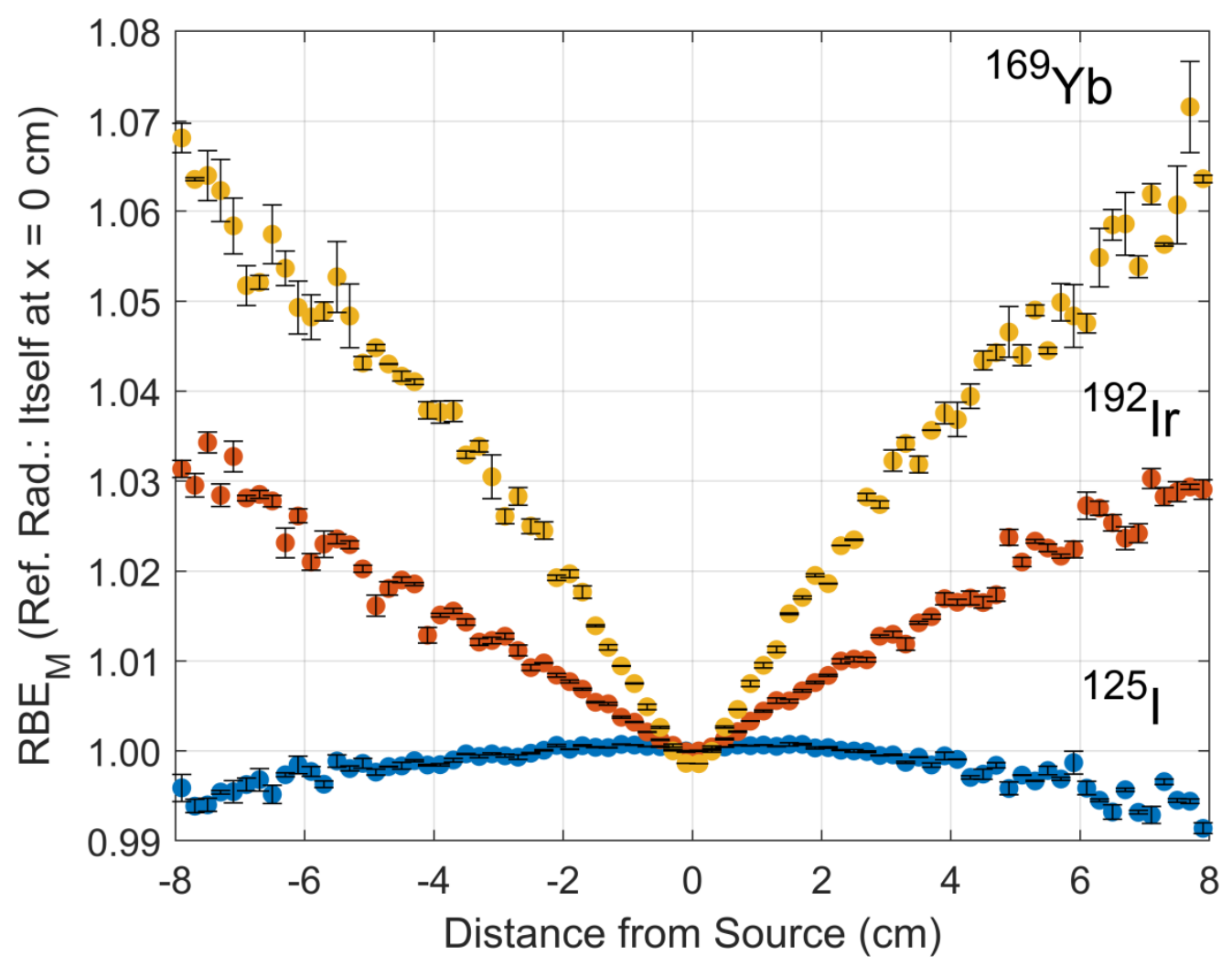

Figure 4.3: $\mathrm{RBE}_{\mathrm{M}}$ as a function of distance from the source $(x=0 \mathrm{~cm})$. Data for three sources are shown above, ${ }^{125} \mathrm{I}$ (blue), ${ }^{192} \mathrm{Ir}$ (red), and ${ }^{169} \mathrm{Yb}$ (yellow). For each source, the electron fluence present in the voxel at position, $x$, was used as the test radiation, and the electron fluence at the position $x=0 \mathrm{~cm}$ (source) was used as the reference radiation. Error bars are representative of the standard error of the mean $\mathrm{RBE}_{\mathrm{M}}$ at each position calculated using multiple $(n=5)$ simulations, each with different initial seed numbers. 

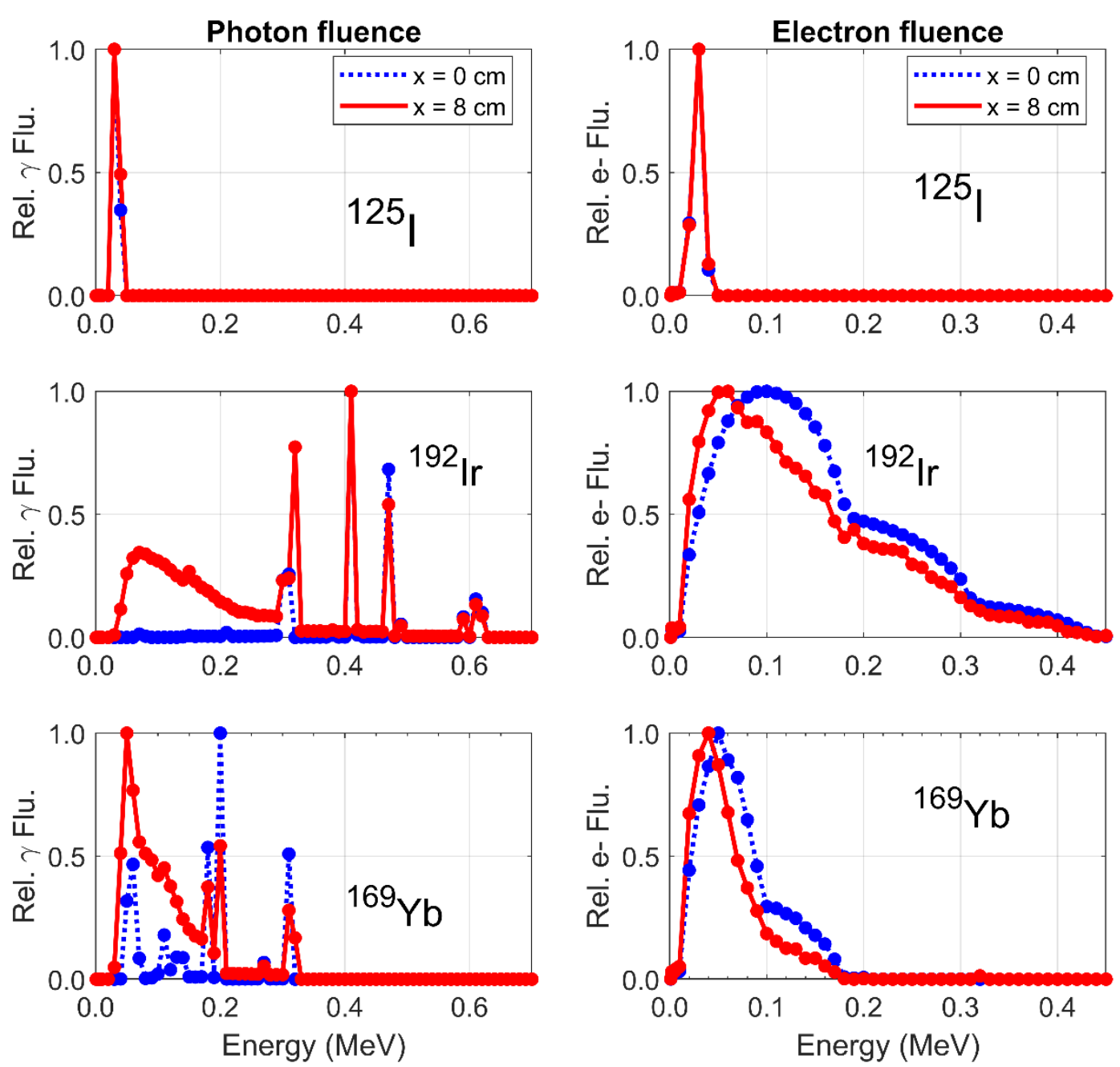

Figure 4.4: Photon and electron energy fluence spectra at the source position (dotted line) and $8 \mathrm{~cm}$ (solid line) in water. The electron fluence spectra ${ }^{192} \mathrm{Ir}$ and ${ }^{169} \mathrm{Yb}$ are different when comparing the source position and the periphery (resulting in the increased $R B E_{M}$ ). Axes were kept consistent in order to compare spectra between the three sources. ${ }^{125} \mathrm{I}$ mean electron energy for the source position was $28.5 \mathrm{keV}$; at the periphery ( $8 \mathrm{~cm}$ away) this increased to $28.7 \mathrm{keV}$. 

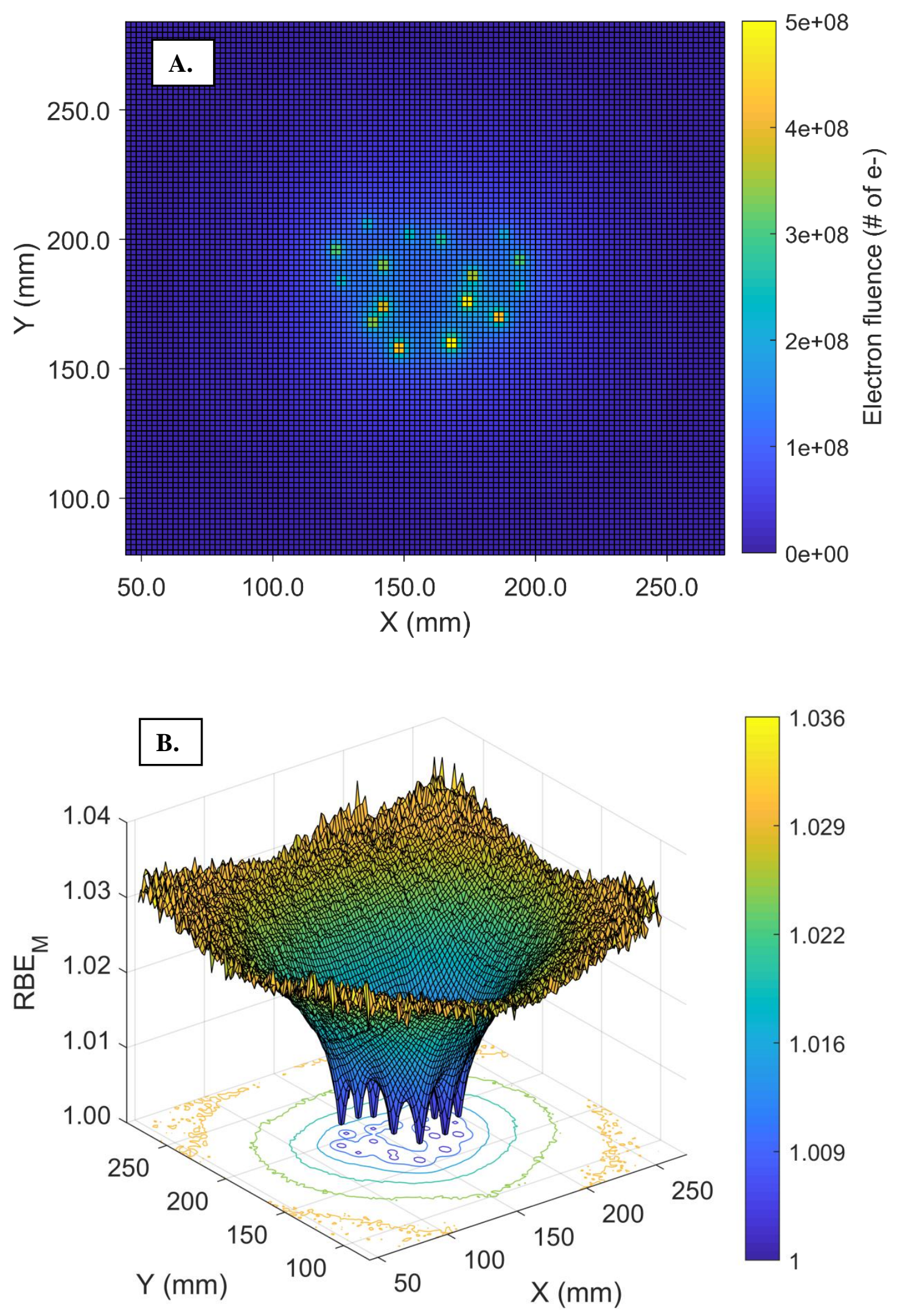
Figure 4.5: (A) The resulting ${ }^{192}$ Ir HDR prostate treatment plan's electron fluence in a slice bisecting the PTV. Bright spots indicate a high number of electrons present (maximum of $5 \times 10^{8}$ ) while dark spots indicate few or no electrons. (B) Single slice $\mathrm{RBE}_{M}$ distribution calculated using the electron fluence shown in Figure $5 \mathrm{~A}$. $\mathrm{RBE}_{\mathrm{M}}$ values of 1.00 represent no change relative to the source position; $R_{B E}$ of 1.036 represents a $3.6 \%$ increase. A projection of the 2-dimsional $\mathrm{RBE}_{\mathrm{M}}$ distribution is shown in the $\mathrm{X}-\mathrm{Y}$ plane.

\subsection{Discussion}

As discussed in the introduction, there is significant disagreement among previous studies regarding the RBE of the three radioactive sources analyzed. Table 4.1 summarizes the results found in literature. For ${ }^{125} \mathrm{I}$, values range from 1.0 to 2.4 depending on the dose, dose rate, and choice of reference radiation. In our study, RBE under low dose conditions was examined in order to evaluate potential impact on organs at risk (OAR) in brachytherapy. In order to validate our work against previously published literature, the $\mathrm{RBE}_{\mathrm{M}}$ of ${ }^{125} \mathrm{I}$ was determined with ${ }^{192} \mathrm{Ir}$ as the reference radiation (Figure 4.3).$^{84,85,111-113}$ Hering ${ }^{113}$ irradiated Vicia faba with both ${ }^{125} \mathrm{I}$ and ${ }^{192} \mathrm{Ir}$ and determined an RBE of 1.5 , consistent with the $1.558 \mathrm{RBE}_{M}$ determined in our study. Kwan et al. ${ }^{84}$ determined ${ }^{125} \mathrm{I} \mathrm{RBE}$ of 1.6 and 1.8 when ${ }^{137} \mathrm{Cs}$ and $4 \mathrm{MeV} \mathrm{x}$ rays were used as the reference radiation, respectively. In that study, RBE was determined experimentally; human lymphocytes were used as the biological medium and the results were reported at 100 cGy absorbed dose (low dose conditions). Given that the ${ }^{137} \mathrm{Cs} \mathrm{X}$ - 
ray energy spectrum extends to higher energies than the ${ }^{192} \mathrm{Ir}$ spectrum, our reported $\mathrm{RBE}_{\mathrm{M}}$ of 1.558 seems to be consistent with their results. In another study, Lehnert et al. ${ }^{111}$ determined an RBE of $1.4-1.5$ for ${ }^{125}$ I relative to ${ }^{60} \mathrm{Co}$. In their work, RIF-1 mouse tumors were irradiated by temporarily implanting a ${ }^{125} \mathrm{I}$ source or using ${ }^{60} \mathrm{Co}$ gamma rays. Accounting for the difference between $R B E$ and $R B E_{M}$ (recall that $R B E_{M}$ is the maximum RBE observed at the low dose limit), the results determined in our study using Monte Carlo are consistent with their experimentally determined RBE.

Currently, the prevalence of RBE-weighting in the treatment planning process is not ubiquitous despite numerous suggestions in literature. ${ }^{24,77,78,88,89,101,103,114-123}$ Contrary to previous works, this study sought to determine how RBE changed as a function of distance from the source and the resulting impact on a treatment plan. Figure 4.3 presents the results for ${ }^{125} \mathrm{I},{ }^{192} \mathrm{Ir}$, and ${ }^{169} \mathrm{Yb}$. First, $\mathrm{RBE}_{\mathrm{M}}$ of ${ }^{125} \mathrm{I}$ was found to decrease slightly as distance from the source increased. At a distance of $8 \mathrm{~cm}$ away from the source position, the $\mathrm{RBE}_{\mathrm{M}}$ decreased to 0.994 . Essentially, the electrons present in the $2 \mathrm{~mm}^{3}$ local volume $8 \mathrm{~cm}$ away were $0.6 \%$ less biologically effective at cell killing. This slight decrease was observed in Figure 4.2 as well $\left({ }^{125} \mathrm{I}\right.$ relative to $\left.{ }^{192} \mathrm{Ir}\right)$. This decrease, while minute, is an interesting observation. It is most likely caused by attenuation of a small number of very low energy gamma rays close to the source. This difference can also be seen when comparing the mean electron energies at the two positions (27.5 $\mathrm{keV}$ at source, $27.8 \mathrm{keV}$ at the periphery).

In contradiction to ${ }^{125} \mathrm{I}$, the $\mathrm{RBE}_{\mathrm{M}}$ of ${ }^{192} \mathrm{Ir}$ and ${ }^{169} \mathrm{Yb}$ was found to increase with distance away from the source by approximately $3 \%$ and $7 \%$, respectively, at $8 \mathrm{~cm}$ (Figure 4.3). In Figure 4.6, the photon interaction cross sections are shown in water. ${ }^{124}$ 
In the energy range of photons emitted by ${ }^{125}$, the likelihood of incoherent (Compton) scattering and the photoelectric effect are equivalent. However, for ${ }^{169} \mathrm{Yb}$ and ${ }^{192} \mathrm{Ir}$ (0.093 MeV and $0.38 \mathrm{MeV}$, respectively), it is clear that the Compton effect dominates, and the photoelectric effect probability decreases by several orders of magnitude.

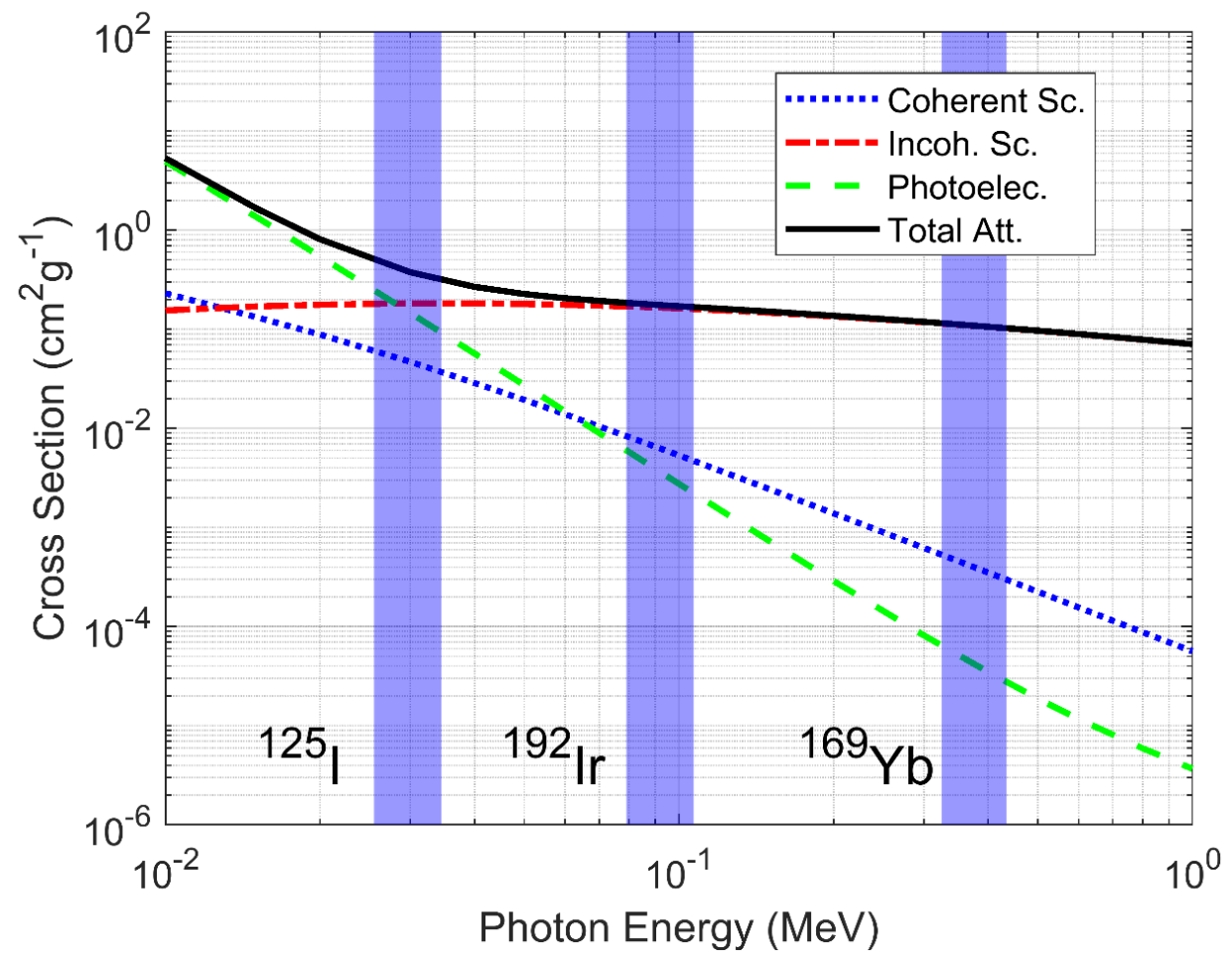

Figure 4.6: Photon interaction cross sections in water obtained from the NIST XCOM database. ${ }^{124}$ The average energy of the three radioisotopes examined is highlighted. Pair production not shown due to zero probability at the energy range shown.

This supports the explanation for why $\mathrm{RBE}_{\mathrm{M}}$ increases in ${ }^{169} \mathrm{Yb}$ and ${ }^{192} \mathrm{Ir}$ yet decreases slightly in ${ }^{125} \mathrm{I}$ as a function of distance from the source. For the two higher energy sources, the increased likelihood of the Compton effect results in increased 
scattered radiation further away from the source. Given the fact that scattered radiation is lower in energy and has higher LET, the increased $R_{B} E_{M}$ is to be expected. This effect is not observed in ${ }^{125}$ I because the likelihood of secondary photons being produced and reaching those distant voxels is significantly less. In order to corroborate this explanation, the electron and photon energy fluence spectra were obtained for all three sources at both the source position and at $8 \mathrm{~cm}$ away. As shown in Figure 4.4, both ${ }^{169} \mathrm{Yb}$ and ${ }^{192}$ Ir exhibit an increase in low energy photons and electrons, whereas this is not seen in the case of ${ }^{125} \mathrm{I}$. This can be explained by the greater probability of the photoelectric effect occurring, which results in the production of electrons that would only travel short distances in water $(<0.25 \mu \mathrm{m}) .{ }^{125}$ It is also important to note that $\mathrm{RBE}_{\mathrm{M}}$ increase exhibited by ${ }^{169} \mathrm{Yb}$ at the periphery (1.066) was greater than that of ${ }^{192} \mathrm{Ir}$ (1.030). While ${ }^{169} \mathrm{Yb}$ has been reported to have a higher relative biological effectiveness ${ }^{98,126,127}$ compared to ${ }^{192} \mathrm{Ir}$, the greater increase in $\mathrm{RBE}_{\mathrm{M}}$ exhibited at the periphery was unexpected. This result may be caused by the fact that the ${ }^{169} \mathrm{Yb}$ source has a more extensive lower energy component in comparison to ${ }^{192} \mathrm{Ir}$, as shown in Figures 4.1 and 4.4 .

The second part of this study involved examining the potential impact of this distance-based $\mathrm{RBE}$ variation on a prostate treatment plan. In Figure 4.5B, $\mathrm{RBE}_{\mathrm{M}}$ distribution for a single slice bisecting the PTV is shown; the electron fluence for the same slice is shown in Figure 4.5A. This is a relatively nominal change in $\mathrm{RBE}_{M}(+3.6 \%)$ and was most probably caused by the increased scattered radiation present in the distant voxels due to the high amount of lower energy scattered Compton photons which in turn emitted lower energy electrons. It is also important to note that this 
nominal increase only occurred in the low-dose regions, further reducing its significance.

The results indicate that $\mathrm{RBE}_{\mathrm{M}}$ changes primarily occur in the low-dose region. The magnitude of increases are most likely not clinically significant. Rava et al. ${ }^{88}$ conducted a study in which electronic brachytherapy (EBT) was compared to ${ }^{192} \mathrm{Ir}$ brachytherapy using the concept of biologically equivalent dose. In their study, treatment plans were created for endometrial cancer patients using PLATO treatment planning software and then evaluated using physical dose and biologically equivalent dose (BED). By accounting for the low energy photons present in EBT using BED, they were able to achieve a decrease in bladder and rectal toxicity. Our study went one step further (examining RBE) with an ${ }^{192}$ Ir plan for prostate cancer.

There were several sources of uncertainty in this study. It is important to note that the $\mathrm{RBE}_{\mathrm{M}}$ trends observed in this study are limited to V79 Chinese hamster cells. ${ }^{67}$ Different cell lines would theoretically have a different gamma parameter, causing the calculated $\overline{\alpha / \beta}$ and resulting $\mathrm{RBE}_{\mathrm{M}}$ values to be different. Also, the technique is mainly valid at the low-dose limit (Section 4.3.3), although the general conclusions of the study should not be affected. The rationale for use in this study was potential organs at risk in brachytherapy are usually found outside of the high-dose regions. In Figures 4.2 and 4.3, the error bars represent the standard error on $\mathrm{RBE}_{M}$ values at each position calculated from multiple $(n=5)$ Monte Carlo simulations with different starting seed values for each iteration. As distance from the source increased, the uncertainty increased due to a reduction in the fluence of both photons and electrons due to attenuation in water. ${ }^{124}$ 


\subsection{Conclusion}

In this study, the nature of $\mathrm{RBE}_{\mathrm{M}}$ was examined for ${ }^{125 \mathrm{I}},{ }^{192} \mathrm{Ir}$, and ${ }^{169} \mathrm{Yb}$ as well as for a clinically utilized prostate treatment plan. Monte Carlo simulations were used to determine the electron energy fluence spectra produced at various positions around the source. Using this energy fluence and microdosimetric techniques, the $\mathrm{RBE}_{\mathrm{M}}$ at various positions was obtained. It was found that the $\mathrm{RBE}_{\mathrm{M}}$ of ${ }^{125} \mathrm{I}$ relative to ${ }^{192}$ Ir was 1.558 , consistent with previous works. The change in $\mathrm{RBE}_{\mathrm{M}}$ as a function of distance from the source was also examined. $\mathrm{RBE}_{\mathrm{M}}$ for ${ }^{125}$ I was found to decrease slightly to a value of 0.994 while it increased to approximately 1.030 and 1.066 for ${ }^{192} \mathrm{Ir}$ and ${ }^{169} \mathrm{Yb}$, respectively. The high dose rate ${ }^{192}$ Ir prostate treatment plan was found to exhibit $\mathrm{RBE}_{M}$ hot spots of $+3.6 \%$. To the best of our knowledge, these observations have not previously been reported in the literature. Future studies should include similar clinical treatment plan investigations for lower energy sources such as ${ }^{103} \mathrm{Pd}$ and ${ }^{125} \mathrm{I}$.

\subsection{Acknowledgements}

This work has been supported in part by a Discovery grant of the Natural Sciences and Engineering Research Council (NSERC; Grant No. RGPIN-435608). 


\section{Quantifying the Impact of Lead Doping on Plastic Scintillator Response to Radiation}

This Chapter is based on an original article which has been accepted for publication as:

H. Nusrat, G. Pang, S.B. Ahmad, B. Keller, A. Sarfehnia

"Quantifying the impact of lead doping on plastic scintillator response to radiation". (2019)

Medical Physics, [IN PRESS], DOI: 10.1002/mp.13691

\subsection{Abstract}

Purpose: Through the addition of high-Z dopants, the sensitivity of plastic scintillators to low-energy radiation can be increased. This study quantifies this change in sensitivity as a function of dopant concentration. Methods: Measurements were conducted using four different lead doped scintillators $(0 \%, 1 \%, 1.5 \%, 5 \% \mathrm{~Pb})$ in high energy electrons (6 to $15 \mathrm{MeV}$ ) and low energy photon (100 to $300 \mathrm{kVp}$ ) radiation fields. High-energy irradiations were done using a clinical linear accelerator, low energy irradiations were 
done using an orthovoltage unit. Light emitted by the scintillator was quantified using a photosensor module. The experimental set-up was replicated in Geant4.10.3 Monte Carlo and scintillator parameters (Quenching parameter: $k B$ and the light yield: $L_{0}$ ) were varied until agreement between measured and simulated results was reached. Monoenergetic electrons were used to simulate the high energy electron beam while a spectrum generated using SpekCalc® software was used in the low energy simulations. Light produced by the scintillator was quantified using a flux scorer sensitive only to photons in the visible wavelength range. In order to compare measured and simulated results, the light produced by the scintillator was normalized to the absorbed dose to water at the point of measurement. Results: At high lead dopant concentrations, the scintillator's sensitivity to the $100 \mathrm{kVp}$ beam increased by $474 \%$ relative to the $15 \mathrm{MeV}$ electron beam; the scintillator's $k B$ parameter increased from $0.126 \mathrm{~mm} / \mathrm{MeV}$ to 0.27 $\mathrm{mm} / \mathrm{MeV}$. A model quantifying the change in $k B$ and $L_{0}$ as a function of $Z_{\text {eff }}$ was derived; presenting a modified Birks' Law for metal doped plastic scintillators. Conclusion: The impact of high- $Z$ doping on plastic scintillator response was quantified; this can allow for the controlled induction of energy dependence in plastic scintillator detectors.

Keywords: scintillators, quenching, high-Z doping

\subsection{Introduction}

The popularity of plastic scintillators in radiation dosimetry has increased significantly in the recent past. Plastic scintillators, also referred to as organic scintillators, consist of one or multiple fluors dissolved in a hydrocarbon base. As 
particles of ionizing radiation traverse the plastic scintillator, they will experience energy loss; this energy then flows via non-radiative dipole-dipole interactions (Forster transfer) to an organic benzene ring-based molecule. The excitation and subsequent deexcitation of the organic molecule will result in the emission of an ultraviolet photon which is absorbed by a wavelength shifting phosphor. The excited phosphor molecule undergoes a Stokes' shift emission event, releasing a photon in the visible wavelength range.

Numerous studies have demonstrated that plastic scintillators are excellent detectors for use in clinical radiation dosimetry. ${ }^{1-11}$ Several factors contribute to their suitability. These include: 1) water equivalence, 2) linearity, and 3) fast response. The first study to examine the feasibility of plastic scintillator use in clinical radiotherapy was conducted by Beddar et al (1992), ${ }^{51,128}$ who proposed a novel detector design which comprised of a plastic scintillator coupled to a fiber optic cable which terminated at a photomultiplier tube. This study also attempted to quantify the impact of Cherenkov light produced, and its effect on the scintillator's signal. Since this work by Beddar et al., many different detector designs have been proposed.

When the response of plastic scintillators to high linear energy transfer (LET) radiation was examined, it was found that the amount of light produced was significantly less than predicted. Plastic scintillators were found to exhibit a definite 'quenching' effect when irradiated with high LET radiation such as protons, negative pions, low energy photons, and low energy electrons. ${ }^{138-141}$ This effect, formally known as 'ionization quenching', was first discovered by Birks in 1951 has since been studied in depth for various radiation beams. ${ }^{52}$ The contemporary explanation for ionization 
quenching physics is that high LET particles cause the production of reactive molecules which allow for energy to be dissipated, reducing the amount of energy expended through light emission. ${ }^{142}$

The response of a plastic scintillator can be modelled by the equation,

$$
\frac{d L}{d x}=L_{0} \frac{\frac{d E}{d x}}{1+k B\left(\frac{d E}{d x}\right)}
$$

In this equation, referred to as Birks' Law, ' $d L / d x$ ' represents the light intensity (or the number of visible wavelength photons) emitted by the scintillator per unit length, ' $L_{0}$ ' is the light yield per unit energy, ' $d E / d x$ ' is the scintillator's collisional stopping power, and ' $k B$ ' is the quenching parameter. According to Birks' Law, linearity between radiation energy deposited and light emitted by the scintillator is preserved until the collisional stopping power reaches a threshold value. Once the value of collisional stopping power increases enough, linearity is lost and the quenching (represented by the factor ' $k B$ ') becomes significant. In the case of electrons, the increased stopping power at lower electron energies does correspond to greater quenching effects.

The electron energy threshold at which this quenching effect begins to materialize has been widely disputed in the literature. Williamson et al. (1999), ${ }^{143}$ conducted a study with the aim of deducing the actual threshold below which quenching occurs. In this study, a plastic scintillator was exposed to various low energy photons and it was found that the relationship between energy deposited and scintillator response exhibited linearity at energies greater than $125 \mathrm{keV}$. However, the results obtained by Williamson et al., could not be replicated numerically using the unimolecular 
model of scintillator light production. Prior to this work, various studies argued the existence of different thresholds, reaching down to $20 \mathrm{keV} .{ }^{144}$

In order to enhance quenched scintillator response at lower energies (and high LETs), high-Z doping can be used. ${ }^{138,145-148}$ By doping with high-Z elements, the radiation interaction cross-section is changed, allowing the non-radiative energy dispersion (quenching) discussed previously to be compensated against through increased occurrence of the photoelectric effect. Additionally, the presence of high-Z elements increases the production of secondary electrons which act antagonistically to the quenching process. This means that the increase in light production due to the creation of increased secondary electrons compensates for the light reduction due to quenching. Due to the photoelectric effect dominating the photon interactions at low energies, and the strong dependence of photoelectric interaction cross-section on effective $Z$ of the medium, even the addition of a small amount of high- $Z$ dopants significantly increases low energy radiation sensitivity.

Previous works examining high-Z doped plastic scintillators have been limited. Hyman et al. (1958), ${ }^{149}$ examined the feasibility of various production methods for leadloaded plastic scintillators. In this pioneering study, it was suggested that high-Z doped scintillators would have applications in the detection of low energy $x$-ray and gamma radiation, as well as thermal neutron detection. Tsou (1965) ${ }^{150}$ conducted a study in which the methodology for the production of high-Z doped organic (plastic) scintillators was examined. This work determined that high-Z doping increased the sensitivity to low energy photons, although self-absorption due to doping increased. More recently, Britvich et al. $(2000)^{151}$, also explored optimal methods of producing doped plastic 
scintillators. In this work, the effect of dopant type on the emission wavelength and scintillator decay time was examined.

Thus far, a quantitative analysis examining the impact of different dopant concentrations on scintillator response at different energies has not been conducted. In order to further the use of high- $Z$ doped plastic scintillators, it is imperative that accurate values of $k B$ and $L_{0}$, the quenching parameter and the light yield seen in Birks' Law (Equation 5.1), be determined. In this study, the response of four different concentrations of lead-doped plastic scintillators was studied in order to systematically quantify the impact of high- $Z$ doping. A modified Birks' Law is presented which can accurately model the scintillator response of high- $Z$ doped plastic scintillators for a wide range of energies.

\subsection{Materials and Methods}

\subsubsection{Overall methodology}

In this work, response is defined as the amount of light emitted by the scintillator per unit absorbed dose-to-water. Differently doped scintillators were used to experimentally measure the response to radiation beams of various energies. A Monte Carlo simulation was used to accurately simulate the radiation beams and the experimental setup, and the scintillator response was numerically determined. The Birks' Law parameters $\left(k B\right.$ and $\left.L_{0}\right)$ were varied until the measured and simulated scintillator responses matched, thus revealing the impact of lead-doping on plastic 
scintillator response. For the purposes of this study, 'low energy' refers to electron energies less than $100 \mathrm{keV}$ at the point of measurement.

\subsubsection{Measurements}

Four different commercially available lead doped plastic scintillators were used. These included an undoped scintillator (0\% Pb; EJ200) as well as $1 \% \mathrm{~Pb}, 1.5 \% \mathrm{~Pb}$, and $5 \% \mathrm{~Pb}$ doped (EJ256) scintillators. These scintillators were purchased from Eljen Technology (Sweetwater, TX). Additionally, to ensure reproducibility, consistency, and robustness, lead-doped scintillators were also purchased from a different manufacturer and subjected to the same measurements. These included $0 \%$ (BC408), 1\%, and 5\% Pb-doped (BC-452) scintillators purchased from Saint-Gobain (Hiram, $\mathrm{OH}$ ).

For the measurements, in order to collect the light produced by these scintillators (emission wavelength centered primarily on $440 \mathrm{~nm}$ ), a flat-cleave optical fiber was coupled to the scintillator using a custom-built detector apparatus (Figure 5.1A). Through this detector apparatus, each scintillator (cylinders of $5 \mathrm{~mm}$ height and diameter) was directly coupled to the fiber one at a time. The detector apparatus was composed of polyether ether ketone (PEEK), surrounded by virtual water $(20 \times 20 \times 20$ $\mathrm{cm})$. The virtual water cap allowed for insertion into a clinically utilized slab of virtual water, facilitating radiation beam measurements to be done at $1.5 \mathrm{~cm}$ depth in water. The optical fiber was then coupled to the Hamamatsu H10721 photosensor module (operated at a constant gain of $5 \times 10^{5}$ ) and the charge produced during scintillator irradiation (250 cGy) was collected using an electrometer. 
In the measurements, 250 Monitor Units were delivered by each machine and absorbed dose-to-water was calculated. The AAPM Task Group 51 and Task Group 61 were used for reference dosimetry in high energy electron and orthovoltage beams, respectively. ${ }^{152,153}$ Orthovoltage $\mathrm{x}$-ray measurements were conducted using an open cone (10 cm diameter) at a source to surface distance (SSD) of $30 \mathrm{~cm}$ (Figure 5.1B). The electron beam measurements were conducted using a $10 \times 10 \mathrm{~cm}^{2}$ beam at an SSD of $100 \mathrm{~cm}$.

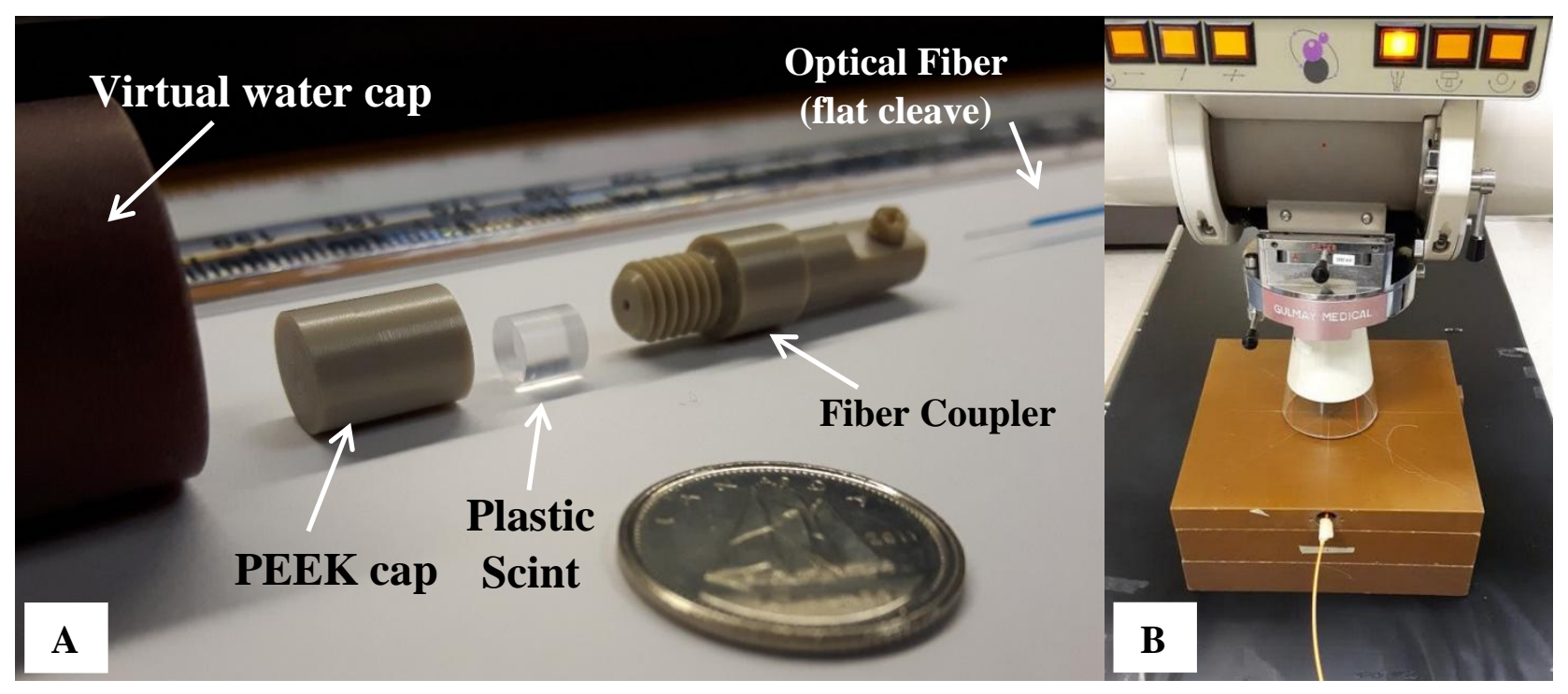

Figure 5.1: (A) Specialized detector housing (shown in grey). This allowed for the scintillator to be coupled to the flat-cleaved optical fiber for light collection. The virtual water cap (shown left) allows for insertion into clinically used virtual water slabs. The coin is shown for scale and has a diameter of $18.03 \mathrm{~mm}$. (B) Measurement set-up in the Orthovoltage x-ray unit; the scintillator detector was placed at a depth of $1.5 \mathrm{~cm}$ in water. 
Various radiation beams were used to irradiate the scintillators. The goal was to utilize high and low energy radiation beams, which would allow for the observation of quenching and how it was impacted by lead-doping in both energy ranges. The radiation beams used in this work were: $6,9,12$, and $15 \mathrm{MeV}$ electrons, as well as 100, 180,250 , and $300 \mathrm{kVp} \times$ rays. At the higher energies, electron beams were used instead of photon beams due to the relatively sharp spectra of a clinical electron beam, allowing for simpler beam matching, and more accurate modelling in the Monte Carlo step that followed. The high energy electron beams were produced using an Elekta Synergy clinical linear accelerator (Elekta AB, Stockholm, Sweden); the kVp x rays were produced using a Gulmay® D3000 Orthovoltage Unit (Surrey, UK). During measurements, it was ensured that the room lights were turned off and any optical interference or noise was minimized. Irradiations were repeated for each scintillator and beam to quantify measurement uncertainty $(n=5)$; measurements were repeated on different days and using the scintillators from the two different manufacturers to ensure experimental reproducibility. The lowest energy beam used (100 kVp $x$ rays) corresponded to the estimated scintillator quenching threshold described in the literature..$^{143,144}$

Cherenkov light produced in the optical fiber during the irradiation events was also quantified and subtracted. In order to determine the magnitude of the Cherenkov light signal for each beam, the enclosed detector apparatus without the scintillator (i.e. fiber only) was placed in the beam and the response was measured. The amount of Cherenkov light produced was $5.6 \%$ of the undoped scintillator light for the $15 \mathrm{MeV}$ electron beam, and $0.19 \%$ of the $300 \mathrm{kVp}$ orthovoltage beam. The 100 and $180 \mathrm{kVp}$ 
orthovoltage beams did not produce electrons with energies greater than the Cherenkov production threshold for the optical fiber used.

Absorbed dose to water was determined by the AAPM TG-51 and TG-61 reports for electron and orthovoltage beams, respectively. ${ }^{152,153} 250$ Monitor Units were delivered in all machines and the corresponding absorbed doses were calculated.

\subsubsection{Monte Carlo simulation}

Monte Carlo simulations were used to replicate the measurement conditions described. Geant4.10.3 ${ }^{108}$, a Monte Carlo tool-kit, was used to simulate radiation transport through the scintillator and the surrounding materials (water, PEEK).

To model the electron beams generated by the linear accelerator, monoenergetic electrons were used in the simulation. In order to determine the x-ray spectrum to input into the simulation, the SpekCalc® software package was used to determine the x-ray spectra given the tube voltage and filtration conditions. ${ }^{14}$ In the simulation, the low energy physics list (EMStandardPhysics_Option4) was used. The electron, positron, and photon range cut-off was set to $100 \mathrm{~nm}$; this was equivalent to an electron energy cut-off threshold of approximately $100 \mathrm{eV}$. The physics processes used in the simulation were validated against published literature: scintillation physics processes were

validated using the scintillator response shown by Beddar et al. (2009), ${ }^{154}$ and electron transport was validated against data from Rogers and Bielajew (1986). ${ }^{155}$ The SpekCalc ${ }^{\circledR}$ generated orthovoltage spectra were validated against measured depth dose-curves in water. 
In order to numerically evaluate scintillator response, the optical physics package in Geant4 was enabled and Birks' Law was used to determine the total number of scintillation photons produced by the scintillator due to irradiation by a particular beam. The optical physics package ${ }^{110}$ in Geant4 allows for modelling visible photon transport and production via scintillation and Cherenkov processes. The optical physics package allows for modelling of scintillator light production using Birks' Law; photons are calculated per G4Step and correspond to the electron's stopping power and energy deposited. ${ }^{156} \quad$ Scintillation photons were counted using a command-based flux scorer surrounding the scintillating volume. Simulations were run for $10^{9}$ initial particle histories, corresponding to a dose (to water) uncertainty of $0.05 \%$, and a scintillator light uncertainty of $0.10 \%$. During each simulation, the electron energy spectrum within each scintillator was also scored. For each beam, the energy spectrum of electrons produced was used to obtain the mean electron energy at the point of measurement.

Scintillator responses were also presented as a function of collisional stopping power. Values were obtained by matching the mean electron energy to its corresponding stopping power using the NIST ESTAR database ${ }^{157}$; this method has been previously described by Granville and Sawakuchi. ${ }^{158}$

\subsubsection{Scintillator Response Analysis}

To compare simulation to measurements, the amount of scintillator light produced was normalized to absorbed dose in all cases. This removed the impact of factors such as output variations, irradiation time, and initial number of simulated 
particles in the comparison. In the simulation, the experimental conditions described in Section 5.3.2 were replicated. In order to connect the measured and simulated responses, dose to water in the simulation at the point of measurement was obtained by converting all non-water heterogeneities to water material. It is important to note that the two simulations (scintillator assembly and water only) were run using the same physics processes and the same number of primary particles. Mean electron energies were obtained by collecting the electron energy fluence spectra at the volume of the scintillator using Geant4 Monte Carlo and calculating weighted averages. Mean collisional stopping powers were obtained using the same technique after converting spectra from electron energy to collisional stopping power as described in Section 5.3.3.

In order to quantify the impact of inorganic high- $Z$ doping on the response of plastic scintillators, an equation was obtained by curve fitting the $k B$ and $L_{0}$ data (Equations 5.2 and 5.3).

\subsection{Results}

\subsubsection{Lead Doping and Measured Response}

An analysis of the uncertainties associated with the measurement results is shown in Table 5.1. Given the scintillator responses were normalized against absorbed dose at the point of measurement, the overall uncertainties include scintillator and machine output dependent factors which were added in quadrature assuming all uncertainty sources are independent of one another. Table 5.1 is divided into two sections: 1) uncertainties associated with the scintillator measurement and, 2) 
uncertainties associated with machine output variation. In the scintillator measurement section, the 'standard error of the mean' and the 'Cherenkov effect' were determined through repeat measurements $(n=5)$. The 'standard error of the mean' of measurements includes all uncertainties associated with imperfect and varying contact between the scintillator and the optical fiber as well as any potential photodetector response variation. Uncertainties due to manufacturing variation were estimated based on the measurements from the scintillators purchased from two different manufacturers (further explained in Section 5.3.2); company variations were found to be minimal (Manufacturer variation in Table 5.1). Regarding positioning setup uncertainties, there was no statistically significant uncertainty in depth for the scintillators because of the use of a solid piece of virtual water phantom for this purpose. The uncertainties on machine output variation for linac and orthovoltage measurements were estimated using data from AAPM's TG-51 and its addendum, as well as TG-61 Protocols respectively. ${ }^{152,159,160}$ Table 5.1 uses identical notations/terminology for output measurement as those from the task groups. In the uncertainty analysis, the 'standard error of mean', 'Cherenkov effect', 'manufacturer variation', and 'measurement reproducibility' were type A uncertainties (determined through repeated measurements), whereas all other uncertainties listed were type B. ${ }^{161}$ 
Table 5.1: Measurement Uncertainty Analysis. In the 'Output Measurement' section of the uncertainty analysis, '*' is used to reference the TG-51 protocol ${ }^{152}, 159$ while “**' is used to reference the TG-61 protocol. ${ }^{162}$ All uncertainty components given as relative standard deviations $(k=1)$.

\begin{tabular}{|c|c|c|}
\hline & $\begin{array}{l}\text { Linac } \\
\text { Electrons } \\
(\%)\end{array}$ & $\begin{array}{l}\text { Orthovoltage } \\
(\%)\end{array}$ \\
\hline \multicolumn{3}{|l|}{ Scintillator Measurement } \\
\hline $\begin{array}{l}\text { Reproducibility of Scintillator } \\
\text { Measurement }(n=5)\end{array}$ & 0.048 & 0.45 \\
\hline Cherenkov effect & 0.071 & 0.28 \\
\hline Manufacturer variation & 0.20 & 0.56 \\
\hline \multicolumn{3}{|l|}{ Positioning Setup } \\
\hline SSD variation & 0.39 & 1.3 \\
\hline Lateral variation & 0.3 & 0.3 \\
\hline In-phantom depth & 0 & 0 \\
\hline \multicolumn{3}{|l|}{ Output Measurement } \\
\hline Measurement reproducibility & 0.31 & 0.42 \\
\hline \multicolumn{3}{|l|}{ Other } \\
\hline $\begin{array}{l}P_{\text {tp }}, P_{\text {poll }}, P_{\text {ion }}\left(\text { Water }^{\star}\right) \\
P_{\text {tp }}, P_{\text {pol }}, P_{\text {ion }}, P_{\text {stem }}\left(\text { air }^{* \star}\right)\end{array}$ & 0.35 & 1.0 \\
\hline Calibration factor & 0.5 & \\
\hline $\begin{array}{l}N_{D, w}{ }^{*} \\
N_{k}{ }^{* *}\end{array}$ & & 0.4 \\
\hline Effect of beam quality & 0.2 & 2.0 \\
\hline
\end{tabular}




\begin{tabular}{|lll|}
\hline $\begin{array}{l}\text { difference } \\
\text { calibration } \\
\text { measurement }\end{array}$ & $\begin{array}{c}\text { between } \\
\text { and }\end{array}$ & \\
\hline $\begin{array}{l}\mathrm{K}_{\mathrm{e}, \text { cal }}{ }^{*} \\
\mathrm{~B}_{\mathrm{w}}\left(\mu_{\mathrm{en}} / \rho\right)^{* *}\end{array}$ & 1.3 & 2.2 \\
\hline $\begin{array}{l}\text { Positioning } \\
\begin{array}{l}\text { Output variation } \\
\text { measurements }\end{array}\end{array}$ & 0.49 & 1.8 \\
\hline Combined Uncertainty (k=1) & 0.2 & 0.3 \\
\hline
\end{tabular}

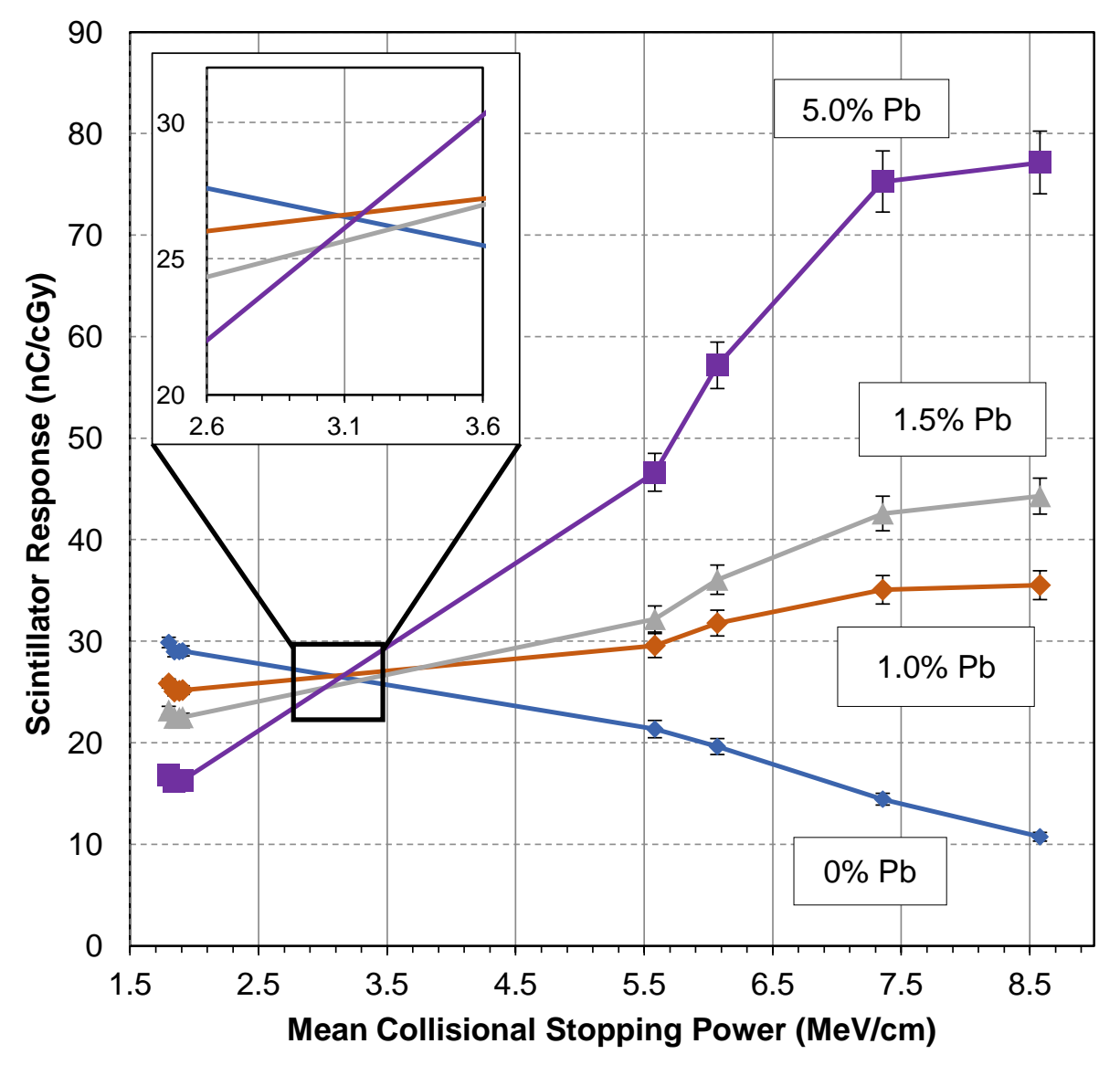

Figure 5.2: Measured scintillator response. The light signal as read by PMT (charge, $\mathrm{nC}$ ) normalized to absorbed dose to water at point of measurement (at $1.5 \mathrm{~cm}$ depth for each respective beam). Response is shown as a function of mean collisional stopping power determined using the NIST ESTAR database. ${ }^{125}$ The subplot is showing an 
enlarged representation of the response to low stopping power (electron beam) radiation at the point where the impact of doping becomes insignificant. The mean collisional stopping power shown on the x-axis was obtained as explained in Section 5.3.3.

In figures 5.2 and 5.4 , the error bars represent the total uncertainty on the measured dose-normalized scintillator response (Table 5.1). In Figure 5.2, the dosenormalized scintillator response is shown as a function of mean collisional stopping power. The light generated by each scintillator for various energies was normalized to absorbed dose to water at the scintillator's position $(1.5 \mathrm{~cm}$ depth in water). With mean electron collisional stopping powers less than $3.15 \mathrm{MeV} / \mathrm{cm}$, the increased doping concentration resulted in a decreased signal while at greater mean electron collisional stopping powers, the opposite was observed.

\subsubsection{Birks' Law Parameter Optimization}

The scintillator dependent parameters of Birks' Law were optimized in the Monte Carlo simulation until simulated and measured responses agreed (Figure 5.3). The light yield $\left(\mathrm{L}_{0}\right)$ values for the $0 \%, 1.5 \% \mathrm{~Pb}$-doped, and the $5.0 \% \mathrm{~Pb}$-doped scintillators were previously published by the manufacturer (Eljen Technologies). ${ }^{163}$ The measured values of $L_{0}$ were determined using the methods traditionally used for absolute light yield determination as described by de Haas et al., 2004. ${ }^{164}$ The $1.0 \%$ Pb-doped light yield was determined using linear interpolation (Figure 5.3) due to the relatively linear 
relationship between doping concentration and scintillator transparency. Thus, a relation between $L_{0}$ and the effective atomic number $\left(Z_{\text {eff }}\right)$ based on the four differently doped scintillators used was determined,

$$
L_{0}\left(Z_{e f f}\right)=-1173.1\left[Z_{e f f}\right]+16041 .
$$

Equation 5.3 represents the relation which was obtained by optimizing the Birks' quenching parameter $(k B)$ for the four experimentally tested differently doped plastic scintillators. In this equation, the effective atomic number $\left(Z_{\text {eff }}\right)$ is the average atomic number of the scintillator compound. The $k B$ values presented are the Geant4 simulation parameters optimized to yield closest agreement between simulated and measured scintillator responses. Once again, lead doped scintillators were obtained from two different companies, ensuring that measured responses were accurate and company variations between scintillators was minimal ('Manufacturer variation' in Table 5.1). It is important to note that the Birks' parameter was not varied as a function of energy, only as a function of doping concentration. Equations 5.2 and 5.3 are valid for $Z_{\text {eff }}$ values ranging from 5.5 to 10 . The optimal $\mathrm{kB}$ and $\mathrm{L}_{0}$ values used to determine the $Z_{\text {eff }}$ dependence are shown in Figure 5.3. This is further explained in the discussion.

$$
k B\left(Z_{\text {eff }}\right)=\frac{-0.00944\left[Z_{e f f}\right]^{2}+0.4578\left[Z_{e f f}\right]-2.103}{Z_{e f f}-4.344}
$$




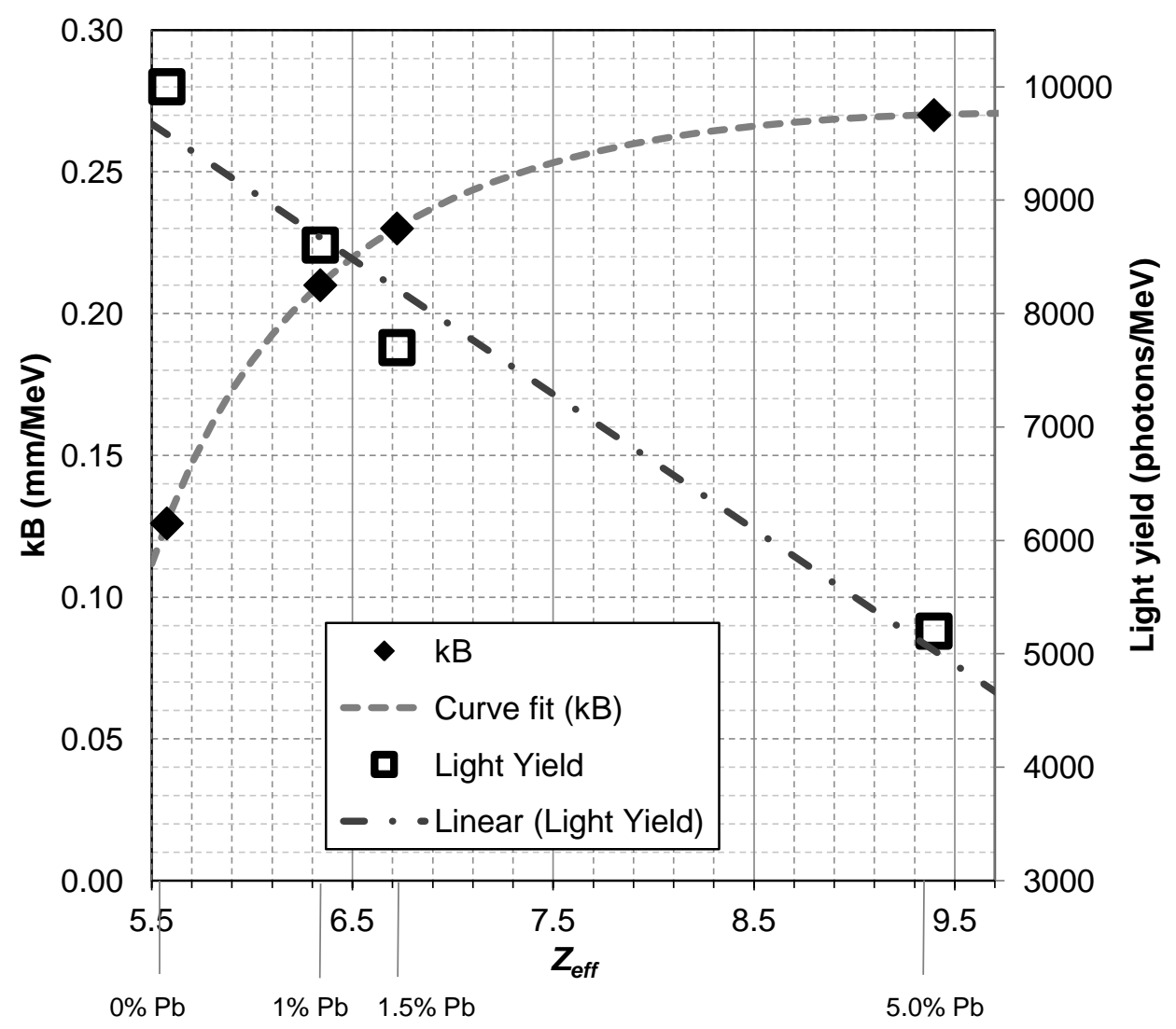

Figure 5.3: Optimal kB values that result in agreement between Monte Carlo scintillation and measurement results. The scintillator light yield $\left(\mathrm{L}_{0}\right)$ used in the simulation is also shown in this figure (secondary $\mathrm{y}$-axis). $\mathrm{kB}$ values are presented using units of $\mathrm{mm} / \mathrm{MeV}$, while light yield is shown in photon/MeV; both sets of data are shown as a function of the effective atomic number of each scintillator. The 'kB Curve Fit' values are also shown as a function of effective $Z$ of the scintillator material; curve fitting of the data was done using MATLAB. The corresponding lead doping percentages are shown below the $\mathrm{x}$-axis. 


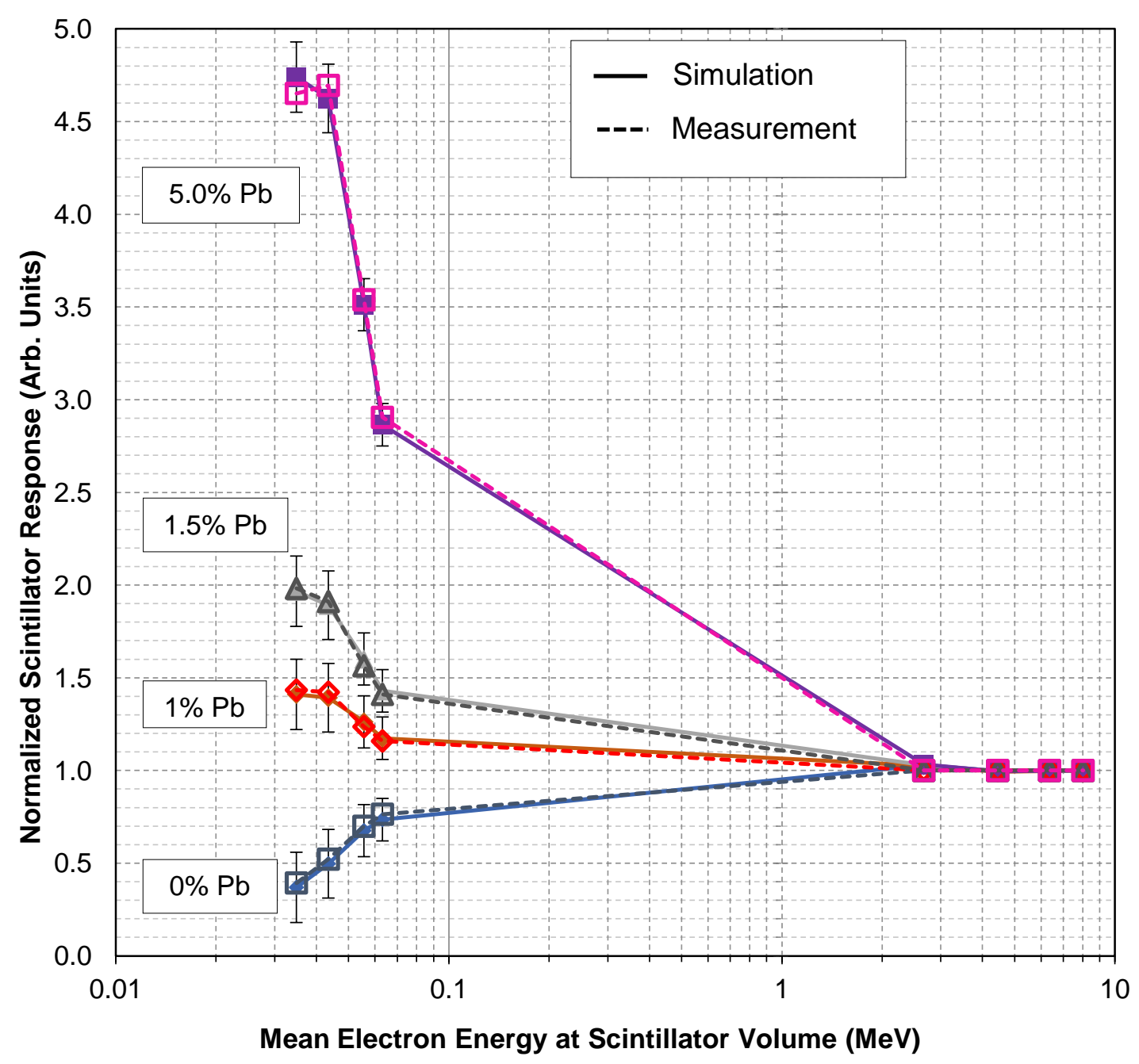

Figure 5.4: Measured response of the differently lead-doped scintillators to mean energy of electrons in the scintillator volume. For each scintillator, its response at a given energy is normalized to its response to the $15 \mathrm{MeV}$ electron beam (average electron energy at $1.5 \mathrm{~cm}$ was approximately $8 \mathrm{MeV})$. Measured results are shown as solid lines, while simulated results use dotted lines. All measured data shown was obtained using the scintillators obtained from Eljen Tech., although two different manufacturers were tested to ensure reproducibility (Section 5.3.2). 
In Figure 5.4, all scintillator responses were normalized to the response at 15 MeV. This allowed for clarity in observing the response enhancement at lower energies. It is important to reiterate that the quantity 'scintillator response' refers to the visible light produced by the scintillator normalized to the absorbed dose to water at the point of measurement (i.e., with the detector being replaced by water). In this figure, the measured and simulated responses of the four different lead doped scintillators examined are presented. As explained in Section 5.3.4, the x-axis corresponds to the mean energy of electrons present at the scintillator under each beam. The $y$-axis corresponds to the light signal normalized to the absorbed dose-to-water at the volume of measurement for each scintillator. Figure 5.4 also demonstrates the results of the Monte Carlo modelling of doped scintillator response with optimized parameters discussed in Figure 5.3.

\subsection{Discussion}

The findings of this study quantify the increased sensitivity of high- $Z$ doped plastic scintillators to lower energy $\mathrm{x}$ rays and electrons. As shown in Figures 5.2 and 5.4 , exposure to lower energy $x$ rays caused the amount of light produced by the leaddoped scintillators to be greater.

The results shown in Figure 5.2 demonstrate the scintillator's response to the high energy electron and orthovoltage $x$-ray beams. The responses shown are the photodetector charge collected $(\mathrm{nC})$ normalized to absorbed dose to water at the effective point of measurement. While the $5 \%$ lead doped scintillator exhibited a 
significant increase in light emission at the lower energies, the response to the higher energy $(>1 \mathrm{MeV}$ ) radiation beams was reduced relative to the undoped case. This observation may be explained by the fact that the addition of lead reduces the transparency of the scintillator, increasing self-absorption. The subplot in Figure 5.2 shows the point at which the impact of lead on scintillator response becomes insignificant (high energy, low LET radiation). This occurs at approximately $3.1 \mathrm{MeV} / \mathrm{cm}$. In Figure 5.4, the response of each scintillator was normalized to its own response at the highest energy $(15 \mathrm{MeV})$. This higher energy normalization was done to be able to interpret the impact of doping with increased clarity. At higher electron energies, the collisional stopping power does not change significantly. ${ }^{125}$ As shown in Figures 5.2 and 5.3, this translates to a relatively constant scintillator response due to the stopping power dependence of Birks' Law.

As shown in Figure 5.4, the impact of lead doping increased the low energy sensitivity of the scintillator significantly. When the undoped scintillator is considered, the use of lower energy radiation resulted in a reduction in response; the response produced at $100 \mathrm{kVp}$ was only $37 \%$ of the $15 \mathrm{MeV}$ response. Measurements with the lead doped scintillators demonstrated the opposite. In the $1.0 \% \mathrm{~Pb}$ doped scintillator, the $100 \mathrm{kVp}$ response was $141 \%$ of the $15 \mathrm{MeV}$ response. Similarly, a $195 \%$ and a $474 \%$ increase were seen when comparing $100 \mathrm{kVp}$ to $15 \mathrm{MeV}$ for the $1.5 \%$ and the $5.0 \% \mathrm{~Pb}$-doped scintillators, respectively. The reduction in response observed in the undoped scintillator occurred due to scintillator quenching. At lower energies, not all molecular excitations resulted in the emission of a visible photon. By adding lead, the 
probability of the photoelectric effect increased which resulted in an increase in energy absorbed in the scintillator.

The results of the simulated scintillator responses are shown in Figure 5.4. Using Geant4.10.3, each unique scintillator was modelled and irradiated with a replica of the beams used experimentally. Birks' law was used by the software in order to predict the number of photons emitted; in order to achieve agreement between the measured and simulated responses, the Birks constant, kB, was varied (Figure 5.3). As doping concentration increased, the observed trend was that the value of $k B$ also increased. As seen in Figure 5.3, this increase was rapid between 0 and $1.0 \% \mathrm{~Pb}$ doping and saturated at higher doping concentrations (5.0\% doping; $\left.Z_{\text {eff }}=9.4\right)$.

It is important to comment on the universality of equations 5.2 and 5.3. Although the measurements and data presented has been collected for lead doping, generalizing the function of effective $Z$ should be possible and accurate because it is the higher atomic number of the dopant which causes the change in response, nothing that is limited to lead only. The presence of lead within the scintillator volume causes a shift in the radiation interaction cross sections towards the photoelectric effect. The magnitude of this phenomena increases monotonically as the dopant's atomic number increases. The proposed bounds on equations 5.2 and 5.3 are $Z_{\text {eff }}$ ranging from 5.5 to 10 . Organic scintillating materials with an effective atomic number less than 5.575 do not exist. ${ }^{125}$ Similarly, while doping the scintillator to very high concentrations is theoretically possible, the increased amounts of dopants would render the scintillator useless due to diminished transparency. 
The largest deviation between the measurement and simulation occurred in the response of the $5.0 \% \mathrm{~Pb}$-doped scintillator. Measured results indicated that the response to $100 \mathrm{kVp} x$ rays was slightly greater $(+11 \%)$ than the response to $180 \mathrm{kV} \mathrm{x}$ rays (Figure 5.4). However, the simulation found that the $180 \mathrm{kVp} \times$ rays caused a slightly greater response (approximately 4\%) when compared to the $100 \mathrm{kVp} \times$ rays. From measurements, it was expected that the difference in response for these two energies would be minimal. This difference may be due to uncertainties with the 100 $k V p$ x-ray spectrum generated using SpekCalc $\circledast{ }^{14}$ Any minute deviations from the Orthovoltage machine's 'true' beam spectrum would most likely have a detectable impact at the lowest energy $(100 \mathrm{kVp})$ and highest doping concentration (5\% Pb). It is also important to note that in this work, $k B$, was not varied as a function of energy. Although $k B$ has been shown to be energy dependent, its significance above an energy of $100 \mathrm{keV}$ has been proven negligible. ${ }^{165}$ Another possible cause for this discrepancy is the large size of the scintillator volume. As discussed in the Methods section, each scintillator is a cylinder measuring $5 \mathrm{~mm}$ in height and $5 \mathrm{~mm}$ in diameter. At lower energies and increased doping concentrations, volume averaging effects play a greater role. Reproducibility was ensured by conducting measurements from two different scintillator manufacturers (Section 5.3.2). Both sets of detectors generated light the same light signals $(0.20 \%$ variation for electrons; $0.56 \%$ variation for orthovoltage $x$ rays).

The body of literature discussing high- $Z$ doped scintillators is thus far limited to organometallic-doped scintillator synthesis studies. ${ }^{149,150,166}$ In 1999, Britvich et al demonstrated a novel chemistry methodology for the synthesis of organometallic doped 
plastic scintillators. ${ }^{151}$ In this work, it was demonstrated that for a given radiation beam, increasing organometallic dopant concentration results in a decreased absolute light output. While the base scintillator used (polystyrene) was different from the one used in this work (polyvinyl toluene), the decrease in light yield reported is consistent with our findings. Britvich et al reported a light yield decrease of $20 \%$ when comparing the $0 \%$ and $5 \%$ doped scintillators whereas our findings indicate a decrease of $48 \%$. This discrepancy can be explained by the difference in base scintillators, dopants, and doping methodology.

\subsection{Conclusions}

The goal of this study was to quantify the impact of high- $Z$ doping on plastic scintillator response to various radiation beams ranging from low to high energies. This work presents the first characterization of metal doped scintillator response. By determining equations for the quenching parameter $(k B)$ and the light yield $\left(L_{0}\right)$ as a function of the effective atomic number, this work presents a modified Birks' Law that is valid for different high- $Z$ doping concentrations over a wide energy range. By understanding doped scintillator response, multiple differently doped scintillators can be used to create a uniquely energy dependent detector array allowing for the unfolding of beam spectra. This can have applications in the medical, nuclear, and space radiation fields. Future directions include expanding the range of energies and dopants, as well as studying the doping effect in medium LET beams. 


\subsection{Acknowledgements}

The authors would like to acknowledge Karl Kromer and Harry Easton for their efforts in the fabrication of the detector set-up. This work has been supported in part by Discovery grants of the Natural Sciences and Engineering Research Council (NSERC; Grant No. RGPIN-435608 and RGPIN 311780-13). 


\section{Novel Method for LET Measurement in Low Energy Photon Beams using Doped Plastic Scintillators: Proof of Principle}

This Chapter is based on an article which is currently in preparation:

H. Nusrat, G. Pang, M. Paudel, A. Sarfehnia

"Novel method for LET measurement in low energy photon beams using doped plastic scintillators: proof of principle".

Medical Physics, [IN PREP]

\subsection{Abstract}

Purpose: With the advent of novel and more complex radiation treatment modalities, treatment planning with absorbed dose only is resulting in sub-optimal treatment. Accounting for biology-based quantities, such as the LET, will result in better treatment. In order for this to occur, clinically suitable LET detectors must be developed; the purpose of this work present a proof-of-principle for a device and technique which could measure LET. Methods: The light signal generated by a plastic scintillator $(\mathbf{M})$ is the 
product of the fluence of electrons traversing its volume $(\boldsymbol{\varphi})$ and the scintillator's 'response I. In this work, found differently doped plastic scintillators were used. Doping allowed for each scintillator to be uniquely energy-dependent, allowing for the LET fluence spectra to be resolved through $\varphi=R^{-1} S$. Measurements were conducted on four different $\mathrm{x}$-ray beams including $100,180,250$, and $300 \mathrm{kVp} x$-rays, as well as Iridium-192. The response includes the light generated by the scintillator, photon attenuation, and signal losses in the optical fiber, junction, and photodetector. In order to increase sensitivity to high LET electrons, exponentially increasing bins in the energy domain were chosen. Mean LET values were compared to Monte Carlo generated electron fluence spectra. Results: For each beam, the electron LET fluence was resolved using 4 bins. Mean LET values measured were 1.18, 1.10, 1.17, 1.12, and $0.47 \mathrm{keV} / \mu \mathrm{m}$ for the $100,180,250,300$ and Iridium-192 beams, respectively. The Monte Carlo predicted mean LET values were 1.72, 1.20, 0.99, 1.00, $0.53 \mathrm{keV} / \mu \mathrm{m}$ for the beams in the order previously listed. Conclusion: This method presents a new method of LET measurement in real time; increasing the number of scintillators will result in improved accuracy.

\subsection{Introduction}

Due to its inherently dangerous nature, it is important to deliver the correct amount of radiation to patients during cancer radiotherapy. The amount to be delivered is quantified using 'absorbed dose', a physics-based quantity which is equal to the amount of radiation energy absorbed per unit mass in tissue. Complex treatment plans 
are created based on maximizing the amount of absorbed dose to the tumour while minimizing it to healthy tissue. Additionally, most of the physics quality assurance testing done in radiotherapy is to ensure that the planned absorbed dose is indeed equal to the measured absorbed dose at a given point. Current clinical practice is based upon the linear quadratic model, which predicts that absorbed dose correlates well with cell death for the particle types and energies found in most hospitals. However, it is the clinical outcome, not the absorbed dose distribution, which is of utmost importance. As such, in addition to absorbed dose, considering the quality of the radiation beam and its energy deposition along with the radiobiology may lead to better predictions of clinical outcome.

Furthermore, the relation between absorbed dose and cell death may not hold as strongly in more recently developed treatment modalities. For example, in particle therapy (with medium and high LET radiation) and even in highly modulated photon treatments, the correlation between absorbed dose and cell death has been shown to loosen. Consider, for example, proton therapy: in order to account for the increased cellkilling efficiency of a proton beam, a weighting factor of 1.1 is used during treatment planning. The purpose of this weighting factor is to link proton cell death to that caused by Cobalt-60 gamma rays; however, this weighting factor has been shown to vary between 0.92 and 2.0 depending on the position relative to the Bragg peak. ${ }^{167}$ Similar examples can be found for other particle beams including carbon-ions and neutrons. $^{23,168-170}$ In photon therapy, recent moves towards highly modulated and conformal treatments means an increased presence of scattered, low energy radiation. 
This, in turn has been shown to cause more cell death than predicted by absorbed dose alone, resulting in increased out-of-field RBE. ${ }^{19,22,59,60,62,171}$

Typically, RBE is used to quantify the efficacy of a given type of radiation at killing cells. It is defined as the amount of absorbed dose required to achieve a biological endpoint (e.g. 10\% cell survival) normalized to the absorbed dose required by a reference radiation (usually $125 \mathrm{kVp}$ x-rays) to reach the same endpoint. This quantity has been shown to strongly depend on the linear energy transfer of radiation. Linear energy transfer, or LET, is the amount of energy absorbed per unit length in tissue along the particle's path. As the LET increases, the frequency of energy deposition events also increases causing irreparable DNA damage to occur. LET varies by particle type and energy (Table 6.1), and there is a strong desire for LET-based treatment planning, especially in the particle therapy community. ${ }^{29,54,78,172}$ In order to accurately incorporate LET into treatment planning, LET detectors must be available for sufficient quality assurance protocols. While others have previously worked on LET measurement ${ }^{173,174}$, there is no such clinically suitable detector available on the market today.

Table 6.1: Typical LET values for different types of radiation. ${ }^{175}$

\begin{tabular}{|c|c|}
\hline Radiation Type & LET (keV/ $\mathbf{\mu m})$ \\
\hline Co-60 & 0.3 \\
\hline 250 kVp x-ray & 2 \\
\hline 10 keV electron & 2.3 \\
\hline
\end{tabular}




\begin{tabular}{|c|c|}
\hline 1 keV electron & 12.3 \\
\hline $14 \mathrm{MeV}$ neutron & 12 \\
\hline Heavy charged particles & $100-200$ \\
\hline
\end{tabular}

In this work, a novel technique for resolving the electron LET spectrum. As a proof of principle, we use a prototype detector based on the proposed technique to measure the LET of several orthovoltage beams as well as an HDR ${ }^{192}$ Ir brachytherapy source. In this work, the sensitive detector volume based on the plastic scintillator, an organic material which emits visible light when ionizing radiation traverses through it. ${ }^{51}$ Specifically, a combination of lead doped plastic scintillators were used such that each detector had a unique energy response. The response of plastic scintillators can be predicted using Birks' Law, ${ }^{142,146}$

$$
\frac{\mathrm{d} L}{\mathrm{~d} x}=L_{0} \frac{\frac{\mathrm{d} E}{\mathrm{~d} x}}{1+k B\left(\frac{\mathrm{d} E}{\mathrm{~d} x}\right)}
$$

where $d L / d x$ is the differential amount of light emitted per unit length in the scintillating volume, $L_{0}$ is the scintillation efficiency, $k$ and $B$ are scintillator dependent constants which account for light quenching effects observed with high LET radiation. In the literature, these constants are grouped together and presented as ' $k B$ '. The scintillator's response is directly dependent upon the collisional stopping power $(\mathrm{d} E / \mathrm{d} x)$ of radiation passing through it. Doping the plastic scintillator with high- $Z$ elements causes the photon interaction cross sections to shift, increasing the secondary electron production within the scintillator. 
By using an array of these uniquely energy dependent detectors, the electron LET spectra, and resulting average LET, for five low energy photon beams were obtained in this work. In this paper, a proof-of-principle for the technique and detector design is presented.

\subsection{Materials and Methods}

\subsubsection{General Formalism}

The signal measured from a given detector is related to: 1) the response of that detector, and 2) the fluence of particles traversing its sensitive volume. In the case of plastic scintillators, the charge generated by a photosensor coupled to the scintillator surface is the measured signal, $\mathbf{M}$. The response $\mathbf{R}$ of the plastic scintillator is predicted by Birks' Law (Equation 6.1), however, the overall detector response of the system (including the scintillator, fibers, and read-out setup) also depend on factors such as light lost in the fiber and at various junctions, as well as photon attenuation. $\varphi$ is the electron LET fluence in the scintillator volume caused by a given beam.

Plastic scintillators are intrinsically dependent upon the collisional stopping power of radiation; as the stopping power increases, the amount of light produced increases up to a saturation threshold (i.e. quenching, Equation 6.1). When considering electrons at energies used in radiotherapy applications, the collisional stopping power is essentially equivalent to the unrestricted linear energy transfer. ${ }^{5,176}$ Given this fact, the response and the fluence of radiation passing through will henceforth be defined in terms of electron linear energy transfer (LET), represented by $L_{\infty}$. Given the notations 
introduced earlier, the relation between the measured signal, electron LET fluence, and light production within the scintillator can thus be described by,

$$
\boldsymbol{M}=\int_{L_{\infty}^{\min }}^{L_{\max }^{\max }} \boldsymbol{R}\left(L_{\infty}\right) \times \boldsymbol{\varphi}\left(L_{\infty}\right) d L_{\infty}
$$

According to Equation 6.2, by integrating the product of the detector energy response $\boldsymbol{R}$ and the electron LET fluence over the full range of electron LET spectrum present inside the detector (where 'min' is minimum LET and 'max' is maximum LET), yields the measured signal, $\boldsymbol{M}$. In reality, several corrections for the photosensor light yield, scintillator coupling efficiency, and photon to current conversions exist. However, this method relies only on the proportionality of $\boldsymbol{M}$ to fluence and response due to the fact the relative measured signals are used to obtain $\varphi\left(L_{\infty}\right)$. It is noteworthy that independence from the various factors listed above lend significant strength and robustness to this technique for spectrum resolution.

Through the measurement of only one scintillator, the LET fluence cannot be resolved. This changes if multiple scintillators with different responses are used in conjunction with the first, under the same beam. By using multiple differently doped scintillators, we have the following,

$$
\boldsymbol{M}_{\boldsymbol{i}}=\int_{L_{\infty}^{\min }}^{L_{\infty}^{\max }} \boldsymbol{R}_{\boldsymbol{i}}\left(L_{\infty}\right) \times \boldsymbol{\varphi}\left(L_{\infty}\right) d L_{\infty}
$$

where $I$, represents a different uniquely doped and thus energy-dependent scintillator. Given that the densities of all differently doped scintillators are within $5 \%$ of one another $^{49,177}$, the LET of electrons traversing the scintillator do not change significantly, 
allowing the relative $\boldsymbol{\varphi}\left(L_{\infty}\right)$ to be conserved for a given beam. It is important to note that at this point, the formalism will be presented in a general form - this technique can be conducted with many different detectors as long as they are uniquely energy dependent. The resolution of the unfolded spectrum is directly related to the number of uniquely energy dependent detectors used.

In order to extract the electron LET fluence spectrum, Equation 6.3 must be rewritten by divided into multiple parts. The signal measured by a given scintillator is thus,

$$
\begin{aligned}
\boldsymbol{M}_{\boldsymbol{i}}= & \int_{L_{\infty}^{\min }}^{L_{\infty}^{1}} \boldsymbol{\varphi}\left(L_{\infty}\right) \boldsymbol{R}_{\boldsymbol{A}}\left(L_{\infty}\right) d L_{\infty}+\int_{L_{\infty}^{1}}^{L_{\infty}^{2}} \boldsymbol{\varphi}\left(L_{\infty}\right) \boldsymbol{R}_{\boldsymbol{A}}\left(L_{\infty}\right) d L_{\infty}+\cdots+ \\
& \int_{L_{\infty}^{n-1}}^{L_{\infty}^{n}} \boldsymbol{\varphi}\left(L_{\infty}\right) \boldsymbol{R}_{\boldsymbol{A}}\left(L_{\infty}\right) d L_{\infty}+\int_{L_{\infty}^{n}}^{L_{\infty}^{\max }} \boldsymbol{\varphi}\left(L_{\infty}\right) \boldsymbol{R}_{\boldsymbol{A}}\left(L_{\infty}\right) d L_{\infty}
\end{aligned}
$$

where each integral corresponds to the resulting signal within a narrow bin of LET range. The number of bins represented by $n$ is only limited by the number of uniquely energy dependent detectors.

If we assume small enough bins such that the scintillator response over the bin is relatively flat, the integration shown in equation 6.4 can be rewritten as the sum product of fluence and average response over the LET range. As such, if the boundaries are chosen such that the previous approximation holds, integration could be approximated by a simple multiplication of fluence and response:

$$
M_{i}=\varphi\left(L_{\infty}^{\min t o n}\right) R_{A}\left(L_{\infty}^{\min t o n}\right)+\ldots+\varphi\left(L_{\infty}^{n \text { to } \max }\right) R_{A}\left(L_{\infty}^{n \text { to } \max }\right) .
$$

Written in matrix form, this becomes, 


$$
\left[\begin{array}{c}
M_{1} \\
\vdots \\
M_{n}
\end{array}\right]=\left[\begin{array}{ccc}
R_{i}\left(L_{\infty}^{1}\right) & & R_{i}\left(L_{\infty}^{n}\right) \\
\vdots & \ddots & \vdots \\
R_{n}\left(L_{\infty}^{1}\right) & & R_{n}\left(L_{\infty}^{n}\right)
\end{array}\right]\left[\begin{array}{c}
\varphi\left(L_{\infty}^{i}\right) \\
\vdots \\
\varphi\left(L_{\infty}^{n}\right)
\end{array}\right]
$$

Rearranging for the electron LET fluence vector and assuming the response matrix is non-singular and thus invertible,

$$
\left[\begin{array}{c}
\varphi\left(L_{\infty}^{i}\right) \\
\vdots \\
\varphi\left(L_{\infty}^{n}\right)
\end{array}\right]=\left[\begin{array}{ccc}
R_{i}\left(L_{\infty}^{i}\right) & & R_{i}\left(L_{\infty}^{n}\right) \\
\vdots & \ddots & \vdots \\
R_{n}\left(L_{\infty}^{i}\right) & & R_{n}\left(L_{\infty}^{n}\right)
\end{array}\right]^{-1}\left[\begin{array}{c}
M_{i} \\
\vdots \\
M_{n}
\end{array}\right]
$$

is obtained. Writing in shorthand form,

$$
\boldsymbol{\varphi}\left(\mathbf{L}_{\infty}\right)=\mathbf{R}^{-1} \mathbf{M}
$$

where $\boldsymbol{\varphi}\left(\mathbf{L}_{\infty}\right)$ is the electron LET fluence, $\mathbf{R}^{\mathbf{- 1}}$ is the response matrix, and $\mathbf{M}$ is the measured signal.

If the detectors used have unique energy responses, the response matrix will be invertible, and Equation 6.8 can be used in conjunction with the measured signals from each of the four detectors to obtain the electron fluence in each LET bin. For Equation 6.8 to be valid, the invertibility of the response matrix must be ensured - this is done through the use of differently doped detectors that have energy responses which are sufficiently different in the LET range of interest.

Given the general formalism for LET measurement, proof of principle is shown using four differently lead doped plastic scintillators. These were used to construct a prototype detector, and included the following variants: $0 \%, 1 \%, 1.5 \%$, and $5 \%$ lead doped plastic scintillators. 
In order to increase sensitivity to the low electron energy region (i.e., high LET region of the spectra), exponentially increasing bin sizes in the energy range were used. For the four scintillators $(A-D)$ and bins $(1-4)$ used in this work, Equation 6.7 becomes

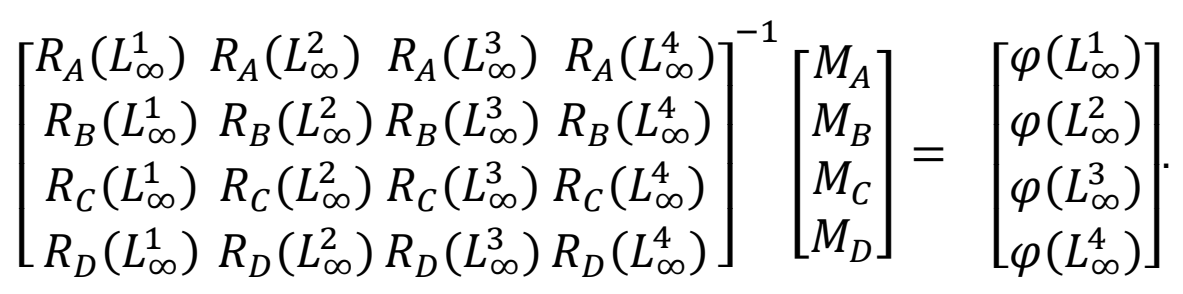

In the following sections, each component of Equation 6.8 will be explained in detail. Section 6.2.2 describes the plastic scintillator measurements and the radiation beams used, and Section 6.2.3 describes the response matrix and how it was obtained. The results obtained using this technique were compared to the LET spectra generated using Monte Carlo Geant4; this will be described in Section 6.2.4.

\subsubsection{Measurements (M)}

In order to determine $\mathbf{M}$ in Equation 6.9, measurements were conducted with four differently doped scintillators in five different radiotherapy photon beams. The scintillators used were differently doped variants of the EJ256 produced by Eljen Technologies (Sweetwater, TX). Lead doping concentrations included the $0 \%, 1.0 \%$, $1.5 \%$, and $5.0 \%$ scintillators. An in-house detector set-up was built which allowed for scintillator measurements in virtual water at a depth of $1.5 \mathrm{~cm}$ from the surface (Figure 6.1A). The setup was designed such that the detector housing was composed of polyethyl ether ketone (PEEK) to achieve water equivalence. Also, it was designed and 
constructed to be light-tight, preventing ambient light around the detector from contributing to noise (Figure 6.1B). A $400 \mu \mathrm{m}$ multimode optical fiber purchased from Thorlabs (Newton, NJ) was used to guide the light produced by each scintillator to a photosensor module. The photosensor used was the Hamamatsu H10721-20 (Shizoka, Japan) due to its superior performance in plastic scintillator measurement relative to other detectors. ${ }^{178} \mathrm{~A}$ photodetector gain of $5 \times 10^{4}$ was used for all measurements.

The beams tested included four different orthovoltage energies (100 kVp, 180 $\mathrm{kVp}, 250 \mathrm{kVp}$, and $300 \mathrm{kVp}$ ) and a Nucletron HDR ${ }^{192} \mathrm{Ir}$ brachytherapy source. These relatively low energy beams were chosen because the electrons produced are of low to medium LET. The orthovoltage beam (Gulmay D3000; Surrey, UK) measurements were done using a $10 \mathrm{~cm}$ open diameter cone with a $30 \mathrm{~cm}$ source to surface distance (Figure 6.2A). A $20 \times 20 \times 20 \mathrm{~cm}$ solid water slab was placed under the beam, and measurements were conducted such that each irradiation delivered 250 Monitor Units.

Brachytherapy measurements were conducted using an identical set-up (Figure 6.2B). The solid water slab containing the scintillator was placed on top of $12 \mathrm{~cm}$ of virtual water. During measurements, 2 Gy of radiation dose was delivered at the scintillator's position using an ${ }^{192}$ Ir source with an air kerma strength of 28721 (cGy $\mathrm{cm}^{2}$ )/hour. This source is of high activity and is thus used for high-dose rate (HDR) brachytherapy. For each beam, measurements were repeated for five times along with a non-scintillator measurement that was conducted to quantify and correct for the Cherenkov light produced. 
For the signal measurement matrix, $\mathbf{M}$, used in Equation 6.9, the scintillator light was normalized to the absorbed dose-to-water delivered over the volume of measurement.

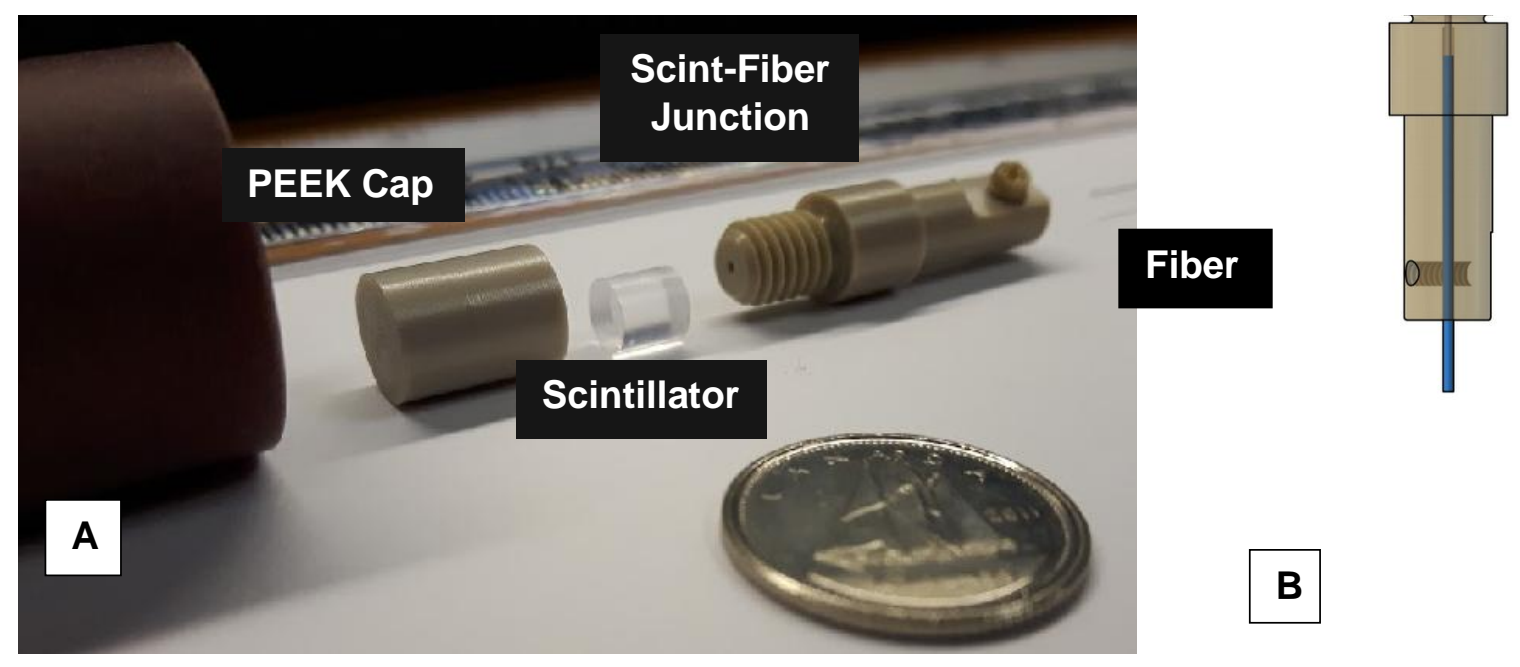

Figure 6.1: (A) Scintillator measurement setup - for each measurement ${ }^{177}$, the scintillators were housed in a detector covering made of polyethyl ether ketone (PEEK) to achieve water equivalence. This unit (shown in beige) is then housed in a virtual water cap (shown in brown, far left) which inserts into the clinical QA virtual water slabs. Canadian dime shown for scale. (B) CAD Design for the detector head shown in the assembled configuration. The optical fiber (shown in blue) rests against the scintillator face; the outer virtual water surface of detector has a length of length is $6 \mathrm{~cm}$, and a diameter of $2.2 \mathrm{~cm}$. 

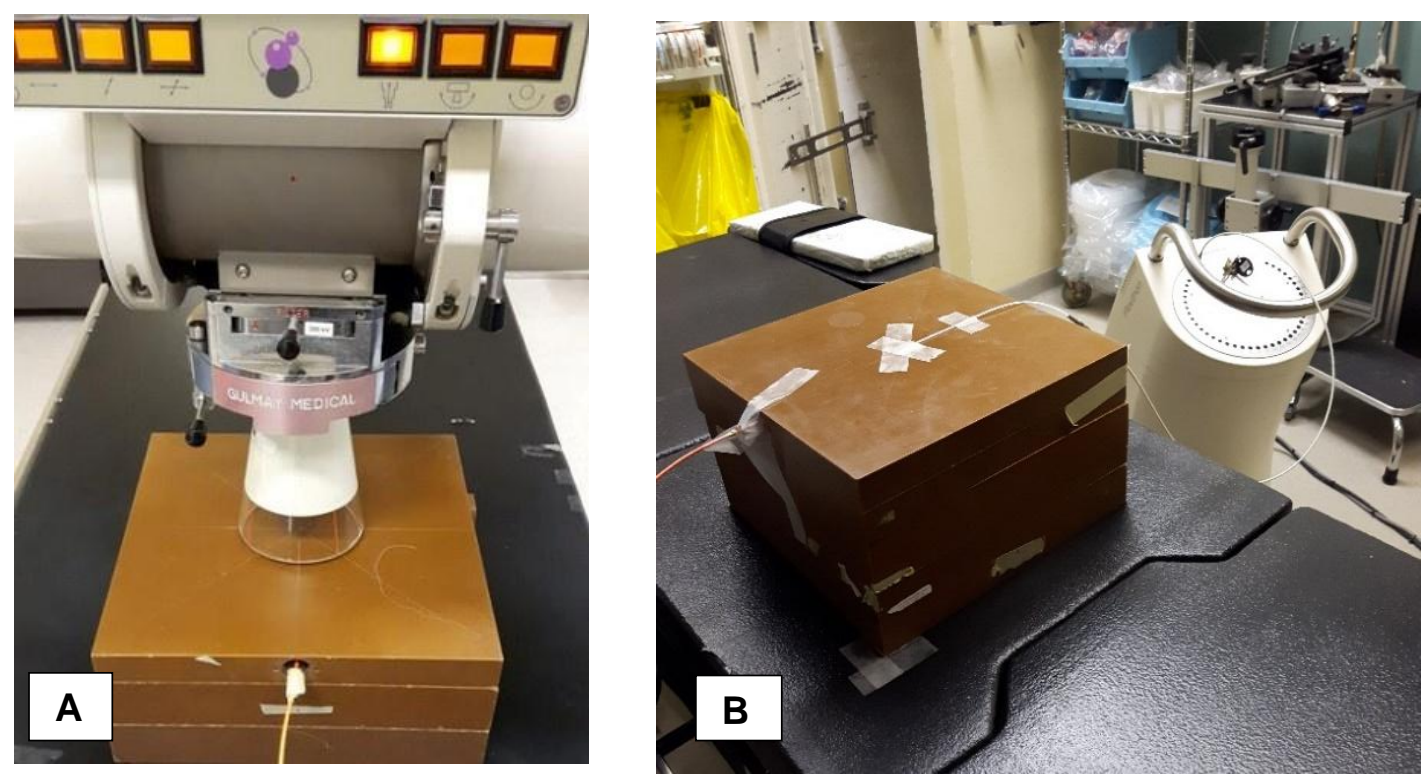

Figure 6.2: (A) Scintillator measurements under 4 different orthovoltage beams. Measurements were conducted using 100, 180, 250, and $300 \mathrm{kVp} \mathrm{x-}$ ray beams using a $30 \mathrm{~cm}$ source to surface distance open cone with a 10 cm diameter. (B) Scintillator measurements using the HDR ${ }^{192}$ Ir source. The detector was placed at a depth of $1.5 \mathrm{~cm}$ from the surface of the virtual water slab, and the HDR source was placed at the surface.

\subsubsection{Response Matrix (R)}

Scintillator response is defined as the value which yields the measured scintillator signal when multiplied with the electron LET fluence. In reality, this quantity must take into account the light emitted by the scintillator, light lost in the optical fiber and at all junctions in the detector setup, photodetector response, and x-ray attenuation. The light lost at the junctions and along the optical fiber, as well as the photodetector response are constant for each of the four scintillators used since their visible light 
emission spectrum remains constant. ${ }^{49}$ These two factors were quantified and found to be insignificant compared to the two major factors which dictate response: scintillator light, and x-ray attenuation. This breakdown of 'response' can be seen in Equation 6.10,

$$
R_{i}\left(L_{\infty}^{i}\right)=\mu_{L}\left(E_{\gamma}\right) \times \int_{L_{\infty}^{i}}^{L_{\infty}^{n}} \frac{\mathrm{d} L}{\mathrm{~d} x} \mathrm{~d} L_{\infty} .
$$

It is important to note that in Equation $6.10, \mu_{L}\left(E_{\gamma}\right)$ refers to the linear attenuation coefficient, $d L / d x$ refers to the visible light emitted by the scintillator, and $L_{\infty}$ refers to the unrestricted LET. As previously mentioned, binning was done according to exponentially increasing electron energies. This allowed for increased sensitivity to the low energy, high LET region of the electron spectra. The energy bin boundaries were of 0 to 0.02 $\mathrm{MeV}, 0.02$ to $0.04 \mathrm{MeV}, 0.04$ to $0.09 \mathrm{MeV}$, and $0.09 \mathrm{MeV}$ onwards.

The light generated by each scintillator used was determined through Birks' Law (Equation 6.1). When using lead doped scintillators, the modified Birks' Law determined by Nusrat et al, ${ }^{177}$ should be used to account for the Z-dependence of $k B$ and $\mathrm{L}_{0}$. As seen in Equation 6.9, $\mathbf{R}$ is a $4 \times 4$ matrix, consisting of rows that correspond to the four differently doped plastic scintillators, and columns that correspond to the four LET bins. For the light calculation, the $k B$ and $\mathrm{L}_{0}$ values were obtained from Nusrat et $\mathrm{al}^{177}$, and the $d E / d x$ values were obtained from the NIST ESTAR ${ }^{157}$ database. Within each LET bin, the visible light generated according to Birks' Law was summated. This was done for each of the four differently doped scintillators used in this work $(0 \%, 1 \%, 1.5 \%, 5 \%$ $\mathrm{Pb})$. 
The linear attenuation coefficients were obtained from the scintillator manufacturer. ${ }^{49}$ Due to lead doping, the attenuation of photons passing through the scintillator volume changes significantly. Acting as a large, thick wall cavity, the large variation in the photon spectrum to differential attenuation inside the scintillators had to be accounted for. In order to improve accuracy, the attenuation coefficients were also obtained from the NIST XCOM database. ${ }^{179}$

\subsubsection{Monte Carlo Simulation}

In order to examine the validity of the initial LET measurement results obtained with the prototype, Monte Carlo simulations were conducted to obtain the true electron LET spectra for each beam. This was done using Geant4.10.3. ${ }^{108}$ To model the orthovoltage beams accurately, SpekCalc® software was used. The resulting x-ray spectra for each orthovoltage beam were validated by comparing Monte Carlo generated depth dose curves to the measured machine depth dose data. For the brachytherapy simulation, the x-ray spectra produced by an ${ }^{192}$ Ir source was obtained from the CLRP Database. ${ }^{107}$ In both simulations, electron energy fluence in water at the position of the scintillator was scored using a $5 \mathrm{~mm}^{3}$ scoring box. Each simulation was run for a total of $10^{9}$ particle histories; the starting seed was changed and the simulation was repeated to obtain the uncertainty of absorbed dose. All simulated electron energy fluences were converted to unrestricted LET fluence using the NIST ESTAR database. ${ }^{157}$ This technique for determining LET has previously been described by Granville et al. ${ }^{158}$ Simulation parameters can be found in Table 6.2, as per the report by AAPM TG268. ${ }^{109}$ 
Table 6.2: Summary of Monte Carlo parameters used. This table is based on AAPM TG268 Report and 'TG268 Item \#' refers to the required Monte Carlo item specified in Sechopolous et al. ${ }^{109}$

\begin{tabular}{|c|c|c|c|}
\hline $\begin{array}{l}\text { TG268 } \\
\text { Item \# }\end{array}$ & Item Name & Description & Ref \\
\hline 2,3 & $\begin{array}{l}\text { Code, version/rel. } \\
\text { date }\end{array}$ & $\begin{array}{l}\text { Geant4 Version } 10.3 \\
\text { Released - Dec. } 92016\end{array}$ & 108 \\
\hline 4,17 & Validation & Refer to Carrier et al., 2004 & 47 \\
\hline 5 & Timing & $\begin{array}{l}\text { Simulation time: } 6 \mathrm{~h} \\
\text { CPU: Intel Xenon; } 20 \text { Cores }\end{array}$ & \\
\hline 8 & $\begin{array}{l}\text { Source } \\
\text { description }\end{array}$ & $\begin{array}{l}\text { - } 100,180,250 \text {, and } 300 \mathrm{kVp} \text { x-rays produced via } \\
\text { SpekCalc } \\
\text { - } \quad{ }^{192} \text { Ir x-ray spectra obtained from CLRP database }\end{array}$ & $\begin{array}{l}14 \\
107\end{array}$ \\
\hline 9 & Cross-sections & $\begin{array}{l}\text { - Physics Package: EMStandardPhysics_Option4 } \\
\text { - PE: G4LivermorePhotoElectricModel } \\
\text { - Compton: G4LivermoreComptonModel (0-2MeV), } \\
\text { G4KleinNishinaModel (2 MeV) } \\
\text { - GK: G4PenelopeGammaConversionModel } \\
\text { - Rayleigh: G4RayleighScattering }\end{array}$ & 110 \\
\hline 10 & $\begin{array}{l}\text { Transport } \\
\text { parameters }\end{array}$ & $\begin{array}{l}\text { - Physics Package: EMStandardPhysics_Option4 } \\
\text { - Multiple Scattering: G4UrbanMscModel95 } \\
\text { - Ionization: G4PenelopelonisationModel } \\
\text { - Brem: G4SeltzerBergerModel } \\
\text { - G4Cutoff: } 10 \mathrm{~nm} \mathrm{e-,} \mathrm{e}^{+}, \mathrm{Y}\end{array}$ & 110 \\
\hline 11 & VRT and/or AEIT & $\mathrm{N} / \mathrm{a}$ & \\
\hline 12 & Scored quantities & $\begin{array}{l}\text { Electron energy fluence using command based } \\
\text { Geant4 scoring }\end{array}$ & \\
\hline
\end{tabular}




\begin{tabular}{|c|c|c|}
\hline 13,18 & $\begin{array}{l}\# \text { of histories/ } \\
\text { statistical } \\
\text { uncertainty }\end{array}$ & $\begin{array}{l}\text { - } 2.2 \times 10^{9} \text { histories per simulation } \\
\text { - Absorbed dose uncertainty }<0.1 \% 1 \mathrm{~cm} \text { away } \\
\text { from source }\end{array}$ \\
\hline 14 & $\begin{array}{l}\text { Statistical } \\
\text { methods }\end{array}$ & $\begin{array}{l}\text { - Absorbed dose uncertainty calculated as root } \\
\text { mean square of energy deposited normalized to } \\
\text { mass }\end{array}$ \\
\hline 15,16 & Post-processing & $\begin{array}{l}\text { Conversion of electron energy fluence to unrestricted } \\
\text { LET using NIST ESTAR database }\end{array}$ \\
\hline
\end{tabular}

\subsection{Results}

In this work, the electron LET spectra at a depth of $1.5 \mathrm{~cm}$ in water were resolved using four differently doped plastic scintillators. In Figure 6.3, the theoretical light produced by each of the four scintillator variants is shown as a function of LET, as explained in Section 6.2.3. Figure 6.4 shows the photon attenuation data for the four differently doped scintillators as obtained from the NIST XCOM database. ${ }^{179}$ In Figure 6.5, each beam's LET fluence spectrum, as determined through Monte Carlo simulation is shown; this was used to evaluate our results based on the technique presented. Figure 6.6 presents the spectra measured for each beam using our proposed methodology and prototype against Monte Carlo. Table 6.3 presents the comparison of the average LETs for each beam. 


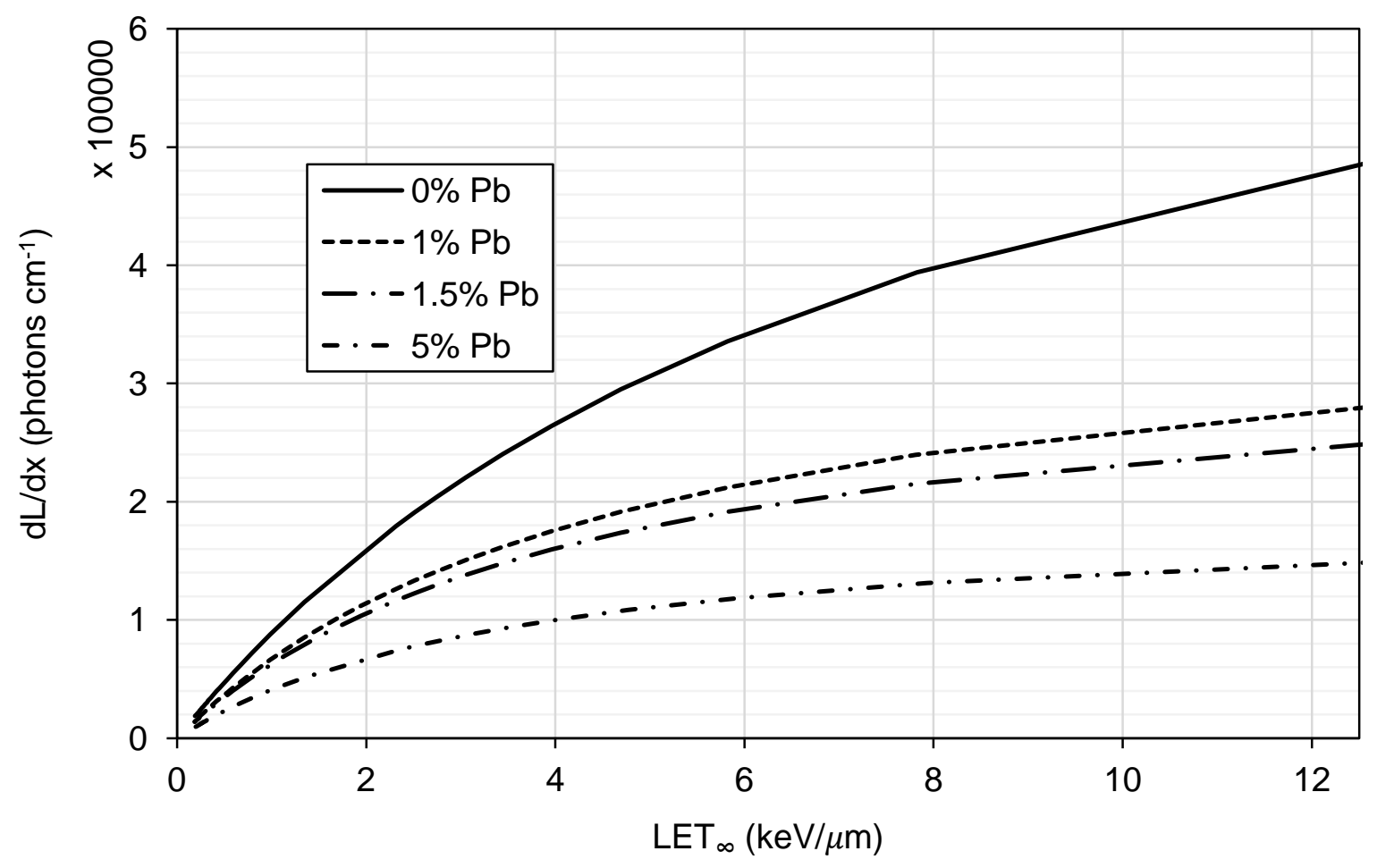

Figure 6.3: Integrated Birks' Law shown for increasing LET in water. Collisional stopping power values were obtained using the NIST ESTAR database, and the appropriate scintillator dependent parameters were chosen to calculate the predicted light output, $\mathrm{dL} / \mathrm{dx}$. 


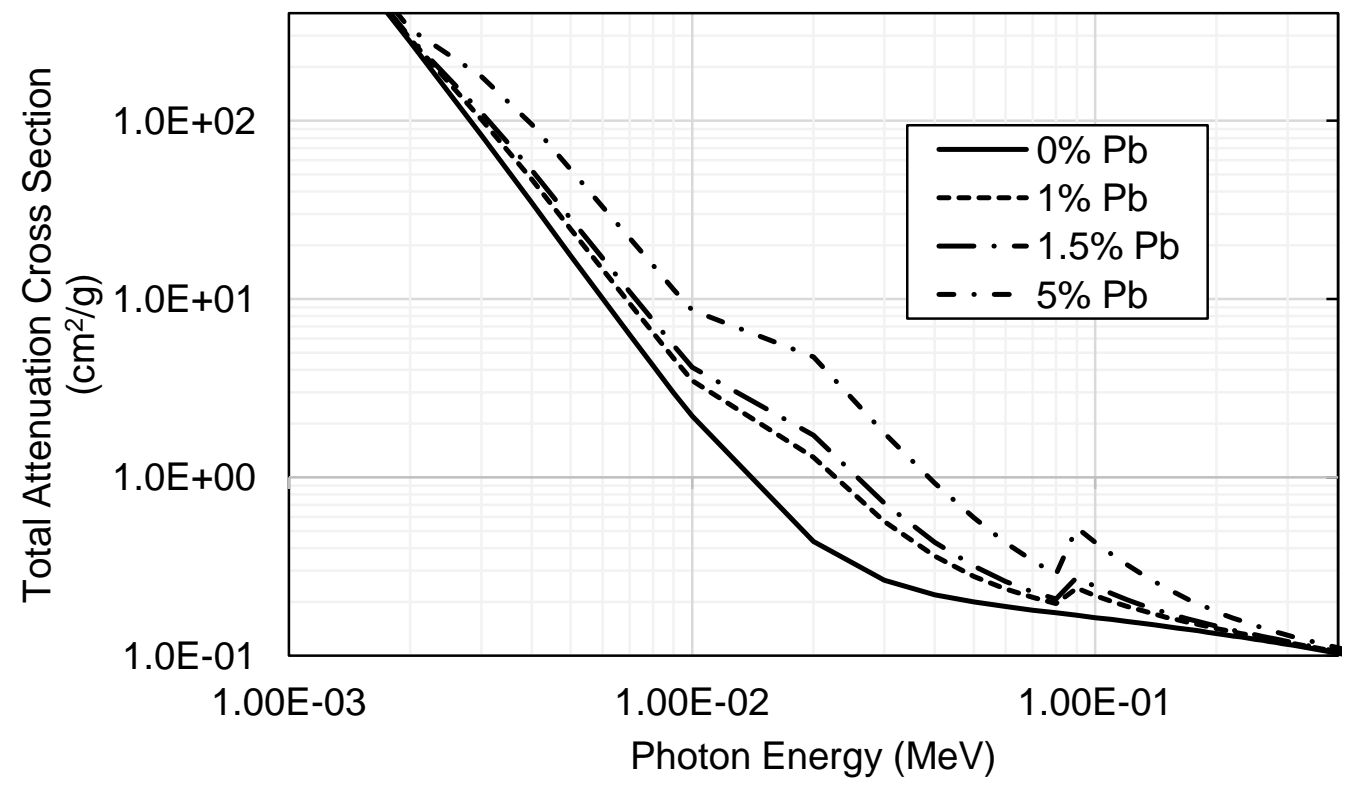

Figure 6.4: Total photon attenuation cross sections for each of the four scintillators used obtained from the NIST XCOM Database ${ }^{179}$

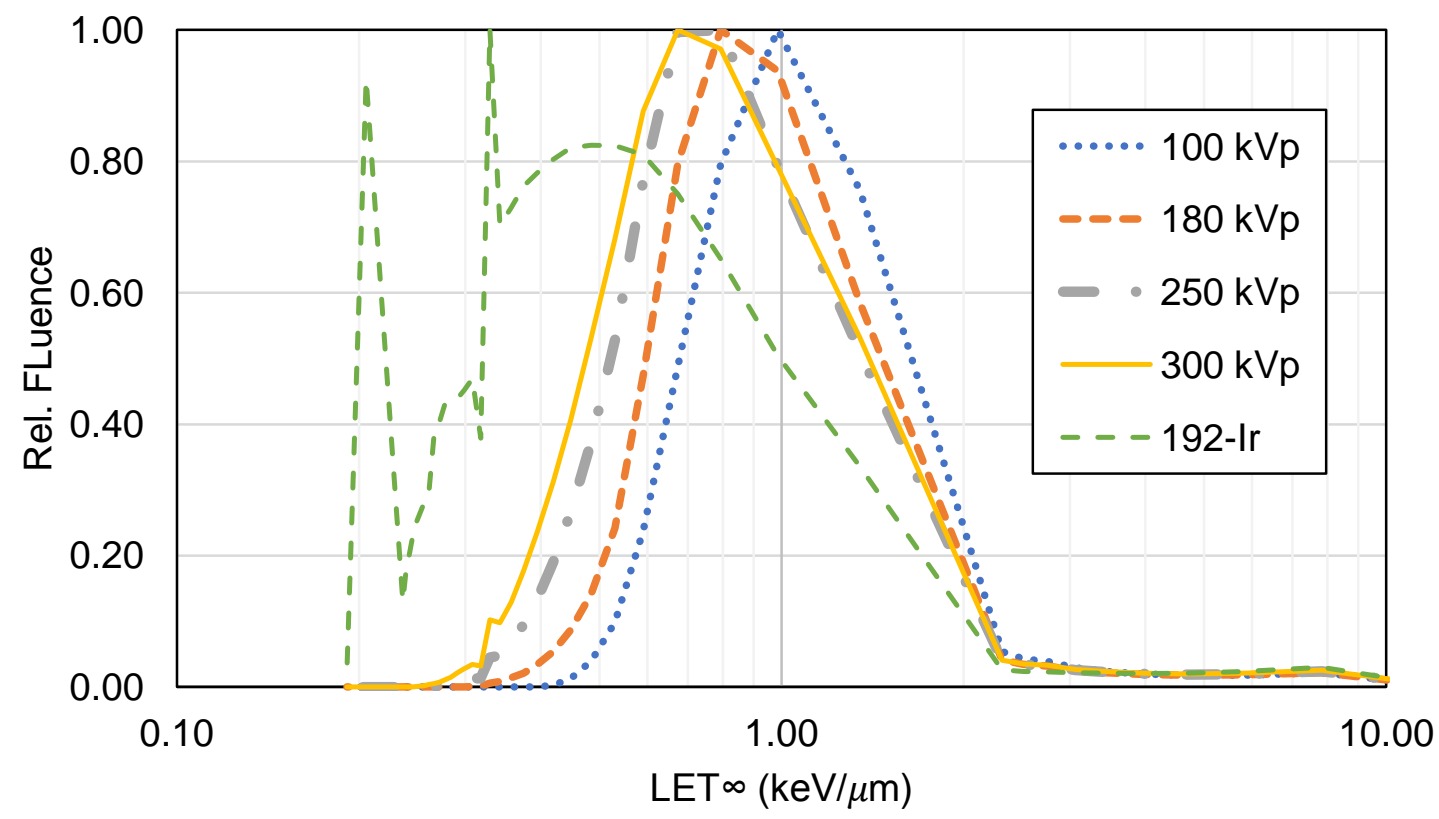

Figure 6.5: Electron LET spectra for each beam at $1.5 \mathrm{~cm}$ depth generated using Monte Carlo GEANT4. 

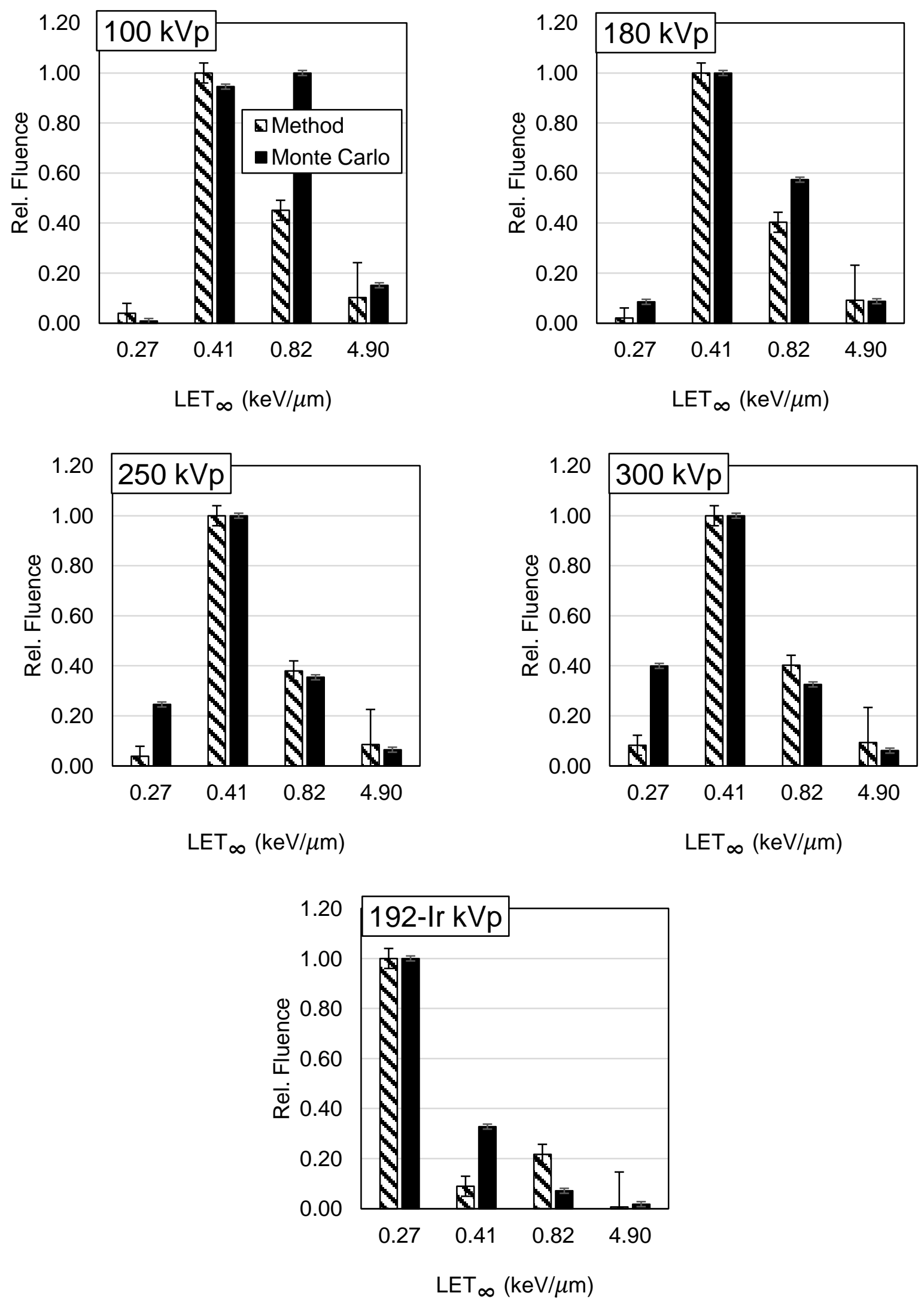
Figure 6.6: Electron LET spectra determined using the method described (diagonal) compared to the Monte Carlo predicted spectra (solid) shown for the $5 \mathrm{x}$-ray beams examined. The error bars shown with the 'method' data correspond to the total uncertainty calculated using the uncertainty analysis shown in Table 6.4. The error bars shown for the Monte Carlo data correspond to the absorbed dose uncertainty after changing the starting simulation seed multiple times $(n=5)$.

Table 6.3: Average LET values calculated using the method described compared to the average LET generated using the Monte Carlo data at a depth of $1.5 \mathrm{~cm}$ in water.

\begin{tabular}{|ccc|}
\hline Beam & $\overline{L_{E} T_{\text {Meas }}}(\mathrm{keV} / \mu \mathrm{m})$ & $\begin{array}{c}\overline{\boldsymbol{L E T}_{\text {Geant }}} \\
(\mathrm{keV} / \mu \mathrm{m})\end{array}$ \\
\hline $100 \mathrm{kVp}$ & 1.18 & 1.72 \\
\hline $180 \mathrm{kVp}$ & 1.10 & 1.20 \\
\hline $250 \mathrm{kVp}$ & 1.17 & 0.99 \\
\hline $300 \mathrm{kVp}$ & 1.12 & 1.00 \\
\hline${ }^{192} \mathrm{Ir}$ & 0.47 & 0.53 \\
\hline
\end{tabular}


Table 6.4: Uncertainty Analysis for measurement and response matrices. All uncertainty components given as relative standard deviations $(k=1)$.

\begin{tabular}{|c|c|c|}
\hline & $\begin{array}{l}\text { 192-Ir } \\
\text { Source (\%) }\end{array}$ & $\begin{array}{l}\text { Orthovoltage } \\
\text { (\%) }\end{array}$ \\
\hline \multicolumn{3}{|l|}{ Measurement Uncertainty } \\
\hline $\begin{array}{l}\text { Reproducibility of } \quad \text { Scintillator } \\
\text { Measurement }(n=5)\end{array}$ & 0.30 & 0.45 \\
\hline Cherenkov effect & 0.54 & 0.28 \\
\hline Manufacturer variation & 0.71 & 0.56 \\
\hline \multicolumn{3}{|l|}{ Positioning Setup } \\
\hline SSD variation & 0 & 1.3 \\
\hline Lateral variation & 0.3 & 0.3 \\
\hline In-phantom depth & 0 & 0 \\
\hline \multicolumn{3}{|l|}{ Response Matrix Uncertainty } \\
\hline \multicolumn{3}{|l|}{ NIST ESTAR $^{157}$} \\
\hline$E_{e-}>100 \mathrm{keV}$ & 2 & 2 \\
\hline $100 \mathrm{keV}>\mathrm{E}_{\mathrm{e}-}>10 \mathrm{keV}$ (high Z) & 7 & 7 \\
\hline \multicolumn{3}{|l|}{ NIST XCOM ${ }^{179}$} \\
\hline $\mathrm{E}_{\gamma}>200 \mathrm{keV}$ & 3 & 3 \\
\hline $1 \mathrm{keV}>\mathrm{E}_{\gamma}>0.5 \mathrm{keV}$ & 12 & 12 \\
\hline Optical fiber light loss & 0.1 & 0.1 \\
\hline Birks' Parameter & 1 & 1 \\
\hline Scintillator - fiber junction & 0.5 & 0.5 \\
\hline \multicolumn{3}{|l|}{ Combined Uncertainty $(k=1)$} \\
\hline High LET & 14 & 14 \\
\hline Low LET & 3.9 & 4.1 \\
\hline
\end{tabular}




\subsection{Discussion}

In this work, a novel technique for measuring the average LET of an x-ray beam was presented. Using differently doped plastic scintillators, a detector comprised of differently energy dependent components was constructed. Five different radiation therapy beams were examined: $100 \mathrm{kVp}, 180 \mathrm{kVp}, 250 \mathrm{kVp}, 300 \mathrm{kVp}$ x-rays, and an ${ }^{192}$ Ir source. For each beam, measurements were conducted using the prototype detector (Figure 6.1) such that the measurement vector, $\mathbf{M}$, was obtained. Next, a response matrix was obtained by integrating the predicted light output of each scintillator using Birks' Law over specific the LET bins. Once the measurement vector and the response matrix were obtained, the energy fluence of radiation traversing the scintillator was determined.

As discussed in section 6.2.3, Birks' Law (Equation 6.1) and the manufacturer provided linear attenuation data were required to obtain $\mathbf{R}$. For increased accuracy, total attenuation coefficients were obtained from NIST XCOM ${ }^{179}$ (Figure 6.4), however, there was no difference in the measured LET spectra - thus, the manufacturer provided linear attenuation data was used. The predicted visible photons produced by each scintillator was shown in Figure 6.3. As can be seen, all four scintillators exhibit a saturation as higher electron LET levels are reached.

Several studies have described the poor performance of plastic scintillators under high LET radiation. ${ }^{132,135,142,154,180-182}$ This is referred to as scintillator quenching. As lead dopant concentration increased, less light was emitted due to the reduction in 
scintillator light yield. ${ }^{177}$ The total attenuation coefficients for photons in the energy ranges relevant to this work are shown in Figure 6.4.

In Figure 6.5, the LET spectra for each beam obtained using Monte Carlo GEANT4 is shown. In the simulation, the electron energy fluence spectra at a depth of $1.5 \mathrm{~cm}$ in water was scored using command-based scoring. These energy spectra were then converted to LET spectra using the technique described by Granville et al. ${ }^{158}$ In this technique, the electron energy spectrum is converted into an LET spectrum using the relation between energy and collisional stopping power found in the NIST ESTAR database. ${ }^{157}$ As expected, the higher energy x-ray beams exhibited a lower LET. The electron energy spectrum produced by the $100 \mathrm{kVp}$ beam was the highest in LET, followed by the $180 \mathrm{kVp}, 250 \mathrm{kVp}, 300 \mathrm{kVp}$, and ${ }^{192} \mathrm{Ir}$ beams. It is also worthy to note the non-symmetric shape of the ${ }^{192}$ Ir electron spectrum in comparison to the relatively symmetrical orthovoltage beam spectra. This is caused by its complex photon emission spectrum. ${ }^{107}$

The results of the proposed LET determination method are shown in Figure 6.6. The measured spectra follow the true electron spectra trend for each beam with the exception of the poor performance in the $100 \mathrm{kVp}$ beam. The mean LETs calculated using both techniques show agreement for all but the $100 \mathrm{kVp}$ beam, as seen in Table 6.3. This is especially significant given the limited difference between the range of LETs examined in this work, as can be seen in Figure 6.3.

As seen in Table 6.4, the uncertainties associated with our measurement can be significant. The uncertainty in the measured electron LET fluence stems from the two components: measurement and response. From the measurement data, the 
uncertainties are mainly caused by beam positioning and setup. In the response matrix, the main sources of uncertainty are the electron stopping power data obtained from the ESTAR database, and the total attenuation cross section data obtained from the NIST XCOM database. For both of these datasets, uncertainties, especially at lower energies and higher $Z$ values can be significant, ranging up to $50 \%$ in some cases. For this work, the high- $\mathrm{Z}(\mathrm{Pb})$ component only made up a maximum of $5 \%$ of the scintillator mass. The Monte Carlo uncertainty in Figure 6.6 corresponds to the uncertainty on absorbed dose in the scintillator volume after changing the starting seed multiple times $(n=5)$.

The main weakness in this technique stem from the low resolution caused by the limited number of differently doped scintillators. Due to only four differently doped plastic scintillators being available, large amounts of data was lost due to averaging. As an example, Figure 6.4 shows the total attenuation photon cross sections as obtained from the NIST XCOM database; there are several small peaks and edges present in the attenuation data due to the lead atom's characteristic x-rays. These effects manifest in the measured signals $(\mathbf{M})$, however, they are lost in the calculation of the response matrix $(\mathbf{R})$ because of the small number of bins. Also, this calculation method assumes that the response and attenuation do not change significantly within the bin width defined.

Despite these weaknesses and uncertainties, a novel way to easily and immediately measure the electron LET spectrum at a given point using differently doped plastic scintillators has been presented. This is the first work of its kind and can lead to potential applications not only in radiation oncology, but in space and health physics dosimetry also. 


\subsection{Conclusions}

This work demonstrates a novel technique for LET measurement. Using differently doped scintillators, the mean LET of five photon beams was determined by calculating the predicted response using Birks' law and photon attenuation data. This work presents a potentially viable energy and LET spectrometry technique; especially once more differently doped scintillators can be easily manufactured and incorporated into the design. Future studies include examining different lead concentrations along with different elemental dopants to provide increased resolution and therefore achieve better agreement with Monte Carlo generated data.

\subsection{Acknowledgements}

The authors would like to acknowledge Harry Easton for their efforts in the fabrication. This work has been supported in part by Discovery grants of the Natural Sciences and Engineering Research Council (NSERC; Grant No. RGPIN-435608 and RGPIN 31178013). 


\section{Conclusions and Future Work}

\subsection{Summary of Findings}

In this section, the results found in the two main parts of the dissertation will be presented and summarized.

\subsubsection{Biological Impact of Increased Scattered Radiation in a 6 MV x-ray Beam}

In this chapter (Chapter 3), the maximum RBE was calculated along a $6 \mathrm{MV}$ photon beam's profile. The linear accelerator examined was the Varian Clinac 600C. Using a phase-space file, radiation exiting the head of the linear accelerator was set incident upon a water tank using Geant4; the resulting electron fluences at 1.5 and $5 \mathrm{~cm}$ deep were scored using logarithmically increasing energy bins. Next, the electron fluence spectrum at each voxel was used to calculate the $\alpha / \beta$ ratio as per the Zaider

and Rossi. ${ }^{67}$ The results from this chapter reveal several interesting points. As shown in Figure 3.4, the out-of-field RBE increase is more pronounced for the more collimated beam $\left(1 \times 1 \mathrm{~cm}^{2}\right)$. The fact that smaller beamlets exhibit increased out-of-field RBE variations is significant in the context of IMRT. It is generally accepted that for 3D 
conformal treatments, the out-of-field changes are clinically insignificant. This can be seen by simply examining the electron fluence for the $10 \times 10 \mathrm{~cm}^{2}$ open field beam in Figure 3.5. However, for highly modulated treatments, the surrounding region contains increased scattered photons and electrons which may result in an increased effective dose to organs at risk. ${ }^{62,64,171}$

\subsubsection{Maximum RBE Variation in Brachytherapy}

In Chapter 4, the same methodology was used to examine radiobiological variation surrounding three brachytherapy sources: ${ }^{192} \mathrm{Ir},{ }^{125} \mathrm{I}$, and ${ }^{169} \mathrm{Yb}$. The emission spectra (post-casing) for each of the three sources was obtained from the CLRP brachytherapy source database ${ }^{107}$, and simulations were conducted in water using Geant4. The electron fluence at the position of each respective source was compared to the fluence at various distances away. The initial validation of lodine-125 relative to Iridium-192 revealed that the technique did indeed work and was consistent with previously reported data. One of the most interesting results from this chapter was the large maximum RBE increase observed in Ytterbium-169 at the periphery. This source is not currently available on the market, however, a potential RBE-enhancement effect along with its relatively long half life and low toxicity could cause interest in clinical applications to grow. The $3.6 \% \mathrm{RBE}_{\mathrm{M}}$ hotspot observed at the periphery of the Iridium192 high dose rate prostate plan was interesting, but most likely clinically insignificant. At $8 \mathrm{~cm}$ away, the radiation fluence and resulting absorbed dose are so diminished that a $3.6 \%$ boost would be negligible. 


\subsubsection{Lead-doped Plastic Scintillators}

In Chapter 5, the impact of lead doping on plastic scintillator response was quantified. Prior to this, lead doped scintillators were used sparsely for health physics applications, and the dosimetric impact of lead in this context had not yet been examined. The presence of lead makes the plastic scintillators energy dependent, as can be seen from Figure 5.4. The utility of this energy dependence is revealed in the next chapter, where the unique energy dependence allows for the unfolding of the electron LET spectra. High-Z doped plastic scintillators are not widely available on the market; the two main scintillator manufacturers both sell lead doped scintillators at a few select concentrations. Eljen Technologies is reported to be developing a Bismuth-doped plastic scintillator. Once this is available, the universality of Equations 5.2 and 5.3 for non-lead dopants can be examined.

\subsubsection{LET Measurement}

In Chapter 6, the LET fluence spectra at a depth of $1.5 \mathrm{~cm}$ in water was measured. The general formalism for the measurement technique was based upon the principle that the light signal produced by a scintillator was a function of scintillator response $(\mathbf{R})$, and $\boldsymbol{\varphi}$, the electron fluence traversing the scintillator. Given that signal was measured using a photodetector and response was determined using the modified Birks' Law and photon attenuation coefficients, the LET fluence was resolvable. Only four differently doped detectors were available, as such, the resolution was limited to four bins. Despite 
marginal performance in the $100 \mathrm{kVp} x$-ray beam and large uncertainties in the mediumhigh LET region, the detector was able to approximately resolve the spectra for the $180 \mathrm{kVp}, 250 \mathrm{kVp}, 300 \mathrm{kVp}$, and Iridium-192 x-ray beams. The average LET obtained from the spectra match closely to that obtained from Monte Carlo, with the exception of $100 \mathrm{kVp}$ beam. This work was a proof-of-concept for the instrument and the methodology. Once more scintillators are obtained, this technique can have many applications not only in radiation oncology, but in other areas of radiation measurement. LET dosimeters would be very useful for space travel, health physics, and nuclear safety applications along with the numerous applications in medicine.

\subsection{Future Work}

Future work mainly involves: 1) exploring the measurement technique under a proton therapy beam, and 2) increasing the number of uniquely doped scintillator thereby increasing resolution. While out-of-field RBE has been shown to change, the need for LET dosimeters in photon therapy quality assurance is not an immediate need. However, in particle therapy, real-time LET dosimeters would prove very valuable, allowing for more accurate biological-effect weighting during treatment planning. As mentioned previously, resolution is currently limited to four bins due to only four uniquely doped scintillators being available. Our group has since purchased a $2.5 \% \mathrm{~Pb}-$ doped plastic scintillator and are trying to obtain Bismuth-doped plastic scintillators from Eljen Technologies. These additional energy dependent detectors will allow for increased accuracy during measurement. 


\section{References}

1. de Oliveira C, Weir S, Rangrej J, et al. The economic burden of cancer care in Canada: a population-based cost study. C open. 2018;6(1):E1-E10. doi:10.9778/cmajo.20170144

2. Hall EJ, Giaccia AJ. Radiobiology for the Radiologist.

3. Smith L, Bryan S, De P, et al. Canadian Cancer Statistics: A 2018 Special Report on Cancer Incidence by Stage.; 2018.

http://www.cancer.ca/ /media/cancer.ca/CW/cancer information/cancer 101/Canadian cancer statistics/Canadian-Cancer-Statistics-2018-EN.pdf?la=en. Accessed June 10, 2019.

4. American Cancer Society Medical and Editorial Team. Radiation Therapy Basics. https://www.cancer.org/treatment/treatments-and-side-effects/treatmenttypes/radiation/basics.html. Published 2019. Accessed July 3, 2019.

5. Podgoršak EB (Ervin B. Radiation Physics for Medical Physicists. Springer; 2010.

6. Richards AA. X Rays and Gamma Rays: Crookes Tubes and Nuclear Light. In: Alien Vision: Exploring the Electromagnetic Spectrum with Imaging Technology, Second Edition. 1000 20th Street, Bellingham, WA 98227-0010 USA: SPIE; 2011:89-113. doi:10.1117/3.883085.ch4

7. Gaspar M. IAEA Receives Medical Linear Accelerator under Partnership from 
Manufacturer | IAEA. IAEA Office of Public Information and Communication. https://www.iaea.org/newscenter/news/iaea-receives-medical-linear-acceleratorunder-partnership-from-manufacturer. Accessed June 10, 2019.

8. Dobranowski A. Behind the research: Sunnybrook's first human image taken on MR-Linac. Your Health Matters - Sunnybrook.

http://health.sunnybrook.ca/research/first-human-image-mr-linac/. Published 2019. Accessed June 12, 2019.

9. Capala J, Goetsch SJ, Orton CG. Point/Counterpoint. Medical use of all high activity sources should be eliminated for security concerns. Med Phys. 2015;42(12):6773-6775. doi:10.1118/1.4934823

10. Canadian Nuclear Safety Commission (CNSC). Nuclear Safety and Control Act. Government of Canada; 1997. https://laws-lois.justice.gc.ca/eng/acts/N28.3/index.html. Accessed June 11, 2019.

11. Spina A, Boari N, Gagliardi F, Donofrio CA, Franzin A, Mortini P. The current role of Gamma Knife radiosurgery in the management of intracranial haemangiopericytoma. Acta Neurochir (Wien). 2016;158(4):635-642. doi:10.1007/s00701-016-2742-3

12. The Image-Guided GammaKnife Story. Techna Institute. https://technainstitute.com/the-image-guided-gammaknife-story/. Published 2016. Accessed June 11, 2019.

13. Das IJ, Paganetti H. Principles and Practice of Proton Beam Therapy. American Association of Physicists in Medicine. Summer School; 2015. 
14. Poludniowski G, Landry G, DeBlois F, Evans PM, Verhaegen F. SpekCalc : a program to calculate photon spectra from tungsten anode x-ray tubes. Phys Med Biol. 2009;54(19):N433-N438. doi:10.1088/0031-9155/54/19/N01

15. Gao W, Raeside DE. Orthovoltage radiation therapy treatment planning using Monte Carlo simulation: treatment of neuroendocrine carcinoma of the maxillary sinus. Phys Med Biol. 1997;42(12):2421-2433. http://www.ncbi.nlm.nih.gov/pubmed/9434298. Accessed July 3, 2019.

16. Brachytherapy. Fox Chase Cancer Centre. https://www.foxchase.org/clinicalcare/conditions/prostate-cancer/treatment/radiation-therapy/brachytherapy. Published 2019. Accessed June 12, 2019.

17. Saha GB. Physics and Radiobiology of Nuclear Medicine. New York, NY: Springer New York; 2013. doi:10.1007/978-1-4614-4012-3

18. Yang $Y$, Xing L. Towards biologically conformal radiation therapy (BCRT): Selective IMRT dose escalation under the guidance of spatial biology distribution. Med Phys. 2005;32(6):1473-1484. doi:10.1118/1.1924312

19. Ilicic K, Combs SE, Schmid TE. New insights in the relative radiobiological effectiveness of proton irradiation. Radiat Oncol. 2018;13(1):6. doi:10.1186/s13014-018-0954-9

20. Manning MA, Zwicker RD, Arthur DW, Arnfield M. Biologic treatment planning for high-dose-rate brachytherapy. Int J Radiat Oncol. 2001;49(3):839-845. doi:10.1016/S0360-3016(00)01453-X 
21. Britten RA, Nazaryan V, Davis LK, et al. Variations in the RBE for Cell Killing Along the Depth-Dose Profile of a Modulated Proton Therapy Beam. Radiat Res. 2013;179(1):21-28. doi:10.1667/RR2737.1

22. Nusrat H, Pang G, Ahmad SB, Sarfehnia A. Evaluating the biological impact of increased scattered radiation in single and composite field radiation beams. Biomed Phys Eng Express. 2018;4(3):035016. doi:10.1088/2057-1976/aab0db

23. Howell RM, Hertel NE, Wang Z, Hutchinson J. RBE or effective dose to evaluate the effect of different beam energies for intensity modulated radiation therapy. Nucl Instruments Methods Phys Res Sect A Accel Spectrometers, Detect Assoc Equip. 2006;562(2):1024-1028. doi:10.1016/J.NIMA.2006.02.087

24. Dale RG, Jones B, Cárabe-Fernández A. Why more needs to be known about RBE effects in modern radiotherapy. Appl Radiat Isot. 2009;67(3):387-392. doi:10.1016/J.APRADISO.2008.06.013

25. Chaudhary P, Marshall TI, Perozziello FM, et al. Relative biological effectiveness variation along monoenergetic and modulated Bragg peaks of a $62-\mathrm{MeV}$ therapeutic proton beam: a preclinical assessment. Int J Radiat Oncol Biol Phys. 2014;90(1):27-35. doi:10.1016/j.jijobp.2014.05.010

26. Dale R, Carabe-Fernandez A. The Radiobiology of Conventional Radiotherapy and Its Application to Radionuclide Therapy. Cancer Biother Radiopharm. 2005;20(1):47-51. doi:10.1089/cbr.2005.20.47

27. Simon SL, Bailey SM, Beck HL, et al. Estimation of Radiation Doses to U.S. Military Test Participants from Nuclear Testing: A Comparison of Historical Film- 
Badge Measurements, Dose Reconstruction and Retrospective Biodosimetry. Radiat Res. 2019;191(4):297. doi:10.1667/RR15247.1

28. Chancellor JC, Blue RS, Cengel KA, et al. Limitations in predicting the space radiation health risk for exploration astronauts. NPJ microgravity. 2018;4:8. doi:10.1038/s41526-018-0043-2

29. Giantsoudi D, Grassberger C, Craft D, Niemierko A, Trofimov A, Paganetti H. Linear Energy Transfer-Guided Optimization in Intensity Modulated Proton Therapy: Feasibility Study and Clinical Potential. Int J Radiat Oncol. 2013;87(1):216-222. doi:10.1016/J.IJROBP.2013.05.013

30. Kraft G, KrÄMer M. Linear Energy Transfer and Track Structure. Adv Radiat Biol. 1993;17:1-52. doi:10.1016/B978-0-12-035417-7.50004-0

31. Hunter N, Muirhead CR. Review of relative biological effectiveness dependence on linear energy transfer for low-LET radiations. J Radiol Prot. 2009;29(1):5-21. doi:10.1088/0952-4746/29/1/R01

32. Wedenberg M, Toma-Dasu I. Disregarding RBE variation in treatment plan comparison may lead to bias in favor of proton plans. Med Phys. 2014;41(9):091706. doi:10.1118/1.4892930

33. Britten RA, Nazaryan V, Davis LK, et al. Variations in the RBE for Cell Killing Along the Depth-Dose Profile of a Modulated Proton Therapy Beam. Radiat Res. 2013;179(1):21-28. doi:10.1667/RR2737.1

34. Calugaru V, Nauraye C, Noël G, Giocanti N, Favaudon V, Mégnin-Chanet F. 
Radiobiological characterization of two therapeutic proton beams with different initial energy spectra used at the Institut Curie Proton Therapy Center in Orsay. Int J Radiat Oncol Biol Phys. 2011;81(4):1136-1143.

doi:10.1016/j.ijrobp.2010.09.003

35. Wouters BG, Skarsgard LD, Gerweck LE, et al. Radiobiological Intercomparison of the $160 \mathrm{MeV}$ and $230 \mathrm{MeV}$ Proton Therapy Beams at the Harvard Cyclotron Laboratory and at Massachusetts General Hospital. Radiat Res. 2015;183(2):174187. doi:10.1667/RR13795.1

36. Cuaron JJ, Chang C, Lovelock M, et al. Exponential Increase in Relative Biological Effectiveness Along Distal Edge of a Proton Bragg Peak as Measured by Deoxyribonucleic Acid Double-Strand Breaks. Int J Radiat Oncol Biol Phys. 2016;95(1):62-69. doi:10.1016/j.jijrobp.2016.02.018

37. Sørensen BS. Radiobiology. http://phys.au.dk/forskning/forskningsomraader/aptg0/research/radiobiology/. Published 2018. Accessed July 3, 2019.

38. Howell RW, Azure MT, Narra VR, Rao D V. Relative biological effectiveness of alpha-particle emitters in vivo at low doses. Radiat Res. 1994;137(3):352-360. http://www.ncbi.nlm.nih.gov/pubmed/8146279. Accessed June 7, 2019.

39. Willers H, Dahm-Daphi J, Powell SN. Repair of radiation damage to DNA. Br J Cancer. 2004;90(7):1297-1301. doi:10.1038/sj.bjc.6601729

40. Cohen L, Awschalom M. Fast Neutron Radiation Therapy. Annu Rev Biophys Bioeng. 1982;11(1):359-390. doi:10.1146/annurev.bb.11.060182.002043 
41. Andreo P. Monte Carlo simulations in radiotherapy dosimetry. Radiat Oncol. 2018;13(1):121. doi:10.1186/s13014-018-1065-3

42. Vassiliev ON. Monte Carlo Methods for Radiation Transport: Fundamentals and Advanced Topics.

43. Kawrakow I. Accurate condensed history Monte Carlo simulation of electron transport. I. EGSnrc, the new EGS4 version. Med Phys. 2000;27(3):485-498. doi:10.1118/1.598917

44. Baró J, Sempau J, Fernández-Varea JM, Salvat F. PENELOPE: An algorithm for Monte Carlo simulation of the penetration and energy loss of electrons and positrons in matter. Nucl Instruments Methods Phys Res Sect B Beam Interact with Mater Atoms. 1995;100(1):31-46. doi:10.1016/0168-583X(95)00349-5

45. Agostinelli S, Allison J, Amako K, et al. Geant4-a simulation toolkit. Nucl Instruments Methods Phys Res Sect A Accel Spectrometers, Detect Assoc Equip. 2003;506(3):250-303. doi:10.1016/S0168-9002(03)01368-8

46. Ali Nedaie $\mathrm{H}$, Mosleh-Shirazi MA, Gharaati $\mathrm{H}$, Shariari M, Allahverdi M. Assessment of different MCNP Monte Carlo codes in electron absorbed dose. Reports Pract Oncol Radiother. 2006;11(6):293-298. doi:10.1016/S15071367(06)71075-6

47. Carrier J-F, Archambault L, Beaulieu L, Roy R. Validation of GEANT4 , an objectoriented Monte Carlo toolkit, for simulations in medical physics. Med Phys. 2004;31(3):484-492. doi:10.1118/1.1644532 
48. Capote R, Jeraj $\mathrm{R}, \mathrm{Ma} \mathrm{CM}$, et al. Phase-space database for external beam radiotherapy. Summary report of a consultants' meeting. 2006. https://inis.iaea.org/search/search.aspx?orig_q=RN:37073778. Accessed July 6, 2017.

49. Eljen Technology. EJ-256 Eljen Datasheet. Sweetwater, TX; 2016.

50. Standard Imaging. Exradin W2 Scintillator Datasheet. May 2019. https://www.standardimaging.com/exradin/scintillators/. Accessed July 3, 2019.

51. Beddar AS, Mackie TR, Attix FH. Water-equivalent plastic scintillation detectors for high-energy beam dosimetry: I. Physical characteristics and theoretical considerations. Phys Med Biol. 1992;37(10):1883-1900. doi:10.1088/0031$9155 / 37 / 10 / 006$

52. Birks JB. The specific fluorescence of anthracene and other organicmaterials [5]. Phys Rev. 1951;84(2):364-365. doi:10.1103/PhysRev.84.364.2

53. Chaney EL, Cullip TJ, Gabriel TA. A Monte Carlo study of accelerator head scatter. Med Phys. 1994;21(9):1383-1390. doi:10.1118/1.597194

54. Paganetti H. Relative biological effectiveness (RBE) values for proton beam therapy. Variations as a function of biological endpoint, dose, and linear energy transfer. Phys Med Biol. 2014;59(22):R419-R472. doi:10.1088/00319155/59/22/R419

55. Kirkby C, Field C, MacKenzie M, Syme A, Fallone BG. A Monte Carlo study of the variation of electron fluence in water from a $6 \mathrm{MV}$ photon beam outside of the 
field. Phys Med Biol. 2007;52(12):3563-3578. doi:10.1088/0031-9155/52/12/015

56. Kirkby C, Field C, MacKenzie M, Syme A, Fallone BG. A Monte Carlo study of the variation of electron fluence in water from a $6 \mathrm{MV}$ photon beam outside of the field. Phys Med Biol. 2007;52(12):3563-3578. doi:10.1088/0031-9155/52/12/015

57. Liu HH, Verhaegen F. An investigation of energy spectrum and lineal energy variations in mega-voltage photon beams used for radiotherapy. Radiat Prot Dosimetry. 2002;99(1-4):425-427.

http://www.ncbi.nlm.nih.gov/pubmed/12194346. Accessed July 6, 2017.

58. Moiseenko V, Mulligan M, Kron T. Radiation quality of a tomotherapy photon fan beam. Health Phys. 2004;87(2):166-170. doi:10.1097/00004032-20040800000005

59. Butterworth KT, McGarry CK, Trainor C, O'Sullivan JM, Hounsell AR, Prise KM. Out-of-field cell survival following exposure to intensity-modulated radiation fields. Int J Radiat Oncol Biol Phys. 2011;79(5):1516-1522.

doi:10.1016/j.ijrobp.2010.11.034

60. Trainor C, Butterworth KT, McGarry CK, et al. DNA Damage Responses following Exposure to Modulated Radiation Fields. Koritzinsky M, ed. PLoS One. 2012;7(8):e43326. doi:10.1371/journal.pone.0043326

61. Trainor C, Butterworth KT, McGarry CK, et al. Cell survival responses after exposure to modulated radiation fields. Radiat Res. 2012;177(1):44-51. http://www.ncbi.nlm.nih.gov/pubmed/22029841. Accessed November 8, 2017. 
62. McGarry CK, Butterworth KT, Trainor C, et al. In-vitro investigation of out-of-field cell survival following the delivery of conformal, intensity-modulated radiation therapy (IMRT) and volumetric modulated arc therapy (VMAT) plans. Phys Med Biol. 2012;57(20):6635-6645. doi:10.1088/0031-9155/57/20/6635

63. Chofor N, Harder D, Willborn KC, Poppe B. Internal scatter, the unavoidable major component of the peripheral dose in photon-beam radiotherapy. Phys Med Biol. 2012;57(6):1733-1743. doi:10.1088/0031-9155/57/6/1733

64. Ezzati AO, Xiao Y, Sohrabpour M, Studenski MT. The effect of energy spectrum change on DNA damage in and out of field in 10-MV clinical photon beams. Med Biol Eng Comput. 2015;53(1):67-75. doi:10.1007/s11517-014-1213-3

65. Okamoto $\mathrm{H}$, Kanai $\mathrm{T}$, Kase $\mathrm{Y}$, et al. Relation between lineal energy distribution and relative biological effectiveness for photon beams according to the microdosimetric kinetic model. J Radiat Res. 2011;52(1):75-81. http://www.ncbi.nlm.nih.gov/pubmed/21160135. Accessed November 8, 2017.

66. Dale RG, Jones B. The assessment of RBE effects using the concept of biologically effective dose. Int J Radiat Oncol. 1999;43(3):639-645. doi:10.1016/S0360-3016(98)00364-2

67. Zaider M, Rossi HH. On the application of microdosimetry to radiobiology. Radiat Res. 1988;113(1):15-24. http://www.ncbi.nlm.nih.gov/pubmed/3340718. Accessed July 6, 2017.

68. Kellerer AM, Rossi HH. The theory of dual radiation action. Curr Top Radiat Res. 1972;8:85-158. 
69. Chen J, Kellerer AM. Proximity functions for electrons from $100 \mathrm{eV}$ to $10 \mathrm{MeV}$. Radiat Prot Dosimetry. 2007;122(1-4):56-60. doi:10.1093/rpd/ncl412

70. Brualla L, Salvat F, Palanco-Zamora R. Efficient Monte Carlo simulation of multileaf collimators using geometry-related variance-reduction techniques. Phys Med Biol. 2009;54(13):4131-4149. doi:10.1088/0031-9155/54/13/011

71. Bednarz B, Xu XG. Monte Carlo modeling of a 6 and 18 MV Varian Clinac medical accelerator for in-field and out-of-field dose calculations: development and validation. Phys Med Biol. 2009;54(4):N43-N57. doi:10.1088/0031$9155 / 54 / 4 / \mathrm{N} 01$

72. Kry SF, Titt U, Followill D, et al. A Monte Carlo model for out-of-field dose calculation from high-energy photon therapy. Med Phys. 2007;34(9):3489-3499. doi:10.1118/1.2756940

73. Nikjoo H, Lindborg L. RBE of low energy electrons and photons. Phys Med Biol. 2010;55(10). doi:10.1088/0031-9155/55/10/R01

74. Reniers B, Liu D, Rusch T, Verhaegen F. Calculation of relative biological effectiveness of a low-energy electronic brachytherapy source. Phys Med Biol. 2008;53(24):7125-7135. doi:10.1088/0031-9155/53/24/008

75. Malin MJ, Palmer BR, DeWerd LA. Absolute measurement of LDR brachytherapy source emitted power: Instrument design and initial measurements. Med Phys. 2016;43(2):796-806. doi:10.1118/1.4939666

76. Granero D, Pérez-Calatayud J, Casal E, Ballester F, Venselaar J. A dosimetric 
study on the Ir-192 high dose rate Flexisource. Med Phys. 2006;33(12):45784582. doi:10.1118/1.2388154

77. Famulari G, Pater P, Enger SA. Microdosimetric Evaluation of Current and Alternative Brachytherapy Sources-A Geant4-DNA Simulation Study. Int J Radiat Oncol Biol Phys. 2018;100(1):270-277. doi:10.1016/j.jijrobp.2017.09.040

78. Holloway RP, Dale RG. Theoretical implications of incorporating relative biological effectiveness into radiobiological equivalence relationships. Br J Radiol. 2013;86(1022):20120417. doi:10.1259/bjr.20120417

79. Marthinsen ABL, Gisetstad R, Danielsen S, Frengen J, Strickert T, Lundgren S. Relative biological effectiveness of photon energies used in brachytherapy and intraoperative radiotherapy techniques for two breast cancer cell lines. Acta Oncol (Madr). 2010;49(8):1261-1268. doi:10.3109/0284186X.2010.504226

80. White SA, Reniers B, de Jong EEC, Rusch T, Verhaegen F. A comparison of the relative biological effectiveness of low energy electronic brachytherapy sources in breast tissue: a Monte Carlo study. Phys Med Biol. 2016;61(1):383-399. doi:10.1088/0031-9155/61/1/383

81. Bernstein M, Gutin PH, Weaver KA, Deen DF, Barcellos MH. 125 I Interstitial Implants in the RIF-1 Murine Flank Tumor: An Animal Model for Brachytherapy. Radiat Res. 1982;91(3):624. doi:10.2307/3575897

82. Freeman ML, Goldhagen P, Sierra E, Hall EJ. Studies with encapsulated $125 \mathrm{I}$ sources. II. determination of the relative biological effectiveness using cultured mammalian cells. Int J Radiat Oncol. 1982;8(8):1355-1361. doi:10.1016/0360- 
3016(82)90586-7

83. Da Silva VF, Gutin PH, Deen DF, Weaver KA. Relative biological effectiveness of I-125 sources in a murine brachytherapy model. Int J Radiat Oncol Biol Phys. 1984;10:2109-2111.

84. Kwan D, Kagan R, Norman A. Relative biological effectiveness of 1251 in the induction of micronuclei in human peripheral blood lymphocytes. Radiother Oncol. 1985;4:163-166.

85. Hering ER, Sealy GR, Dowman P, Blekkenhorst G. OER and RBE for $125 \mathrm{I}$ and 192Ir at low dose rate on mammalian cells. Radiother Oncol. 1987;10(3):247-252. http://www.ncbi.nlm.nih.gov/pubmed/3432600. Accessed November 13, 2018.

86. Wuu CS, Kliauga P, Zaider M, Amols HI. Microdosimetric evaluation of relative biological effectiveness for Pd-103, I-125, Am-241, and Ir-192 brachytherapy sources. Int J Radiat Oncol Bio Phys. 1996;36(3):689-687.

87. Ling CC, Li WX, Anderson LL. The relative biological effectiveness of I-125 and Pd-103. Int J Radiat Oncol Biol Phys. 1995;32(2):373-378.

88. Rava P, Dvorak T, Markelewicz RJ, et al. A comparison of the biological effective dose of 50-kV electronic brachytherapy with 192Ir high-dose-rate brachytherapy for vaginal cuff irradiation. Brachytherapy. 2012;11(5):402-407. doi:10.1016/j.brachy.2011.08.004

89. Herskind C, Steil V, Kraus-Tiefenbacher U, Wenz F. Radiobiological Aspects of Intraoperative Radiotherapy (IORT) with Isotropic Low-Energy X Rays for Early- 
Stage Breast Cancer. Radiat Res. 2005;163(2):208-215. doi:10.1667/RR3292

90. Dickler A, Kirk MC, Coon A, et al. A dosimetric comparison of Xoft Axxent Electronic Brachytherapy and iridium-192 high-dose-rate brachytherapy in the treatment of endometrial cancer. Brachytherapy. 2008;7(4):351-354. doi:10.1016/j.brachy.2008.05.003

91. Brenner DJ, Leu CS, Beatty JF, Shefer RE. Clinical relative biological effectiveness of low-energy $\mathrm{x}$-rays emitted by miniature x-ray devices. Phys Med Biol. 1999;44(2):323-333. doi:10.1088/0031-9155/44/2/002

92. Reniers B, Liu D, Rusch T, Verhaegen F. Calculation of relative biological effectiveness of a low-energy electronic brachytherapy source. Phys Med Biol. 2008;53(24):7125-7135. doi:10.1088/0031-9155/53/24/008

93. Rava P, Dvorak T, Markelewicz RJ, et al. A comparison of the biological effective dose of 50-kV electronic brachytherapy with 192Ir high-dose-rate brachytherapy for vaginal cuff irradiation. Brachytherapy. 2012;11(5):402-407. doi:10.1016/j.brachy.2011.08.004

94. Reniers B, Liu D, Rusch T, Verhaegen F. Calculation of relative biological effectiveness of a low-energy electronic brachytherapy source. Phys Med Biol. 2008;53(24):7125-7135. doi:10.1088/0031-9155/53/24/008

95. Zellmer DL, Gillin MT, Wilson JF. Microdosimetric single event spectra of Ytterbium-169 compared with commonly used brachytherapy sources and teletherapy beams. Int J Radiat Oncol Biol Phys. 1992;23(3):627-632. doi:10.1016/0360-3016(92)90021-9 
96. Zellmer DL, Shadley JD, Gillin MT. Comparisons of Measured Biological Response and Predictions from Microdosimetric Data Applicable to Brachytherapy. Radiat Prot Dosimetry. 1994;52(1-4):395-403.

doi:10.1093/oxfordjournals.rpd.a082222

97. Reynoso FJ, Munro III JJ, Cho SH. Technical Note: Monte Carlo calculations of the AAPM TG-43 brachytherapy dosimetry parameters for a new titaniumencapsulated Yb-169 source. J Appl Clin Med Phys. 2017;18(4):193-199. doi:10.1002/acm2.12111

98. Plume CA, Daly SE, Porter A T, Barnett RB, Battista JJ, Battista J. THE RELATIVE BIOLOGICAL EFFECTIVENESS OF YTTERBIUM-169 FOR LOW DOSE RATE IRRADIATION OF CULTURED MAMMALIAN CELLS. Vol 25.; 1993. https://journals-scholarsportalinfo.ezproxy.lib.ryerson.ca/pdf/03603016/v25i0005/835_trbeoyriocmc.xml. Accessed November 14, 2018.

99. Zellmer DL, Gillin MT, Wilson JF. Microdosimetric single event spectra of Ytterbium-169 compared with commonly used brachytherapy sources and teletherapy beams. Int J Radiat Oncol. 1992;23(3):627-632. doi:10.1016/03603016(92)90021-9

100. DENEKAMP, T. WAITES and J. F. FOWLE J. Predicting realistic RBE values for clinically relevant radiotherapy schedules. Int J Radiat Biol. 1997;71(6):681-694. doi:10.1080/095530097143699

101. Lindsay PE, Moiseenko V V, Dyk J Van, Battista JJ. The influence of 
brachytherapy dose heterogeneity on estimates of / for prostate cancer. Phys Med Biol. 2003;48(4):507-522. doi:10.1088/0031-9155/48/4/307

102. Antipas V, Dale RG, Coles IP. A theoretical investigation into the role of tumour radiosensitivity, clonogen repopulation, tumour shrinkage and radionuclide RBE in permanent brachytherapy implants of ${ }^{125} \mathrm{I}$ and ${ }^{103} \mathrm{Pd}$. Phys Med Biol. 2001;46(10):2557-2569. doi:10.1088/0031-9155/46/10/304

103. Armpilia CI, Dale RG, Coles IP, Jones B, Antipas V. The determination of radiobiologically optimized half-lives for radionuclides used in permanent brachytherapy implants. Int J Radiat Oncol. 2003;55(2):378-385. doi:10.1016/S0360-3016(02)04208-6

104. Taylor REP, Rogers DWO. An EGSnrc Monte Carlo-calculated database of TG43 parameters. Med Phys. 2008;35(9):4228-4241. doi:10.1118/1.2965360

105. Karaiskos P, Papagiannis P, Sakelliou L, Anagnostopoulos G, Baltas D. Monte Carlo dosimetry of the selectSeed 125 I interstitial brachytherapy seed. Med Phys. $2001 ; 28(8): 1753-1760$. doi:10.1118/1.1384460

106. Medich DC, Tries MA, Munro JJ. Monte Carlo characterization of an ytterbium169 high dose rate brachytherapy source with analysis of statistical uncertainty. Med Phys. 2005;33(1):163-172. doi:10.1118/1.2147767

107. Taylor REP, Rogers DWO. An EGSnrc Monte Carlo-calculated database of TG43 parameters. 2008. doi:10.1118/1.2965360

108. Agostinelli S, Allison J, Amako K, et al. Geant4-a simulation toolkit. Nucl 
Instruments Methods Phys Res Sect A Accel Spectrometers, Detect Assoc Equip. 2003;506(3):250-303. doi:10.1016/S0168-9002(03)01368-8

109. Sechopoulos I, Rogers DWO, Bazalova-Carter M, et al. RECORDS: improved Reporting of montE CarlO RaDiation transport Studies: Report of the AAPM Research Committee Task Group 268. 2017. doi:10.1002/mp.12702

110. Geant4 Collaboration. Physics Reference Manual.; 2017. https://indico.cern.ch/event/679723/contributions/2792554/attachments/1559217/ 2454299/PhysicsReferenceManual.pdf. Accessed November 16, 2018.

111. Lehnert S, Reniers B, Verhaegen F. Relative biologic effectiveness in terms of tumor response of $125 \mathrm{I}$ implants compared with60Co gamma rays. Int J Radiat Oncol Biol Phys. 2005;63(1):224-229. doi:10.1016/j.ijrobp.2005.05.009

112. Zhuang H-Q, Wang J-J, Liao A-Y, Wang J-D, Zhao Y. The biological effect of $125 \mathrm{I}$ seed continuous low dose rate irradiation in CL187 cells. J Exp Clin Cancer Res. 2009;28(1):12. doi:10.1186/1756-9966-28-12

113. Hering ER. A comparison of the biological effect of ${ }^{125} \mathrm{I}$ and ${ }^{192}$ Ir gamma rays on the roots of Vicia faba using a specially designed applicator. Br J Radiol. 1980;53(627):255-258. doi:10.1259/0007-1285-53-627-255

114. Bianchi C, Botta F, Conte L, Vanoli P, Cerizza L. Biological effective dose evaluation in gynaecological brachytherapy: LDR and HDR treatments, dependence on radiobiological parameters, and treatment optimisation. Radiol Med. 2008;113(7):1068-1078. doi:10.1007/s11547-008-0291-4 
115. Shi C, Guo B, Cheng C-Y, Eng T, Papanikolaou N. Applications of tissue heterogeneity corrections and biologically effective dose volume histograms in assessing the doses for accelerated partial breast irradiation using an electronic brachytherapy source. Phys Med Biol. 2010;55(18):5283-5297. doi:10.1088/0031$9155 / 55 / 18 / 003$

116. Zhao B, Lack D, Spencer B, Miller S, Burmeister J, Liu Q. SU-E-T-649: A Software Tool for Multi-Modality and Multi-Fractionation Biological Composite Plan Evaluation. Med Phys. 2011;38(6Part21):3639-3639. doi:10.1118/1.3612612

117. Guo B, Cheng C, Shi C, Papanikolaou N. MO-G-BRC-03: The Effects of Tissue Inhomogeneities and Biological Effectiveness on the Outcome Prediction of Electronic Brachytherapy for Accelerator Partial Breast Irradiation. Med Phys. 2011;38(6Part27):3736-3736. doi:10.1118/1.3613060

118. Knaup C, Mavroidis P, Stathakis S, Smith M, Swanson G, Papanikolaou N. Evaluation of the effect of prostate volume change on tumor control probability in LDR brachytherapy. J Contemp Brachytherapy. 2011;3:125-130. doi:10.5114/jcb.2011.24818

119. Georg D, Hopfgartner J, Gòra J, et al. Dosimetric Considerations to Determine the Optimal Technique for Localized Prostate Cancer Among External Photon, Proton, or Carbon-Ion Therapy and High-Dose-Rate or Low-Dose-Rate Brachytherapy. Int J Radiat Oncol. 2014;88(3):715-722.

doi:10.1016/j.jijrobp.2013.11.241

120. Villegas F, Bäckström G, Tilly N, Ahnesjö A. Energy deposition clustering as a 
functional radiation quality descriptor for modeling relative biological effectiveness. Med Phys. 2016;43(12):6322-6335. doi:10.1118/1.4966033

121. Maruyama Y, Feola JM, Wierzbicki J, van Nagell JR, Powell D, Yoneda J. Clinical study of relative biological effectiveness for cervical carcinoma treated by ${ }^{252} \mathrm{Cf}$ neutrons and assessed by histological tumour eradication. $\mathrm{Br} J$ Radiol. 1990;63(748):270-277. doi:10.1259/0007-1285-63-748-270

122. Rivard MJ, Melhus CS, Zinkin HD, et al. A Radiobiological Model for the Relative Biological Effectiveness of High-Dose-Rate ${ }^{252}$ Cf Brachytherapy. Radiat Res. 2005;164(3):319-323. doi:10.1667/RR3416.1

123. Antipas V, Dale RG, Coles IP. A theoretical investigation into the role of tumour radiosensitivity, clonogen repopulation, tumour shrinkage and radionuclide RBE in permanent brachytherapy implants of $125 \mathrm{I}$ and $103 \mathrm{Pd}$. 2001;46:2557-2569.

124. M.J. Berger, J.H. Hubbell, S.M. Seltzer, J. Chang, J.S. Coursey, R. Sukumar, D.S. Zucker and KO. XCOM: Photon Cross Sections Database.; 1990. https://www.nist.gov/pml/xcom-photon-cross-sections-database. Accessed November 19, 2018.

125. Berger M, Coursey J, Zucker M, Chang J. Stopping-Power \&amp; Range Tables for Electrons, Protons, and Helium lons | NIST. NIST, Phys Lab. 2017. https://www.nist.gov/pml/stopping-power-range-tables-electrons-protons-andhelium-ions. Accessed December 1, 2017.

126. Hsiao Y, Stewart RD. Monte Carlo simulation of DNA damage induction by $\mathrm{x}$-rays and selected radioisotopes. Phys Med Biol. 2008;53(1):233-244. 
doi:10.1088/0031-9155/53/1/016

127. Cazeca MJ, Medich DC, Munro JJ. Monte Carlo characterization of a new Yb-169 high dose rate source for brachytherapy application. Med Phys. 2010;37(3):11291136. doi:10.1118/1.3301607

128. Beddar AS, Mackie TR, Attix FH. Water-equivalent plastic scintillation detectors for high- energy beam dosimetry: II. Properties and measurements A. Phys Med Biol. 1992;37(10):1883-1900. http://iopscience.iop.org/0031-

9155/37/10/006/pdf/pb921006.pdf.

129. Lambert J, McKenzie DR, Law S, Elsey J, Suchowerska N. A plastic scintillation dosimeter for high dose rate brachytherapy. Phys Med Biol. 2006;51(21):55055516. doi:10.1088/0031-9155/51/21/008

130. Beddar AS. Water equivalent plastic scintillation detectors in radiation therapy. Radiat Prot Dosimetry. 2006;120(1-4):1-6. doi:10.1093/rpd/nci694

131. Beaulieu L, Goulet M, Archambault L, Beddar S. Current status of scintillation dosimetry for megavoltage beams. J Phys Conf Ser. 2013;444(1). doi:10.1088/1742-6596/444/1/012013

132. Archambault L, Polf JC, Beaulieu L, Beddar S. Characterizing the response of miniature scintillation detectors when irradiated with proton beams. Phys Med Biol. 2008;53(7):1865-1876. doi:10.1088/0031-9155/53/7/004

133. Létourneau D, Pouliot J, Roy R. Miniature scintillating detector for small field radiation therapy. Med Phys. 1999;26(12):2555-2561. doi:10.1118/1.598793 
134. Kirov AS, Shrinivas S, Hurlbut C, Dempsey JF, Binns WR, Poblete JL. New water equivalent liquid scintillation solutions for 3D dosimetry. Med Phys. 2000;27(5):1156-1164. doi:10.1118/1.598993

135. Torrisi L. Plastic scintillator investigations for relative dosimetry in proton-therapy. Nucl Instruments Methods Phys Res Sect B Beam Interact with Mater Atoms. 2000;170(3):523-530. doi:10.1016/S0168-583X(00)00237-8

136. Dowkontt et al PF. Towards two-dimensional brachytherapy dosimetry using plastic scintillator: New highly efficient water equivalent plastic scintillator materials. Med Phys. 1999;26(8):1515. http://dx.doi.org/10.1118/1.598647.

137. Frelin AM, Fontbonne JM, Ban G, et al. The DosiMap, a new 2D scintillating dosimeter for IMRT quality assurance: Characterization of two Čerenkov discrimination methods. Med Phys. 2008;35(5):1651-1662. doi:10.1118/1.2897966

138. Reichhart L, Akimov DY, Araújo HM, et al. Quenching factor for low-energy nuclear recoils in a plastic scintillator. Phys Rev C - Nucl Phys. 2012;85(6):1-7. doi:10.1103/PhysRevC.85.065801

139. Group MP. Energy transfer i n organic systems V 111. Quenching of naphthalene fluorescence by biacetyl. 1969;(1).

140. Santiago LM, Bagán H, Tarancón A, Rauret G, Garcia JF. Systematic study of particle quenching in organic scintillators. Nucl Instruments Methods Phys Res Sect A Accel Spectrometers, Detect Assoc Equip. 2013;698:26-36. doi:10.1016/j.nima.2012.09.041 
141. Robertson D, Mirkovic D, Sahoo N, Beddar S. Quenching correction for volumetric scintillation dosimetry of proton beams. Phys Med Biol. 2013;58(2):261-273. doi:10.1088/0031-9155/58/2/261

142. Williamson JF, Dempsey JF, Kirov AS, Monroe JI, Binns WR, Hedtjärn H. Plastic scintillator response to low-energy photons. Phys Med Biol. 1999;44(4):857-871 . doi:10.1088/0031-9155/44/4/004

143. Williamson JF, Dempsey JF, Kirov AS, Monroe JI, Binns WR, Hedtjärn H. Plastic scintillator response to low-energy photons. Phys Med Biol. 1999;44(4):857-871. doi:10.1088/0031-9155/44/4/004

144. Brannen E, Olde GL. The Response of Organic Scintillators to Electron Energy Deposited in Them. Radiat Res. 1962;16(1):1. doi:10.2307/3571123

145. Birks JB. Energy transfer in organic systems VII. Effect of diffusion on fluorescence decay. 1968;1(Forster 1949).

146. Birks JB. Scintillation from organic crystals: Specific flourescecne and relative response to different radiation. Proc Phys Soc A. 1951;64(Hopkins):874-877.

147. Birks B. Energy transfer in organic systems IX. 1969;1704(table 1):513-525.

148. Nyibule S, Tõke J, Henry E, et al. Birks' scaling of the particle light output functions for the EJ 299-33 plastic scintillator. Nucl Instruments Methods Phys Res Sect A Accel Spectrometers, Detect Assoc Equip. 2014;768:141-145. doi:10.1016/j.nima.2014.09.056

149. Hyman M, Ryan JJ. Heavy Elements in Plastic Scintillators. IRE Trans Nucl Sci. 
1958;5(3):87-90. doi:10.1109/TNS2.1958.4315631

150. Tsou KC. Evaluation of Organometallic Compounds for Gamma Detection in Plastic Scintillators. IEEE Trans Nucl Sci. 1965;12(1):28-33. doi:10.1109/TNS.1965.4323493

151. Britvich GI, Vasil'chenko VG, Lapshin VG, Solov'ev AS. New heavy plastic scintillators. Instruments Exp Tech. 2000;43(1):36-39. doi:10.1007/BF02758995

152. Almond PR, Biggs PJ, Coursey BM, et al. AAPM's TG-51 protocol for clinical reference dosimetry of high-energy photon and electron beams. 1999. https://aapm.org/pubs/reports/RPT_67.pdf. Accessed March 27, 2018.

153. Ma CM, Coffey CW, DeWerd LA, et al. AAPM protocol for $40-300 \mathrm{kV} x$-ray beam dosimetry in radiotherapy and radiobiology. Med Phys. 2001;28(6):868-893. doi:10.1118/1.1374247

154. Beddar S, Archambault L, Sahoo N, et al. Exploration of the potential of liquid scintillators for real-time 3D dosimetry of intensity modulated proton beams. Med Phys. 2009;36(5):1736-1743. doi:10.1118/1.3117583

155. Rogers DWO, Bielajew AF. Differences in electron depth-dose curves calculated with EGS and ETRAN and improved energy-range relationships. Med Phys. 1986;13(5):687-694. doi:10.1118/1.595831

156. Dietz-Laursonn E, Hebbeker T, Künsken A, Merschmeyer M, Nieswand S, Niggemann T. GODDeSS: a Geant4 extension for easy modelling of optical detector components. J Instrum. 2017;12(04):P04026-P04026. doi:10.1088/1748- 
0221/12/04/P04026

157. Berger M, Coursey J, Zucker M, Chang J. Stopping-Power and Range Tables for Electrons, Protons, and Helium lons : Physical Reference Data. 1999. https://cds.cern.ch/record/399381?!n=en. Accessed November 9, 2017.

158. Granville DA, Sawakuchi GO. Comparison of linear energy transfer scoring techniques in Monte Carlo simulations of proton beams. Phys Med Biol. 2015;60(14):N283-N291. doi:10.1088/0031-9155/60/14/N283

159. McEwen M, DeWerd L, Ibbott G, et al. Addendum to the AAPM's TG-51 protocol for clinical reference dosimetry of high-energy photon beams. Med Phys. 2014;41(4):041501. doi:10.1118/1.4866223

160. Ma C-M, Coffey CW, Dewerd LA, et al. AAPM protocol for $40-300 \mathrm{kV}$ x-ray beam dosimetry in radiotherapy and radiobiology AAPM protocol for $40-300 \mathrm{kV}$ x-ray beam dosimetry in radiotherapy and radiobiology. 2013;868(2001). doi:10.1118/1.1374247

161. JCGM 100:2008. Evaluation of Measurement Data-Guide to the Expression of Uncertainty in Measurement.; 2008. www.bipm.org. Accessed November 5, 2018.

162. Ma C-M, Coffey CW, Dewerd LA, et al. AAPM protocol for 40-300 kV x-ray beam dosimetry in radiotherapy and radiobiology. 2001. doi:10.1118/1.1374247

163. Eljen Technology. EJ-256 Eljen Datasheet. Sweetwater, TX; 2016. eljentechnology.com/products/plastic-scintillator/ej-256.

164. de Haas JTM, Dorenbos P, van Eijk CWE. Measuring the absolute light yield of 
scintillators. Nucl Instruments Methods Phys Res Sect A Accel Spectrometers, Detect Assoc Equip. 2005;537(1-2):97-100. doi:10.1016/J.NIMA.2004.07.243

165. Boivin J, Beddar S, Bonde C, et al. A systematic characterization of the lowenergy photon response of plastic scintillation detectors. Phys Med Biol. 2016;61(15):5569-5586. doi:10.1088/0031-9155/61/15/5569

166. Sandler SR, Tsou KC. the Scintillation Process in Plastics by Organometallics'. J Phys Chem. 1963;860(1958):300-304. doi:10.1021/j100784a015

167. Gerweck LE, Kozin S V. Relative biological effectiveness of proton beams in clinical therapy. Radiother Oncol. 1999;50(2):135-142. doi:10.1016/S01678140(98)00092-9

168. Karger CP, Peschke P. RBE and related modeling in carbon-ion therapy. Phys Med Biol. 2017;63(1):01TR02. doi:10.1088/1361-6560/aa9102

169. Weyrather WK, Kraft G. RBE of carbon ions: Experimental data and the strategy of RBE calculation for treatment planning. Radiother Oncol. 2004;73:S161-S169. doi:10.1016/S0167-8140(04)80041-0

170. Wolf C, Lafuma J, Masse R, Morin M, Kellerer AM. Neutron RBE for induction of tumors with high lethality in Sprague-Dawley rats. Radiat Res. 2000;154(4):412420. http://www.ncbi.nlm.nih.gov/pubmed/11023605. Accessed June 20, 2019.

171. Athar BS, Paganetti H. Comparison of second cancer risk due to out-of-field doses from 6-MV IMRT and proton therapy based on 6 pediatric patient treatment plans. Radiother Oncol. 2011;98(1):87-92. doi:10.1016/J.RADONC.2010.11.003 
172. Karger CP, Jäkel $\mathrm{O}$, Palmans $\mathrm{H}$, Kanai $\mathrm{T}$. Dosimetry for ion beam radiotherapy. Phys Med Biol. 2010;55(21):193-234. doi:10.1088/0031-9155/55/21/R01

173. Granville DA, Sahoo N, Sawakuchi GO. Calibration of the $\mathrm{Al}_{2} \mathrm{O}_{3}: \mathrm{C}$ optically stimulated luminescence (OSL) signal for linear energy transfer (LET) measurements in therapeutic proton beams. Phys Med Biol. 2014;59(15):42954310. doi:10.1088/0031-9155/59/15/4295

174. Granville DA, Sahoo N. Linear energy transfer dependence of Al2O3:C optically stimulated luminescence detectors exposed to therapeutic proton beams. Radiat Meas. 2014;71:69-73. doi:10.1016/J.RADMEAS.2014.03.026

175. Podgorsak ED, International Atomic Energy Agency. Radiation Oncology Physics: A Handbook for Teachers and Students. International Atomic Energy Agency; 2005. https://inis.iaea.org/search/search.aspx?orig_q=RN:36071456. Accessed July 2, 2019.

176. Guan F, Peeler C, Bronk L, et al. Analysis of the track- and dose-averaged LET and LET spectra in proton therapy using the geant4 Monte Carlo code. Med Phys. 2015;42(11):6234. doi:10.1118/1.4932217

177. Nusrat H, Pang G, Ahmad SB, Keller B, Sarfehnia A. Quantifying the impact of lead doping on plastic scintillator response to radiation. Med Phys. 2019;[In Press]. doi:10.1002/mp.13691

178. Boivin J, Beddar S, Guillemette M, Beaulieu L. Systematic evaluation of photodetector performance for plastic scintillation dosimetry. Med Phys. 2015;42(11):6211-6220. doi:10.1118/1.4931979 
179. Berger M, Hubbel J, Seltzer S, et al. XCOM: Photon Cross Sections Database.; 2010. doi:10.18434/T48G6X

180. Torrisi L. Radiation damage in polyvinyltoluene (PVT). Radiat Phys Chem. 2002;63(1):89-92. doi:10.1016/S0969-806X(01)00487-X

181. Yücel H, Çubukçu, Uyar E, Engin Y. Determination of the energy dependence of the BC-408 plastic scintillation detector in medium energy $\mathrm{x}$-ray beams. Phys Med Biol. 2014;59(22):6749-6758. doi:10.1088/0031-9155/59/22/6749

182. Mizuno T, Kanai Y, Kataoka J, et al. A Monte Carlo method for calculating the energy response of plastic scintillators to polarized photons below $100 \mathrm{keV}$. Nucl Instruments Methods Phys Res Sect A Accel Spectrometers, Detect Assoc Equip. 2009;600(3):609-617. doi:10.1016/j.nima.2008.11.148 


\section{Glossary}

DNA Deoxyribonucleic acid

IMRT Intensity Modulated Radiation Therapy

HDR High dose rate brachytherapy

LDR Low dose rate brachytherapy

LET Linear energy transfer

RBE Relative biological effectiveness

GEANT4 GEometry ANd Tracking 4

FS $\quad$ Field size

VMAT Volumetric modulated arc therapy

LINAC Linear accelerator

MLC Multileaf collimator

$\mathrm{RBE}_{\mathrm{M}} \quad$ Maximum RBE

SBRT Stereotactic body radiotherapy

OAR Organ at risk

EBRT External beam radiotherapy

EBT Electronic brachytherapy

VRT Variance reduction techniques

PTV Planning target volume

PEEK Polyether ethyl ketone

SSD Source to surface distance

PMT Photomultiplier tube 
